The Astrophysical Journal, 616:998-1032, 2004 December 1

(C) 2004. The American Astronomical Society. All rights reserved. Printed in U.S.A.

\title{
ON THE EVOLUTIONARY STATUS OF CLASS I STARS AND HERBIG-HARO ENERGY SOURCES IN TAURUS-AURIGA ${ }^{1}$
}

\author{
Russel J. White and Lynne A. Hillenbrand \\ Department of Astronomy, California Institute of Technology, MS 105-24, Pasadena, CA 91125 \\ Received 2004 May 4; accepted 2004 August 11
}

\begin{abstract}
We present high-resolution $(R \sim 34,000)$ optical (6330-8750 $\AA$ ) spectra obtained with the HIRES spectrograph on the W. M. Keck I telescope of stars in Taurus-Auriga whose circumstellar environment suggests that they are less evolved than optically revealed T Tauri stars. Many of the stars are seen only via scattered light. The sample includes 15 class I stars and all class II stars that power Herbig-Haro flows in this region. For 28 of the 36 stars observed, our measurements are the first high-dispersion optical spectra ever obtained. Photospheric features are observed in all stars with detected continuum, 11 of 15 class I stars (42\% of known Taurus class I stars) and 21 of 21 class II stars; strong emission lines (e.g., $\mathrm{H} \alpha$ ) are detected in the spectra of all stars. These spectra, in combination with previous measurements, are used to search for differences between stars that power HerbigHaro flows and stars that do not and to reassess the evolutionary state of so-called protostars (class I stars) relative to optically revealed T Tauri stars (class II stars). The stellar mass distribution of class I stars is similar to that of class II stars and includes three spectroscopically confirmed class I brown dwarfs. Class I stars (and brown dwarfs) in Taurus are slowly rotating $\left(v \sin i<35 \mathrm{~km} \mathrm{~s}^{-1}\right)$; the angular momentum of a young star appears to dissipate prior to the optically revealed $\mathrm{T}$ Tauri phase. The amount of optical veiling and the inferred mass accretion rates of class I stars are surprisingly indistinguishable from class II stars. Class I stars do not have accretion-dominated luminosities; the accretion luminosity accounts for $\sim 25 \%$ of the bolometric luminosity. The median mass accretion rate of class I and class II stars of K7-M1 spectral type is $4 \times 10^{-8} M_{\odot} \mathrm{yr}^{-1}$, and the median mass outflow rate is $5 \%$ of the mass accretion rate. The large ranges in mass accretion rate $(\sim 2$ orders of magnitude), mass outflow rate $(\sim 3$ orders of magnitude), and the ratio of these quantities ( $\sim 2$ orders of magnitude) represent real dispersions in young accreting stars of similar mass. We confirm previous results that find larger forbidden-line emission associated with class I stars than class II stars. We suggest that this is caused by an orientation bias that allows a more direct view of the somewhat extended forbidden emission line regions than of the obscured stellar photospheres, rather than being caused by larger mass outflow rates. Overall, the similar masses, luminosities, rotation rates, mass accretion rates, mass outflow rates, and millimeter flux densities of class I stars and class II stars are best explained by a scenario in which most class I stars are no longer in the main accretion phase and are much older than traditionally assumed. Similarly, although stars that power Herbig-Haro flows appear to have larger mass outflow rates, their stellar and circumstellar properties are generally indistinguishable from those of similar mass stars that do not power these flows.
\end{abstract}

Subject headings: accretion, accretion disks - circumstellar matter — stars: formation stars: fundamental parameters - stars: low-mass, brown dwarfs — stars: winds, outflows

\section{INTRODUCTION}

Optical spectroscopy both initiated and continues to drive theories of low-mass star formation. Strong $\mathrm{H}$ I and $\mathrm{Ca}$ II emission superposed on a late-type stellar absorption spectrum is one of the original defining characteristics of the T Tauri variable star class (Joy 1945, 1949). Subsequent observations of these emission features have fueled theories for active chromospheres, mass accretion from disks, and mass loss in winds and jets (e.g., Calvet et al. 1984; Lynden-Bell \& Pringle 1974; Decampli 1981). High-resolution optical spectroscopy is still the most accurate tool for characterizing the stellar properties (e.g., $T_{\text {eff }}, \log g, v \sin i$, and $[\mathrm{Fe} / \mathrm{H}]$ ) and investigating mass accretion and stellar jet/wind processes of young stars (JohnsKrull et al. 1999; Stempels \& Piskunov 2003; Hartigan et al.

\footnotetext{
1 The data presented herein were obtained at the W. M. Keck Observatory, which is operated as a scientific partnership among the California Institute of Technology, the University of California, and the National Aeronautics and Space Administration. The Observatory was made possible by the generous financial support of the W. M. Keck Foundation.
}

1995; Muzerolle et al. 1998). For many young accreting stars, high-resolution spectroscopy is often the only tool capable of extracting the stellar photospheric properties from the optically veiled spectrum (e.g., White \& Basri 2003). However, because of high extinction, optical observers have been inhibited from pushing to the earliest stages of low-mass star formation. These objects, often called protostars or class I stars (Lada 1987), consist of a young star embedded within but perhaps beginning to emerge from the collapsing envelope of material from which it is forming. Infall models with infall rates of a few times $10^{-6} M_{\odot} \mathrm{yr}^{-1}$ are consistent with spectral energy distributions (SEDs) and scattered light images of class I stars (e.g., Kenyon et al. 1993a, 1993b; Whitney et al. 1997, assuming a T Taurilike mass and luminosity). This is believed to be the main accretion phase when the majority of stellar mass is acquired. How and how much of the infalling envelope material is transferred onto the central star, as well as the properties of this central star, are largely unknown. Measuring stellar properties and the processes at the star-disk interface generally requires spectroscopic observations at short wavelengths $(\leqslant 2 \mu \mathrm{m})$ 
where these self-embedded stars are the faintest. As a consequence, the current populations of protostars are all assumed to be younger than T Tauri stars because of their less evolved circumstellar environment; there is currently no photospheric evidence that demonstrates that these obscured stars are younger than $\mathrm{T}$ Tauri stars.

The available (but very limited) spectroscopic observations of some embedded stars suggest that they are T Tauri-like (i.e., class II-like) but with heavily veiled spectra and strong emission lines, implying high mass accretion rates and powerful stellar jets (Casali \& Eiroa 1996; Greene \& Lada 1996; Kenyon et al. 1998). The onset of a stellar jet or wind is generally believed to be the mechanism that clears the surrounding envelope, revealing the central star (e.g., Shu et al. 1987). At least $\sim 60 \%$ of all class I objects are associated with Herbig-Haro (HH) objects (Gomez et al. 1997; Kenyon et al. 1998; Reipurth 1999), which are regions of shocked gas believed to be powered by an outflow or jet (Reipurth \& Bally 2001). In comparison, only about $10 \%$ of class II stars appear to power optical outflows, although this subsample has many of the highest accretion rates among $\mathrm{T}$ Tauri stars (e.g., DR Tau, DG Tau; Gullbring et al. 2000). Class II HH energy sources are often associated with spatially extended nebulosity (e.g., HL Tau, FS Tau; Stapelfeldt et al. 1995; Krist et al. 1998). Their resemblance to younger protostars suggests that these stars could be transitioning between the class I and class II stages.

A handful of studies over the last two decades have demonstrated by using low-resolution optical spectra that photospheric features can be seen in the scattered light continua of some class I stars and HH energy sources (Mundt et al. 1985; Cohen et al. 1986; Kenyon et al. 1998). Although the advances in infrared techniques over this same time period led to a widespread shift from optical to infrared observations of the youngest stars (e.g., Greene \& Lada 2000), optical spectroscopy still retains two advantages over near-infrared spectroscopy for characterizing the stellar and accretion properties. First, unlike near-infrared light, which can be dominated by thermal emission from warm dust and gas, optical light is dominated by emission from the photosphere and the hightemperature accretion shocks. It therefore offers the most direct view of the stellar properties and the accretion luminosity. Second, for small dust grains $(\lesssim 1 \mu \mathrm{m})$, optical light scatters more efficiently than near-infrared light. Thus, even if we cannot see the stellar photosphere directly because of very high circumstellar extinction, the cavities commonly seen in the envelopes surrounding class I stars (e.g., Padgett et al. 1999) may allow us to observe the photosphere and inner accretion processes through scattered light. This of course is only feasible in low column density star-forming environments like Taurus-Auriga where the young stars are not deeply embedded within the large-scale molecular cloud. The protostars in Taurus present an opportunity to observe at optical wavelengths young stars at an unprecedentedly early age - potentially up to a factor of 10 younger than more optically revealed T Tauri stars if the statistical ages of embedded stars (Myers et al. 1987; Kenyon et al. 1990) are correct.

In this paper we present a high spectral resolution optical survey of roughly one-half of all objects classified as class I stars (the remaining one-half are currently inaccessible because of faintness) and nearly all objects (including both class I and class II stars) powering HH flows in Taurus. Our goal is to understand how stars evolve from embedded protostars to the optically revealed $\mathrm{T}$ Tauri stars. This requires determining whether class I stars are in fact younger than T Tauri stars and whether they are in the main accretion phase. Our approach is one that we hope will begin to bridge the observational segregation of $\mathrm{T}$ Tauri star and protostellar studies; although both the stellar and circumstellar properties of $\mathrm{T}$ Tauri stars are regularly investigated, the overwhelming majority of protostellar observations are directed toward understanding the circumstellar properties without regard to their stellar properties.

\section{SAMPLE DEFINITION, OBSERVATIONS, AND DATA ANALYSIS}

\subsection{Sample}

The observational goal of this project was to observe a sample of stars in Taurus that are younger than T Tauri stars. Since the ages of young stars are often difficult to determine, especially at the earliest observable stages, we relied on two phenomenological indicators of youth to construct an initial target list. The first was based on measurements of the SED. We identified all stars that are class I-like based on either their midinfrared spectral index (Myers et al. 1987) or their bolometric temperature (Myers \& Ladd 1993). The Infrared Astronomy Satellite (IRAS) provided an unbiased and complete (but fluxand confusion-limited) survey for such objects. The spectral indices, defined as $\alpha=d \log \left(\lambda F_{\lambda}\right) /(d \log \lambda)$, were determined over the wavelength interval $2-25 \mu$ m using the $K-[25]$ colors listed in Kenyon \& Hartmann (1995). We included stars with $\alpha>0$, the generally accepted value used to distinguish class I stars from class II stars (Kenyon \& Hartmann 1995). The bolometric temperatures, defined as the temperature of a blackbody with the same mean frequency as the observed SED, were taken from the compilation of Chen et al. (1995). We included stars with $T_{\text {bol }} \leq 650 \mathrm{~K}$, the value proposed by Chen et al. (1995) to distinguish class I stars from class II stars. This sample consists of 32 stars. We caution that the spectral indices and the bolometric temperatures are in approximately one-half the cases $(57 \%)$ determined from SEDs that include flux contributions from multiple stars. As an extreme example, HH 30 IRS, HL Tau, XZ Tau, and $\mathrm{LkH} \alpha 358$ only have a combined, spatially unresolved $25 \mu \mathrm{m}$ measurement. In these cases the evolutionary classes of the components are assigned that of the integrated system. We note that if the $25 \mu \mathrm{m}$ flux is equally divided among the components and individual indices are calculated using spatially resolved $K$-band magnitudes, these indices generally agree with the system's index (typically \pm 0.2 dex). However, there are remarkable exceptions. If the $25 \mu \mathrm{m}$ flux of $\mathrm{CoKu}$ Tau 1 is divided in this way from its optically bright neighbor Hubble 4 (a weak-lined T Tauri star), its spectral index increases from -0.48 to +0.81 , suggesting that it is a class I star instead of a class II star.

The second indicator of youth we used is the presence of a spatially resolved optical jet. As discussed in the Introduction, stars that power HH flows are almost all either class I stars or nebulous class II stars. Adopting this selection criterion helped to identify a less SED-dependent sample; several apparently very young stars (based on spatially extended circumstellar emission) have insufficient photometric measurements to construct a SED (e.g., CoKu Tau 1, HV Tau C) and would have been excluded otherwise. Specifically, we included all stars that are "Suspected Sources" of HH jets/flows in Taurus-Auriga as listed in the General Catalogue of HH Objects (Reipurth 1999). We also included the young star ZZ Tau IRS, which is the suspected energy source of HH 393 (Gomez et al. 1997) and has never been observed spectroscopically. We refer to stars that power an HH flow as $\mathrm{HH}$ stars and those that do not as 
non-HH stars. This second selection criterion added 12 additional stars, for a total sample of 44 stars.

In light of the circumstellar selection criteria used to identify this sample, we refer to stars in this sample as environmentally young. We distinguish a strict subsample of class I stars, by requiring that both $\alpha>0.0$ and $70 \mathrm{~K}<T_{\text {bol }}<650 \mathrm{~K}$ (if only one of these SED measurements is available, the class classification is based solely on that quantity). Stars with $T_{\text {bol }}<70 \mathrm{~K}$ are considered class 0 stars. Following this criterion, 26 of the 44 environmentally young stars are class I stars, and one is a class 0 star (RAS 04368+2557). The remaining 17 stars are classified as class II stars.

This combined sample of 44 stars is listed in Table 1 and is presumed to include the youngest stars in Taurus; the list does not include stars not detected at $2 \mu \mathrm{m}$ (e.g., IRAM 04191+ 1522; starless cores, Motte \& André 2001; Onishi et al. 2002). The components of binary systems (e.g., Ghez et al. 1993; Leinert et al. 1993; Ducheñe et al. 2004) are indicated with the suffix A or B, while the spatially unresolved multiples are indicated with the suffix AB or ABC. Stars newly observed in this study are distinguished (new) in the third column from stars for which we use previous high-dispersion measurements (prev). Coordinates from the 2MASS database are given and in most cases are a substantial improvement on the available astrometry, especially for stars within dark cloud cores. We also list the $2-25 \mu \mathrm{m}$ spectral indices, bolometric temperatures from Chen et al. (1995), our evolutionary classification (I or II), whether the source powers a spatially resolved optical jet (Gomez et al. 1997; Reipurth 1999), whether the star is visible in the POSS-II Red plates (inspection by eye), $I_{c}$ magnitudes and references, and 2MASS $J$-band magnitudes. Thirty-one of the 44 environmentally young stars power an optical jet; 15 of the 27 class I/0 stars have optical jets. Twenty-eight of these 44 stars are visible on the red POSS-II plates and thus emit some observable light shortward of $1 \mu \mathrm{m}$; the 16 stars not visible consist of 15 class I stars and one (borderline) class II star, IRAS 04154+2823.

Figure 1 shows the distributions of $\alpha$ and $T_{\mathrm{bol}}$ for the stars spectroscopically observed here $(\S 2.3)$ and for the larger sample of Taurus T Tauri stars in Kenyon \& Hartmann (1995) that have both measurements available. For illustrative purposes, the three class I stars without $T_{\text {bol }}$ estimates (IRAS $04260+$ 2642, IRAS 04325+2402, and Haro 6-28) are plotted assuming a value of $500 \mathrm{~K}$, and the one class II star without a $T_{\text {bol }}$ estimate $(\mathrm{CoKu}$ Tau 1$)$ is plotted assuming a value of $2000 \mathrm{~K}$.

\section{2. $I_{c}$-band Imaging, Reduction, and Photometry}

Using the W. M. Keck II telescope and the Low Resolution Imaging Spectrograph (LRIS; Oke et al. 1995) in its imaging mode, we obtained $I_{c}$-band images of 17 of the 44 environmentally young stars (mostly class I stars) listed in Table 1 on 1998 October 30-31 and 1999 December 13. In all cases, the integration time was set to $300 \mathrm{~s}$, which yields a sky count level of greater than one-half full-well. The images are $6 \times 8 \mathrm{arcmin}^{2}$ in size and were processed by subtracting a median-averaged set of bias frames and dividing by a median-filtered set of flatfield frames constructed from observation of the twilight sky. Equatorial "selected area" standards (Landolt 1992) were observed for photometric calibration, assuming typical Mauna Kea extinction; all images were obtained at an air mass of less than 1.1. Magnitudes were extracted using an aperture 6.3 in diameter and a sky annulus extending from $26^{\prime \prime}$ to $30^{\prime \prime}$. These values are listed in Table 1. Errors are less than 0.1 mag unless marked with a colon. The measurement errors for stars listed with a colon are larger and more difficult to quantify because their emission is primarily spatially extended.

\subsection{High-Resolution Optical Spectroscopy}

The W. M. Keck I telescope and High-Resolution Echelle Spectrometer (HIRES; Vogt et al. 1994) were used by us to conduct observations on three separate observing runs: 1999 December 5-6, 2002 December 13, and 2003 February 17-18. Variable and sometimes heavy cirrus conditions dominated the 1999 December run, while the other two runs had apparently photometric conditions. HIRES was used with the red collimator and the RG-610 filter. The D1 decker $\left(11^{\prime \prime} 15 \times 14^{\prime \prime}\right)$ was used, which has a projected slit width of 4 pixels, yielding a resolving power of $\sim 34,000$. The cross-disperser and echelle angles were set (at approximately 1.41 and -0.28 , respectively) to achieve a wavelength coverage of $\sim 6330-8750 \AA$, spanning 16 orders with wavelength gaps between the orders. This wavelength range includes several regions with temperaturesensitive photospheric features and many of the strongest permitted emission lines ( $\mathrm{H} \alpha, \mathrm{O}$ I 8446, Ca II 8498, and Ca II 8662) and forbidden emission lines ([O I] 6364, [N II] 6583, [S II] 6716 , and [S II] 6731) seen in T Tauri star spectra. Calibration frames obtained included those of an internal quartz lamp for flat-fielding and a ThAr lamp for wavelength calibration.

From the environmentally young star sample listed in Table 1, observational priority was given to targets that showed optical emission in the POSS-II Red plates (28 of 44) and for which no previous high-resolution spectra were available. We observed 23 of the 28 optically visible stars. The remaining five stars have been observed previously with either high-dispersion optical spectroscopy (CW Tau, GK Tau, DO Tau, and UY Aur; Hartigan et al. 1995) or spatially resolved medium-dispersion optical spectroscopy (XZ Tau A and B; Hartigan \& Kenyon 2003). We include the available results of these previous measurements in our analysis and discussion ( $\S \S 3$ and 4). Finally, we observed three of the 16 environmentally young stars that are not visible on POSS-II plates but are seen in our LRIS images (IRAS 04154+ 2823 , IRAS $04295+2251$, and IRAS 04489+3042), yielding a total of 26 of the 44 stars listed in Table 1, 31 including previous spectroscopy.

In addition to these primary targets, we obtained spectra of six additional Taurus T Tauri star systems. IRAS 04278+2253 A and B, Haro 6-13, and IRAS 04303+2240 all have bolometric temperatures less than $1000 \mathrm{~K}$ and have never been observed at high dispersion. HK Tau A and B have been classified as transitional class I/II stars (Kenyon \& Hartmann 1995); HK Tau B is also an edge-on disk system (Stapelfeldt et al. 1998; Koresko 1998) and has never been observed optically at high dispersion. We observed this pair of stars, even though the system's $\alpha$ and $T_{\text {bol }}$ do not corroborate the claim that it is a transitional class I/II star $\left(\alpha=-0.51, T_{\text {bol }}=2148 \mathrm{~K}\right)$. The lightly veiled T Tauri stars DN Tau and V836 Tau were observed as well. The properties of these six star systems are also listed in Table 1, separately. They are included in the analysis but distinguished from the environmentally young star sample.

The spectroscopic observations spatially resolved the components of the binary systems MHO 1/2 IRAS $04278+2253$, HK Tau, and RW Aur. For the HV Tau system, only a spectrum of HV Tau C was obtained. In the case of L1551 IRS 5, we obtained spectra of the pointlike optically visible object at the position of IRS 5, at the position of the HH object $\sim 12^{\prime \prime}$ east of IRS 5, and at a position centered on the brightest portion of the optical reflection nebula that is 2.5 directly west of IRS 5 (see Stocke et al. 1988). 
TABLE 1

A Sample of Young Stars in Taurus-Auriga

\begin{tabular}{|c|c|c|c|c|c|c|c|c|c|c|c|c|}
\hline $\begin{array}{l}\text { Common } \\
\text { Name }\end{array}$ & $\begin{array}{l}\text { Associated } \\
\text { IRAS Source }\end{array}$ & Obs? $^{\mathrm{a}}$ & R.A. $(2000)^{\text {b }}$ & Decl. $(2000)^{\mathrm{b}}$ & $\begin{array}{l}\text { Spectral } \\
\text { Index }^{c}\end{array}$ & $\begin{array}{l}T_{\mathrm{bol}}{ }^{\mathrm{c}} \\
(\mathrm{K})\end{array}$ & $\begin{array}{l}\text { Evolutionary } \\
\text { Class }^{\mathrm{d}}\end{array}$ & HH Source? ${ }^{\mathrm{e}}$ & $\begin{array}{c}\text { Visible } \\
\text { in DSS? }\end{array}$ & $\begin{array}{c}I_{\mathrm{C}} \\
(\mathrm{mag})\end{array}$ & $\begin{array}{c}J^{\mathrm{g}} \\
(\mathrm{mag})\end{array}$ & $I_{\mathrm{C}}$ Reference \\
\hline \multicolumn{13}{|c|}{ Environmentally Young Stars in Taurus-Auriga } \\
\hline *.. & $04016+2610$ & new & 040443.27 & +261853.6 & +1.01 & 238 & I' & Yes & Yes & 17.94 & 12.72 & 1 \\
\hline * & $04108+2803 \mathrm{~B}$ & $\ldots$ & 041354.72 & +28 1132.9 & $(+0.61)$ & (205) & I' & $\ldots$ & No & 22.1: & 16.48 & 2 \\
\hline CW Tau .................................. & $04112+2803$ & prev & 041417.00 & +28 1057.8 & -0.44 & 3207 & II & Yes & Yes & 11.42 & 9.54 & 1 \\
\hline MHO $2 / 1^{\dagger} \ldots \ldots \ldots \ldots \ldots \ldots$ & $04113+2758 \mathrm{AB}$ & new & 041426.40 & +280559.7 & $(+0.10)$ & (606) & I & $\ldots$ & Yes & $\ldots$ & 11.14 & 2 \\
\hline * & $04154+2823$ & new & 041832.03 & +283115.4 & -0.03 & 650 & II & $\ldots$ & No & $\ldots$ & 15.19 & 2 \\
\hline CoKu Tau 1 AB ............. & $04157+2813$ & new & 041851.48 & +282026.5 & $(-0.48)$ & $\ldots$ & II & Yes & Yes & $\ldots$ & 12.87 & 2 \\
\hline * & $04158+2805$ & new & 041858.14 & +281223.5 & +0.71 & 528 & I' & $\ldots$ & Yes & $\ldots$ & 13.78 & 2 \\
\hline 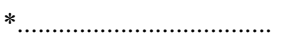 & $04166+2706$ & $\ldots$ & 041942.5 & +271340 & +0.48 & 139 & I & Yes & No & $>24:$ & & 2 \\
\hline 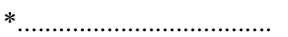 & $04169+2702$ & $\ldots$ & 041958.45 & +270957.1 & +1.09 & 170 & I & Yes & No & 20.1: & 17.19 & 2 \\
\hline 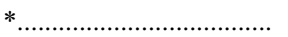 & $04181+2655$ & $\ldots$ & 042107.95 & +2702 20.4 & +0.54 & 278 & I & Yes & No & 22.5: & 13.86 & 2 \\
\hline * & $04181+2654 \mathrm{~A}$ & $\ldots$ & 042111.47 & +270109.4 & $(+0.09)$ & (346) & I & $\ldots$ & No & 19.9: & 16.22 & 2 \\
\hline 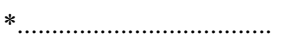 & $04181+2654 \mathrm{~B}$ & $\ldots$ & 042110.39 & +270137.3 & $(+0.09)$ & (306) & I & $\ldots$ & No & $>24:$ & 18.89 & 2 \\
\hline 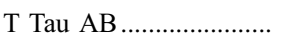 & $04190+1924$ & new & 042159.43 & +193206.4 & $(-0.15)$ & $(3452 / 501)$ & II & Yes & Yes & 8.50 & 7.24 & 1 \\
\hline Haro 6-5 В ....................... & $04189+2650$ & new & 042200.70 & +265732.5 & $(-0.15)$ & $(5948)$ & II & Yes & Yes & $\ldots$ & 15.08 & 2 \\
\hline * & $04239+2436$ & $\ldots$ & 042656.30 & +244335.3 & $(+1.13)$ & $(236)$ & I & Yes & No & 19.4: & 15.75 & 2 \\
\hline DG Tau B ......................... & $04240+2559$ & new & 042702.67 & +260530.4 & $(+0.18)$ & (1440) & II & Yes & Yes & $\ldots$ & 14.72 & 2 \\
\hline 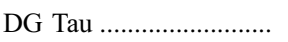 & $04240+2559$ & new & 042704.70 & +260616.3 & $(+0.18)$ & (1440) & II & Yes & Yes & 10.54 & 8.69 & 1 \\
\hline HH31 IRS 2 AB ............ & $04248+2612$ & new & 042757.33 & +261918.1 & $(+0.47)$ & (334) & I & Yes & Yes & 16.9: & 11.62 & 2 \\
\hline 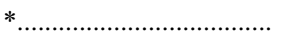 & $04260+2642$ & new & 042904.99 & +264907.3 & +0.24 & & I' & $\ldots$ & Yes & $\ldots$ & 14.68 & 2 \\
\hline 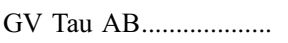 & $04263+2426$ & new & 042923.73 & +243300.3 & $(+0.79)$ & (409) & I & Yes & Yes & 12.80 & 11.54 & 1 \\
\hline HH 414 IRS AB ............ & $04264+2433$ & new & 042930.08 & +243955.1 & $(+0.97)$ & $(252)$ & I' & Yes & Yes & 18.5: & 13.73 & 2 \\
\hline ZZ Tau IRS ....................... & $04278+2435$ & new & 043051.71 & +244147.5 & $(-1.46)$ & (2048) & II & Yes & Yes & $\ldots$ & 12.84 & 2 \\
\hline L1551 IRS 5 & $04287+1801$ & new & 043134.08 & +180804.9 & +1.76 & 97 & I & Yes & Yes & 18.6: & 13.71 & 2 \\
\hline HH 30 IRS ............................ & $04287+1807$ & new & 043137.47 & +181224.5 & $(+0.35)$ & (913) & II & Yes & Yes & 17.3 & 15.18 & 4 \\
\hline HL Tau .............................. & $04287+1807$ & new & 043138.44 & +181357.7 & $(+0.35)$ & $(576)$ & I' & Yes & Yes & 12.56 & 10.62 & 1 \\
\hline 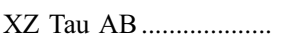 & $04287+1807$ & prev & 043140.07 & +181357.2 & $(+0.35)$ & (1250) & II & Yes & Yes & 12.00 & 9.36 & 1 \\
\hline L1551 NE ........................... & $04287+1801$ & $\ldots$ & 043144.45 & +180831.5 & +1.69 & 75 & I & Yes & No & $\ldots$ & 16.61 & 2 \\
\hline 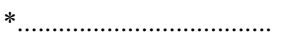 & $04295+2251$ & new & 043232.05 & +225726.7 & +0.20 & 447 & I' & $\ldots$ & No & 20.0: & 14.89 & 2 \\
\hline 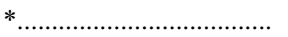 & $04302+2247$ & new & 043316.50 & +225320.4 & +0.13 & 202 & I' & Yes & Yes & $\ldots$ & 14.68 & 2 \\
\hline \multirow[t]{2}{*}{ GK Tau .............................. } & $04305+2414$ & prev & 043334.56 & +242105.9 & $(-0.67)$ & $(2667)$ & II & Yes & Yes & 10.62 & 9.05 & 1 \\
\hline & $04325+2402 \mathrm{ABC}$ & 1 & 043535.39 & +240819.4 & $(+0.78)$ & & I & $\ldots$ & No & 21.5: & 17.13 & 2 \\
\hline Haro 6-28 AB .................. & $04328+2248$ & new & 043556.84 & +225436.0 & $(+0.38)$ & & I: & $\ldots$ & Yes & $\ldots$ & 11.14 & 2 \\
\hline DO Tau ............................ & $04353+2604$ & prev & 043828.58 & +261049.4 & $(-0.51)$ & $(2125)$ & II & Yes & Yes & 11.17 & 9.47 & 1 \\
\hline HV Tau C ........................ & $04353+2604$ & new & 043835.48 & +261041.5 & $(-0.51)$ & $(2125)$ & II & Yes & Yes & 14.93 & 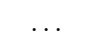 & 3 \\
\hline 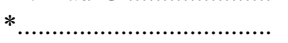 & $04361+2547 \mathrm{AB}$ & $\ldots$ & 043913.89 & +255320.9 & $(+1.54)$ & (144) & I & $\ldots$ & No & 22.3: & 16.44 & 2 \\
\hline 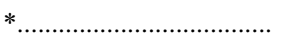 & $04365+2535$ & $\ldots$ & 043935.19 & +254144.7 & +1.26 & 172 & I & $\ldots$ & No & 23.5: & 16.91 & 2 \\
\hline 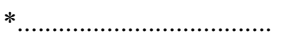 & $04368+2557$ & $\ldots$ & 043953.8 & +260306 & +0.74 & 59 & 0 & Yes & No & 19.0: & $\ldots$ & 2 \\
\hline IC $2087 \ldots \ldots \ldots \ldots \ldots$ & $04369+2539$ & new & 043955.75 & +254502.0 & -0.57 & 860 & II & Yes & Yes & $\ldots$ & 10.66 & 2 \\
\hline * & $04381+2540$ & $\ldots$ & 044112.68 & +254635.4 & +1.29 & 139 & I & Yes & No & 21.1: & 17.15 & 2 \\
\hline 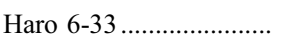 & $04385+2550$ & new & 044138.82 & +255626.8 & +0.17 & 636 & I' & Yes & Yes & 15.58 & 11.85 & 1 \\
\hline DP Tau ......................... & $04395+2509$ & new & 044237.70 & +251537.5 & -0.44 & 1991 & II & Yes & Yes & 11.95 & 11.00 & 1 \\
\hline UY Aur AB........................ & $04486+3042$ & prev & 045147.38 & +304713.5 & $(-0.29)$ & $(2120)$ & II & Yes & Yes & 10.83 & 9.13 & 1 \\
\hline 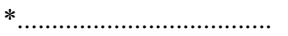 & $04489+3042$ & new & 045206.68 & +304717.5 & +0.29 & 399 & I' & 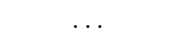 & No & 20.3: & 14.43 & 2 \\
\hline 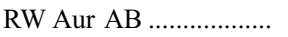 & $05046+3020$ & new & 050749.54 & +302405.1 & $(-0.56)$ & $(4478)$ & II & Yes & Yes & 9.34 & 8.38 & 1 \\
\hline
\end{tabular}


TABLE 1 -Continued

\begin{tabular}{|c|c|c|c|c|c|c|c|c|c|c|c|c|}
\hline $\begin{array}{l}\text { Common } \\
\text { Name }\end{array}$ & $\begin{array}{l}\text { Associated } \\
\text { IRAS Source }\end{array}$ & Obs? ${ }^{\mathrm{a}}$ & R.A. $(2000)^{\mathrm{b}}$ & Decl. $(2000)^{\mathrm{b}}$ & $\begin{array}{l}\text { Spectral } \\
\text { Index }^{c}\end{array}$ & $\begin{array}{l}T_{\text {bol }}{ }^{\mathrm{c}} \\
(\mathrm{K})\end{array}$ & $\begin{array}{l}\text { Evolutionary } \\
\text { Class }^{\mathrm{d}}\end{array}$ & HH Source? ${ }^{\mathrm{e}}$ & $\begin{array}{c}\text { Visible } \\
\text { in DSS? }^{\mathrm{f}}\end{array}$ & $\begin{array}{c}I_{\mathrm{C}} \\
(\mathrm{mag})\end{array}$ & $\begin{array}{c}J^{\mathrm{g}} \\
(\mathrm{mag})\end{array}$ & $I_{\mathrm{C}}$ Reference \\
\hline \multicolumn{13}{|c|}{ Additional T Tauri Stars in Taurus-Auriga } \\
\hline 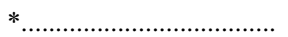 & $04278+2253$ & new & 043050.28 & +230008.8 & $(-0.34)$ & $(856)$ & II & $\ldots$ & Yes & $\ldots$ & 8.78 & 2 \\
\hline HK Tau …………….... & $04288+2417$ & new & 043150.57 & +242418.1 & $(-0.51)$ & $(2148)$ & II & $\ldots$ & Yes & 12.37 & 10.45 & 1 \\
\hline Haro $6-13$............... & $04292+2422$ & new & 043215.41 & +242859.7 & -0.20 & 910 & II & $\ldots$ & Yes & $\ldots$ & 11.24 & 2 \\
\hline * & $04303+2240$ & new & 043319.07 & +224634.2 & -0.35 & 886 & II & $\ldots$ & Yes & 15.73 & 11.10 & 1 \\
\hline 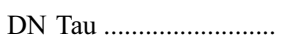 & $04324+2408$ & new & 043527.37 & +241458.9 & -0.84 & 2890 & II & No & Yes & 10.49 & 9.14 & 1 \\
\hline 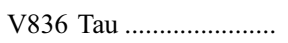 & $\ldots$ & new & 050306.60 & +252319.7 & -0.93 & 3462 & II & No & Yes & 11.19 & 9.91 & 1 \\
\hline
\end{tabular}

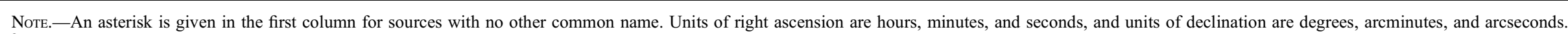

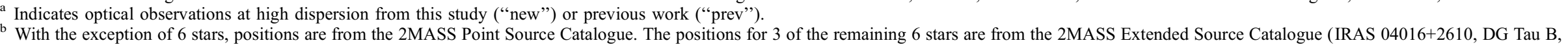

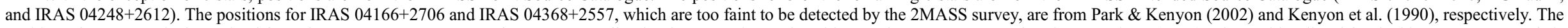

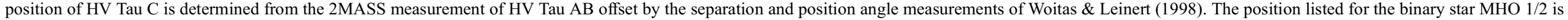
that of the optically brighter star MHO 2.

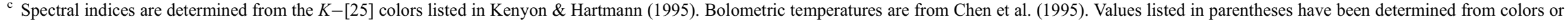
SEDs that include contributions from more than 1 star.

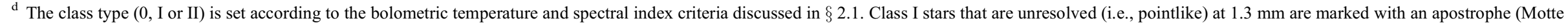
\& André 2001); Haro 6-28, which is marked with a colon, has no spatial millimeter information.

${ }^{\mathrm{e}}$ Indicates whether the star powers a Herbig-Haro flow (Gomez et al. 1997; Reipurth 1999).

${ }^{\mathrm{f}}$ Indicates whether the object is visible in the Second Generation POSS-II Red plates.

g $J$ magnitudes are from the 2MASS Point Source Catalogue except for IRAS 04016+2610, DG Tau B, and IRAS 04248+2612, which are from the 2MASS Extended Source Catalogue.

ReFerences.-(1) Kenyon \& Hartmann 1995; (2) this work; (3) Magazzú \& Martín 1994; (4) Mundt \& Fried 1983. 


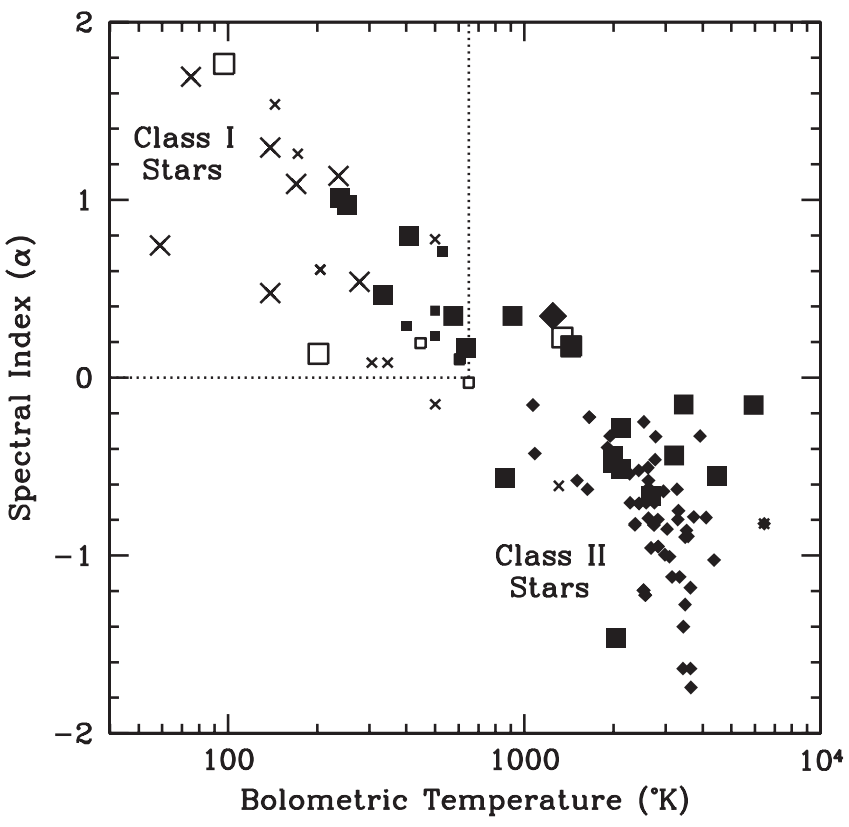

FIG. 1.- Spectral index vs. bolometric temperature for stars in Taurus. A total of 44 stars have either $T_{\text {bol }}<650 \mathrm{~K}$ or $\alpha>0.0$ or power an HH flow. We consider these to be environmentally young stars (Table 1). The subset of class I stars have both $T_{\text {bol }}<650 \mathrm{~K}$ and $\alpha>0.0$ (when both values are available; see text). Large symbols indicate stars that power $\mathrm{HH}$ flows while small symbols represent stars that do not. Diamonds indicate stars that have been observed spectroscopically previously (Basri \& Batalha 1990; Hartigan et al. 1995; White \& Basri 2003) and squares indicate stars observed in this study, 15 of which are within the class I regime. Of the 36 stars observed here, six have no measurable continuum (unfilled squares; only five are visibly plotted, since MHO-1 and MHO-2 overlap), while 30 (3 of which are binary star components with overlapping positions) show a stellar continuum (filled squares) that we use to study the stellar and accretion properties. The X's indicate stars that have never been observed at high dispersion.

For 28 of the 36 stars observed (in 32 systems), these measurements are the first high-dispersion optical spectra obtained. Exposure times and epochs of observations are listed in Table 2. To assist in the spectroscopic analysis, we observed four slowly rotating weak-lined T Tauri stars in the TW Hydrae association (TWA 8 A and B and TWA 9 A and B) and numerous dwarf and giant spectral type standards, nine of which have accurately known radial velocities (Nidever et al. 2002). The observed spectroscopic standards were chosen to span a range of $\mathrm{G}, \mathrm{K}$, and $\mathrm{M}$ spectral types at both dwarf and giant surface gravities.

\subsection{Spectroscopic Reduction}

The HIRES data were reduced using the facility makee reduction script written by T. Barlow. The reductions included bias subtraction, flat-fielding, spectral extraction, sky subtraction, wavelength calibration, and heliocentric radial velocity corrections. The projected spectra from target stars have typical full widths at half-maximum of $1^{\prime \prime}-2^{\prime \prime}$, although five stars (IRAS 04016+2610, IRAS 04302+2247, L1551 IRS 5, DG Tau B, and Haro 6-5 B) have much broader profiles because of spatially extended emission. In these cases, a broader extraction aperture was used to include this extended emission; the sky level was determined outside these apertures.

The two binary stars RW Aur and IRAS 04278+2253 were observed with the components aligned along the slit. The spectra of the components were extracted with a choice of apertures that minimize contamination from the companion. In the case of RW Aur, in which the secondary is separated by only 1 1"4 from the primary and is nearly 10 times fainter, the extracted spectrum of the secondary star is still contaminated by that of the primary; the secondary's spectrum showed a broad but very distinct low-level component to the $\mathrm{Ca}$ II emission lines $(\lambda 8498, \lambda 8662)$, as well as very weak O I circumstellar absorption, both of which we attribute to contamination by RW Aur A. These artifact features disappear cleanly if a spectrum of RW Aur A, scaled to $20 \% \pm 5 \%$ of RW Aur B's continuum level, is subtracted from the spectrum of RW Aur B. Our final reduced spectrum of RW Aur B has this $20 \%$ subtraction.

Figures 2 and 3 show portions of the HIRES spectra for the 28 stars with a continuum signal-to-noise ratio $(\mathrm{S} / \mathrm{N})$ greater than 2 . Table 2 lists the continuum $\mathrm{S} / \mathrm{N}$ per pixel at 6700 and $8400 \AA$ for all observations. For the six stars with $\mathrm{S} / \mathrm{N}<2$ (two class II stars: DG Tau B and IRAS 04154+2823, and four class I stars: MHO 1, L1551 IRS 5, IRAS 04295+2251, and IRAS $04302+2247$ ) the continuum detected (if real) is insufficient to measure any photospheric lines. These six stars are excluded from our photospheric analyses. Li I (6708 $⿱$ ) is detected in the spectra of 24 of the 28 stars with a genuine continuum. $\mathrm{H} \alpha$ emission is detected in all 36 stars observed.

\section{ANALYSIS}

Figure 1 illustrates how this survey has extended the sample of stars with spectroscopic detections of the photosphere well into the class I regime. Of the 26 class I stars in Taurus (Table 1), 11 now have spectroscopic measurements of their photospheres and 15 have optical emission-line measurements. In addition to the remaining 15 class I stars with no spectroscopic detection of their photosphere, there are seven class II stars from Kenyon \& Hartmann (1995) with no spectroscopically measured photospheres: IRAS 04370+2559, IRAS 04414+2506, IRAS 04301+ 2608, FZ Tau, FT Tau, IRAS 04200+2759, and T Tau South. Note that only three of these class II stars have both $\alpha$ and $T_{\text {bol }}$ measurements and thus are shown in Figure 1.

Though not complete, the considerable expansion of the available spectroscopy toward environmentally younger stars enables for the first time an investigation of the stellar and circumstellar properties between evolutionary classes. We wish to accomplish this without relying too strongly on any one evolutionary diagnostic ( $T_{\mathrm{bol}}, \alpha, \mathrm{HH}$ properties), which may be flawed and/or have unaccounted for biases. Therefore, we take the approach of comparing all measured and inferred properties with both the bolometric temperatures and spectral indices (when available), and distinguishing $\mathrm{HH}$ stars from non-HH stars. Possible differences between the properties of class I stars and class II stars (defined by $T_{\text {bol }}$ and $\alpha$ criteria; $\S 2.1$ ) and $\mathrm{HH}$ stars and non-HH stars are investigated by comparing median values and by conducting K-S tests on these distributions (Press et al. 1997). K-S probabilities are reported in most cases; we consider distributions to be different if the $\mathrm{K}-\mathrm{S}$ probability is less than 0.056 (i.e., $2 \sigma$ difference).

To increase the significance of these comparisons, we identify and include an additional sample of class II T Tauri stars. This sample includes the six additional Taurus $\mathrm{T}$ Tauri stars observed here (all optically veiled) and the 26 optically veiled (at $\sim 6000 \AA$ ) Taurus T Tauri stars from the high spectral resolution studies of Basri \& Batalha (1990), Hartigan et al. (1995), and White \& Basri (2003). Our comparisons thus include only stars that either are environmentally young (Table 1) or are classical $\mathrm{T}$ Tauri stars defined by their optically veiled 
TABLE 2

Observational Summary and Measured Photospheric Properties

\begin{tabular}{|c|c|c|c|c|c|c|c|c|c|c|}
\hline Star & Obs. Date & $\begin{array}{c}\text { Exposure } \\
\text { Time } \\
\text { (minutes) }\end{array}$ & $\mathrm{S} / \mathrm{N}_{6700}$ & $\mathrm{~S} / \mathrm{N}_{8450}$ & $\begin{array}{c}\mathrm{EW}[\mathrm{Li} \mathrm{I}] \\
(\AA)\end{array}$ & $\begin{array}{c}\text { Radial } \\
\text { Velocity } \\
\left(\mathrm{km} \mathrm{s}^{-1}\right)\end{array}$ & $\begin{array}{c}v \sin i \\
\left(\mathrm{~km} \mathrm{~s}^{-1}\right)\end{array}$ & $\begin{array}{l}\text { Spectral } \\
\text { Type }\end{array}$ & $r_{6500}$ & $r_{8400}$ \\
\hline \multicolumn{11}{|c|}{ Environmentally Young Stars in Taurus-Auriga } \\
\hline IRAS $04016+2610 \ldots \ldots \ldots \ldots \ldots \ldots \ldots$ & 1999 Dec 5, 6 & 215 & 1.6 & 2.1 & $\leq 0.5$ & $19 \pm 7:$ & $\leq 15:$ & $\mathrm{K}:$ & $\leq 0.3:$ & $\leq 0.37$ \\
\hline 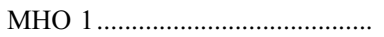 & 2003 Feb 17 & 10 & 0.1 & 1.3 & $\leq 4.2$ & $\ldots$ & $\ldots$ & $\ldots$ & $\ldots$ & $\ldots$ \\
\hline MHO 2 & 2003 Feb 17 & 30 & 5.0 & 18 & $0.39 \pm 0.06$ & $14.8 \pm 3.0$ & $21.5 \pm 4.2$ & $\mathrm{M} 3.5 \pm 1$ & $<0.24$ & $<0.05$ \\
\hline IRAS $04154+2823 \ldots \ldots \ldots \ldots \ldots \ldots \ldots$ & 2003 Feb 17 & 60 & 0.2 & 0.9 & $\leq 1.4$ & $\ldots$ & $\ldots$ & $\ldots$ & $\ldots$ & $\ldots$ \\
\hline 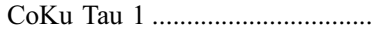 & 2002 Dec 13 & 30 & 8.5 & 15 & $0.49 \pm 0.03$ & $15.0 \pm 0.8$ & $15.3 \pm 1.7$ & $\mathrm{~K} 7 \pm 1+\mathrm{M}^{\mathrm{a}}$ & $0.38 \pm 0.18$ & $\leq 0.19$ \\
\hline 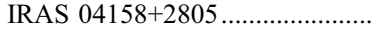 & 2002 Dec 13 & 30 & 4.0 & 10 & $0.21 \pm 0.07$ & $18.1 \pm 5.4$ & $23.1 \pm 4.5$ & $\mathrm{M} 6 \pm 1$ & $\leq 1.0$ & $0.58 \pm 0.30$ \\
\hline Т Tau & 2003 Feb 18 & 1.5 & 144 & 119 & $0.36 \pm 0.02$ & $20.2 \pm 0.4$ & $20.9 \pm 1.0$ & $\mathrm{~K} 0 \pm 2$ & $\leq 0.20$ & $\leq 0.19$ \\
\hline Haro 6-5 В & 2002 Dec 13 & 20 & 7.0 & 5.1 & $0.45 \pm 0.04$ & $18.6 \pm 3.6$ & $20.7 \pm 6.4$ & $\mathrm{~K} 5 \pm 2$ & $1.0 \pm 0.2$ & $1.4 \pm 1.2$ \\
\hline 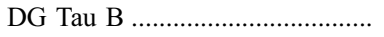 & 2002 Dec 13 & 30 & 0.6 & 0.4 & $\leq 0.5$ & $\ldots$ & $\ldots$ & $\ldots$ & $\ldots$ & $\ldots$ \\
\hline 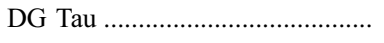 & 1999 Dec 6 & 15 & 59 & 65 & $0.24 \pm 0.02$ & $19.3 \pm 2.7$ & $28.6 \pm 5.1$ & $\mathrm{~K} 3 \pm 2$ & $2.1 \pm 0.5$ & $1.8 \pm 0.5$ \\
\hline IRAS $04248+2612 \ldots \ldots \ldots \ldots \ldots \ldots$ & 1999 Dec 6 & 180 & 1.3 & 4.4 & $\leq 0.2$ & $14.4 \pm 2.5$ & $16.4 \pm 4.2$ & $\mathrm{M} 5.5 \pm 1$ & 1.4: & $0.60 \pm 0.21$ \\
\hline IRAS $04260+2642 \ldots \ldots \ldots \ldots \ldots \ldots$ & 2002 Dec 13 & 60 & 5.3 & 8.2 & $0.43 \pm 0.05$ & $22.3 \pm 3.6$ & $\leq 18$ & $\mathrm{~K} 6 \pm 2$ & $1.3 \pm 0.5$ & $0.57 \pm 0.27$ \\
\hline 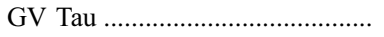 & 1999 Dec 6 & 30 & 5.4 & 7.1 & $0.25 \pm 0.05$ & $13.4 \pm 3.8$ & $25.3 \pm 7.0$ & $\mathrm{~K} 7 \pm 2$ & $1.7 \pm 0.4$ & $1.1 \pm 0.4$ \\
\hline IRAS $04264+2433 \ldots \ldots \ldots \ldots \ldots \ldots \ldots$ & 1999 Dec 5 & 60 & 2.0 & 5.1 & $0.21 \pm 0.14$ & $11 \pm 8$ & $\leq 15:$ & $\mathrm{M} 1 \pm 2$ & 1.4: & $0.99 \pm 0.49$ \\
\hline 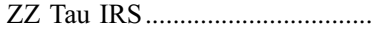 & $2003 \mathrm{Feb} 18$ & 60 & 4.7 & 8.4 & $0.15 \pm 0.06$ & $18.4 \pm 3.7$ & $22 \pm 12$ & $\mathrm{M} 4.5 \pm 2$ & $2.1 \pm 0.7$ & $0.81 \pm 0.29$ \\
\hline \multirow[t]{2}{*}{ L1551 IRS 5} & 1999 Dec 5 & 60 & 0.7 & 0.1 & $\leq 0.4$ & $\ldots$ & $\ldots$ & $\ldots$ & $\ldots$ & $\ldots$ \\
\hline & 2003 Feb 17 & 30 & 0.6 & 1.4 & $\leq 0.5$ & $\ldots$ & $\ldots$ & $\cdots$ & $\ldots$ & $\ldots$ \\
\hline 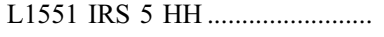 & $2003 \mathrm{Feb} 17$ & 30 & 0.6 & 0.1 & $\leq 0.5$ & $\ldots$ & $\ldots$ & $\ldots$ & $\ldots$ & $\ldots$ \\
\hline L1551 IRS 5 Neb ........................... & 2003 Feb 18 & 60 & 1.0 & 0.3 & $\leq 0.8$ & $\ldots$ & $\ldots$ & $\ldots$ & $\ldots$ & $\ldots$ \\
\hline 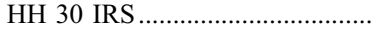 & 2002 Dec 13 & 60 & 9.6 & 8.5 & $0.17 \pm 0.03$ & $20.3 \pm 3.5$ & $\leq 12$ & $\mathrm{M} 0 \pm 2$ & $5.3 \pm 1.0$ & $5.7 \pm 0.9$ \\
\hline 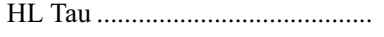 & 1999 Dec 5 & 20 & 29 & 33 & $0.26 \pm 0.02$ & $18.3 \pm 2.6$ & $26.3 \pm 5.2$ & $\mathrm{~K} 5 \pm 1$ & $0.91 \pm 0.07$ & $0.99 \pm 0.15$ \\
\hline IRAS $04295+2251 \ldots \ldots \ldots \ldots$ & 1999 Dec 5 & 60 & 0.1 & 0.8 & $\leq 2.7$ & $\ldots$ & $\ldots$ & $\ldots$ & $\ldots$ & $\ldots$ \\
\hline IRAS $04302+2247 \ldots \ldots \ldots \ldots \ldots \ldots \ldots$ & 1999 Dec 5 & 120 & 0.8 & 1.8 & $\leq 0.4$ & $\ldots$ & $\ldots$ & $\ldots$ & $\ldots$ & $\ldots$ \\
\hline Haro $6-28 \ldots \ldots \ldots \ldots \ldots \ldots \ldots \ldots$ & 2002 Dec 13 & 20 & 28 & 47 & $0.46 \pm 0.02$ & $16.1 \pm 0.3$ & $10.1 \pm 1.0$ & $\mathrm{M} 3.5 \pm 0.5$ & $0.23 \pm 0.05$ & $\leq 0.07$ \\
\hline 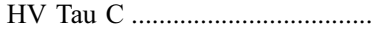 & 2002 Dec 13 & 40 & 21 & 19 & $0.47 \pm 0.02$ & $23.1 \pm 0.6$ & $19.4 \pm 1.9$ & $\mathrm{~K} 6 \pm 1$ & $0.39 \pm 0.07$ & $\leq 0.19$ \\
\hline IC $2087 \ldots \ldots \ldots$ & 2002 Dec 13 & 20 & 2.5 & 14 & $\leq 0.12$ & $22 \pm 8$ & $\leq 15:$ & $\mathrm{K}:$ & 2.7: & $3.2 \pm 0.6$ \\
\hline 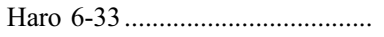 & 2002 Dec 13 & 20 & 14 & 23 & $0.59 \pm 0.02$ & $15.8 \pm 0.6$ & $22.8 \pm 1.6$ & $\mathrm{M} 0.5 \pm 0.5$ & $0.13 \pm 0.06$ & $\leq 0.04$ \\
\hline DP Tau & 2003 Feb 17 & 5 & 47 & 48 & $0.28 \pm 0.02$ & $16.8 \pm 0.6$ & $19.2 \pm 1.3$ & $\mathrm{M} 0 \pm 1$ & $1.8 \pm 0.2$ & $1.3 \pm 0.3$ \\
\hline IRAS $04489+3042 \ldots \ldots \ldots \ldots \ldots \ldots \ldots$ & 1999 Dec 5 & 60 & 0.5 & 2.5 & $\leq 0.6$ & $15 \pm 5$ & $\leq 15:$ & $\mathrm{M} 6 \pm 2$ & 1.3: & $0.56 \pm 0.20$ \\
\hline RW Aur A & 2003 Feb 17 & 1 & 67 & 49 & $0.29 \pm 0.02$ & $16.0 \pm 1.7$ & $34.4 \pm 5.7$ & $\mathrm{~K} 2 \pm 2$ & $1.1 \pm 0.3$ & $1.5 \pm 0.6$ \\
\hline 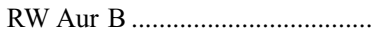 & 2003 Feb 17 & 1 & 14 & 16 & $0.50 \pm 0.02$ & $15.9 \pm 0.5$ & $12.2 \pm 1.6$ & $\mathrm{~K} 6 \pm 1$ & $0.26 \pm 0.12$ & $0.17 \pm 0.13$ \\
\hline
\end{tabular}


TABLE 2-Continued

\begin{tabular}{|c|c|c|c|c|c|c|c|c|c|c|}
\hline Star & Obs. Date & $\begin{array}{c}\text { Exposure } \\
\text { Time } \\
\text { (minutes) }\end{array}$ & $\mathrm{S} / \mathrm{N}_{6700}$ & $\mathrm{~S} / \mathrm{N}_{8450}$ & $\begin{array}{c}\mathrm{EW}[\mathrm{Li} \mathrm{I}] \\
(\AA)\end{array}$ & $\begin{array}{c}\text { Radial } \\
\text { Velocity } \\
\left(\mathrm{km} \mathrm{s}^{-1}\right)\end{array}$ & $\begin{array}{c}v \sin i \\
\left(\mathrm{~km} \mathrm{~s}^{-1}\right)\end{array}$ & $\begin{array}{l}\text { Spectral } \\
\text { Type }\end{array}$ & $r_{6500}$ & $r_{8400}$ \\
\hline \multicolumn{11}{|c|}{ Additional T Tauri Stars in Taurus-Auriga } \\
\hline IRAS $04278+2253 b \ldots \ldots \ldots \ldots \ldots \ldots$ & 2002 Dec 13 & 15 & 14 & 24 & $0.42 \pm 0.02$ & $15.7 \pm 0.4$ & $\leq 7.4$ & $\mathrm{~K} 7 / \mathrm{M} 0 \pm 1$ & $0.61 \pm 0.10$ & $0.49 \pm 0.11$ \\
\hline 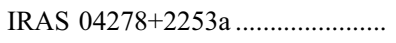 & 2002 Dec 13 & 15 & 15 & 27 & $0.25 \pm 0.02$ & $19.2 \pm 2.7$ & $15 . \overline{7} \pm 4.9$ & $\mathrm{G} 8 \pm 2$ & $0.34 \pm 0.22$ & $0.63 \pm 0.33$ \\
\hline HK Tau A & 2002 Dec 13 & 8 & 23 & 31 & $0.50 \pm 0.02$ & $21.6 \pm 0.5$ & $17.8 \pm 1.1$ & $\mathrm{M} 1 \pm 0.5$ & $0.40 \pm 0.10$ & $0.24 \pm 0.06$ \\
\hline 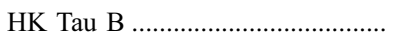 & 2002 Dec 13 & 20 & 12 & 16 & $0.58 \pm 0.02$ & $17.4 \pm 0.3$ & $\leq 7.3$ & $\mathrm{M} 1 \pm 0.5$ & $0.14 \pm 0.06$ & $<0.12$ \\
\hline 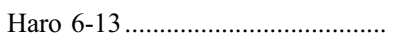 & 2002 Dec 13 & 20 & 24 & 40 & $0.50 \pm 0.02$ & $18.6 \pm 0.8$ & $23.3 \pm 1.1$ & $\mathrm{M} 0 \pm 0.5$ & $0.43 \pm 0.08$ & $0.26 \pm 0.08$ \\
\hline \multirow[t]{2}{*}{ IRAS $04303+2240 \ldots \ldots \ldots \ldots \ldots \ldots \ldots \ldots$} & 1999 Dec 6 & 30 & 53 & 69 & $\leq 0.05$ & & & & 14.9: & 7.9: \\
\hline & 2003 Feb 17 & 20 & 10.3 & 18 & $0.25 \pm 0.03$ & $20.8 \pm 3.6$ & $35 \pm 16$ & $\mathrm{M} 0.5 \pm 1$ & $3.9 \pm 0.4$ & $2.6 \pm 1.1$ \\
\hline DN Tau & 1999 Dec 6 & 20 & 49 & 55 & $0.53 \pm 0.02$ & $16.9 \pm 0.3$ & $9.8 \pm 0.9$ & $\mathrm{M} 0 \pm 0.5$ & $0.24 \pm 0.07$ & $<0.07$ \\
\hline 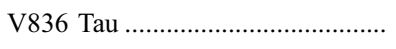 & 1999 Dec 6 & 20 & $71^{\mathrm{a}}$ & 71 & $0.55 \pm 0.02$ & $18.5 \pm 0.3$ & $12.1 \pm 1.0$ & $\mathrm{~K} 7 \pm 1+\mathrm{M}$ & $0.12 \pm 0.10$ & $<0.16$ \\
\hline \multicolumn{11}{|c|}{ T Tauri Stars in the TW Hydrae Association } \\
\hline 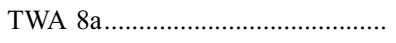 & 2003 Feb 18 & 20 & 195 & 231 & $0.55 \pm 0.02$ & $8.7 \pm 0.2$ & $\leq 5.5$ & $\mathrm{M} 3 \pm 0.5$ & $\leq 0.08$ & $\leq 0.08$ \\
\hline 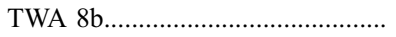 & 2003 Feb 18 & 20 & 44 & 88 & $0.63 \pm 0.02$ & $8.2 \pm 0.2$ & $10.8 \pm 1.0$ & M5.5 \pm 0.5 & $\leq 0.06$ & $\leq 0.17$ \\
\hline TWA 9a & 2003 Feb 18 & 18 & 239 & 190 & $0.53 \pm 0.02$ & $10.9 \pm 0.3$ & $11.1 \pm 1.0$ & $\mathrm{~K} 7 \pm 1$ & $\leq 0.09$ & $\leq 0.10$ \\
\hline 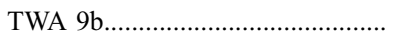 & 2003 Feb 18 & 18 & 63 & 88 & $0.54 \pm 0.02$ & $12.2 \pm 0.4$ & $9.0 \pm 1.0$ & $\mathrm{M} 3.5 \pm 0.5$ & $\leq 0.20$ & $\leq 0.15$ \\
\hline
\end{tabular}

${ }^{a}$ The increasing strength of TiO bands toward the reddest wavelengths suggests that the spatially unresolved companion stars in these systems are spectral class M. 

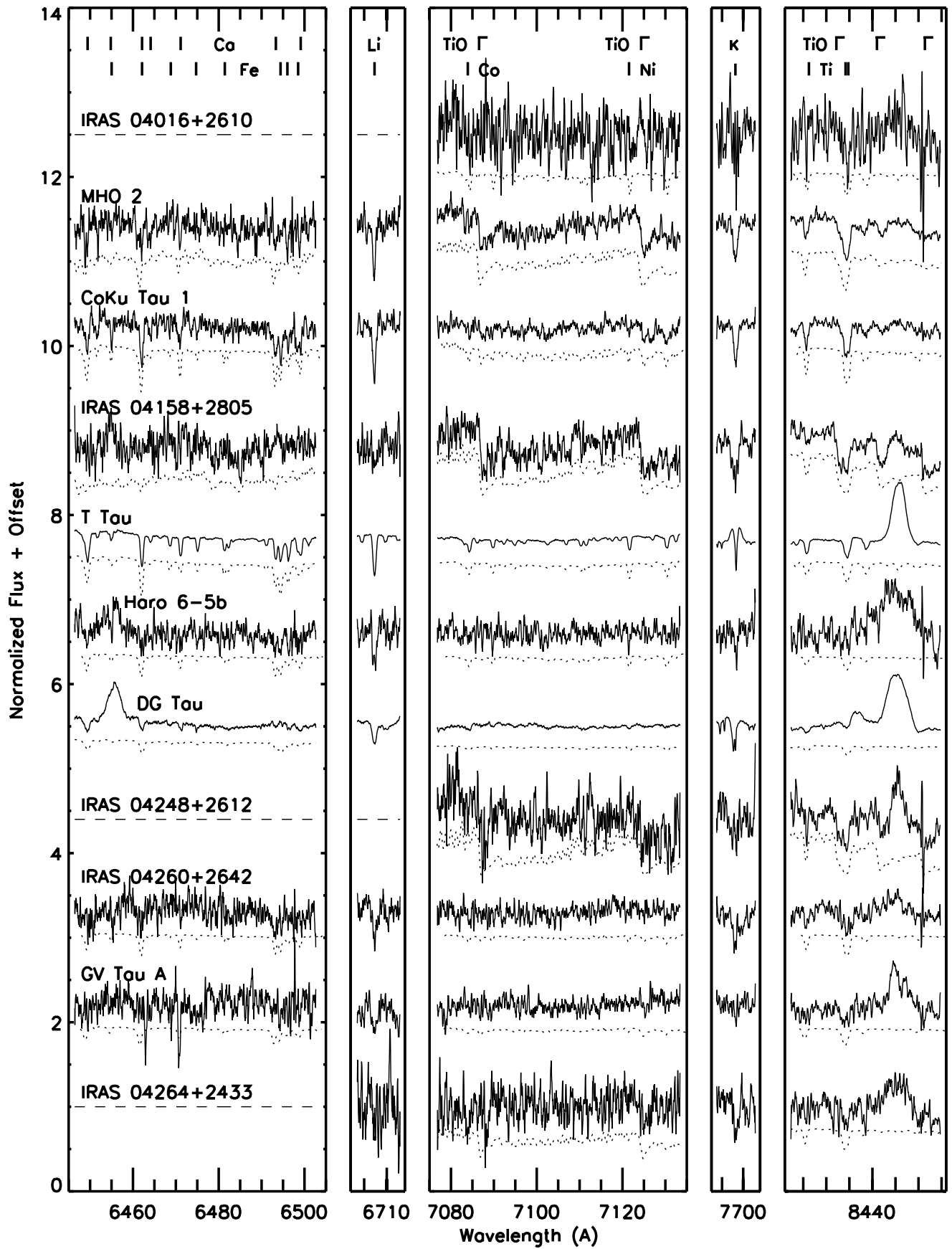

FIG. 2.- Spectral segments obtained with Keck/HIRES of environmentally young stars. Only stars with S/N $>2$ are plotted; portions of spectra with S/N $<2$ are shown as dashed lines. The 6445-6500, 7060-7130, 8420-8455 ̊̊ regions are the primary ones used to determine the spectral types and veiling; the best fit dwarf spectral standards, rotationally broadened and veiled, are shown for each star. The youth diagnostic Li I $\lambda 6708$ and the gravity-sensitive feature $\mathrm{K}$ I $\lambda 7699$ are also shown; these are typically the strongest and most easily identifiable lines in low-S/N optically veiled spectra. Several optically veiled, accreting stars show Fe II $\lambda 6456$ and $\mathrm{O}$ I $\lambda 8446$ emission.

spectra. No weak-lined T Tauri stars (class III stars) are included.

\subsection{Measured Spectral Properties \\ 3.1.1. Radial and Rotational Velocities}

Radial and projected rotational velocities are determined via a cross-correlation analysis (e.g., Hartmann et al. 1986). First, we identify up to 10 spectral regions of length $20-30 \AA$ with sufficient $\mathrm{S} / \mathrm{N}(>2)$ and without prominent telluric absorption features (e.g., Tinney \& Reid 1998), strong gravity-sensitive features (K I $\lambda \lambda 7665,7699, \mathrm{Na}$ I $\lambda \lambda 8183,8195, \mathrm{Ca}$ II $\lambda 8498)$, or emission lines. These regions are cross-correlated with at least three spectral standards of spectral type similar to the target star (see § 3.1.2) and with radial velocities accurate to 0.3$0.4 \mathrm{~km} \mathrm{~s}^{-1}$ (Nidever et al. 2002).

To determine the final radial velocities of the stars, the velocity offsets from the standards must be corrected for barycentric velocity corrections and possible errors in the wavelength solution of either the standard or target spectrum. The former is determined using the rvcorrect task in IRAF. The latter correction may only be relevant when combining data from multiple 

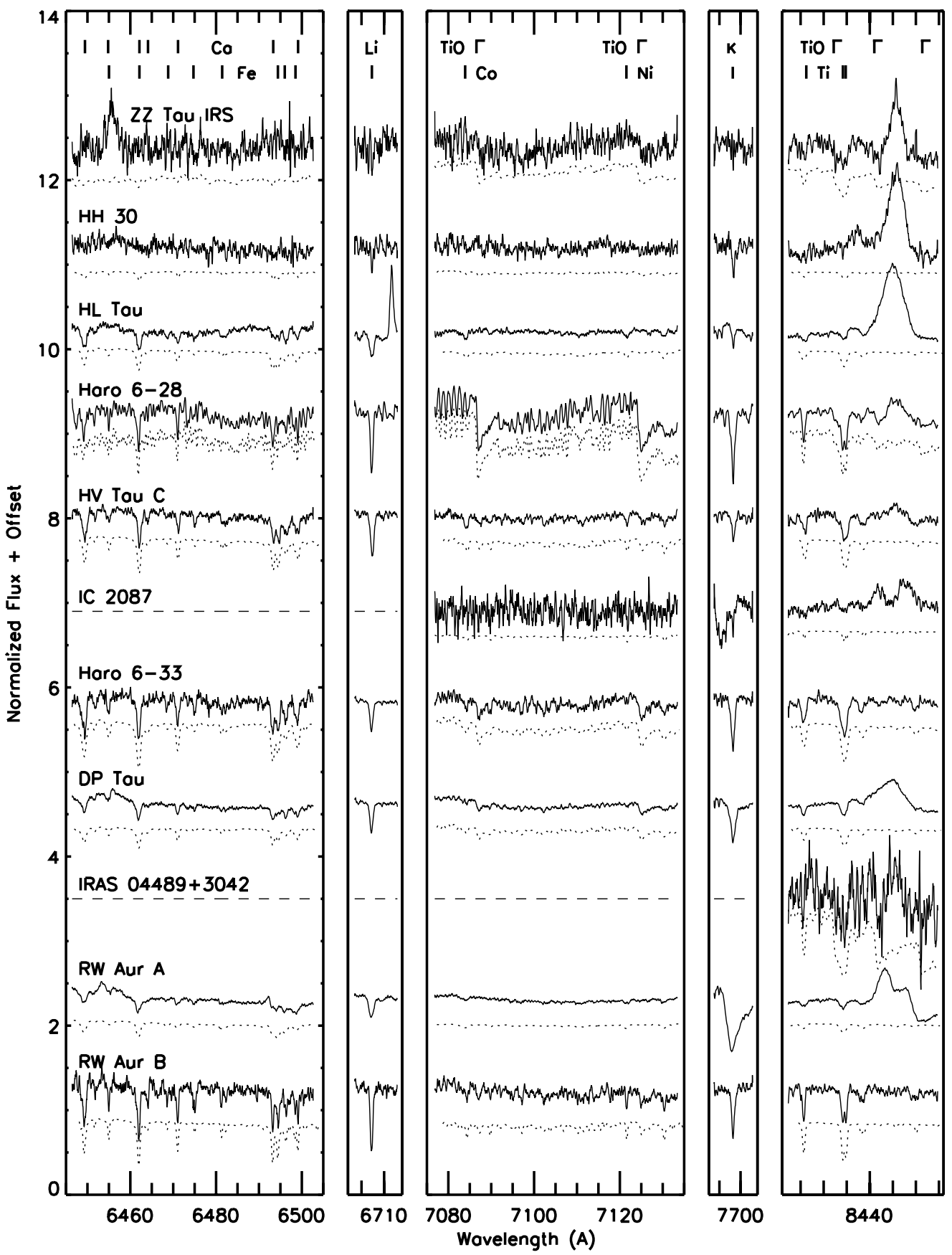

FIG. 2.-Continued

nights and/or multiple runs, as is the case here. This correction is determined by cross-correlating the telluric A band of the standard and the target star. Failure to correct for this effect leads to radial velocity errors of $3-4 \mathrm{~km} \mathrm{~s}^{-1}$. Correcting for this effect yields radial velocities of the standards that are self-consistent to better than $0.5 \mathrm{~km} \mathrm{~s}^{-1}$, consistent with the accuracy of their radial velocities. Uncertainties are estimated by adding, in quadrature, the standard deviation of the multiple radial velocity estimates (3-7 standards are used in each case) and the uncertainty in each radial velocity estimate, which is assumed to be the error in the mean of all spectral regions used per star.

Rotational velocities $(v \sin i)$ for the observed targets are determined from the width of the peak in their cross-correlation with a slowly rotating standard. The width is estimated by fitting a Gaussian plus quadratic function to the cross-correlation peak. The width versus $v \sin i$ relation is determined empirically by cross-correlating a "spun up" spectrum of a slowly rotating standard. The rotationally broadened spectra are constructed using the profiles given in Gray $(1992 ; \epsilon=0.6)$. As has been noted in previous work (e.g., White \& Basri 2003), the presence of a continuum excess (see $\S 3.1 .3$ ) has little effect on the inferred projected rotational velocities, as long as the features are sufficiently well measured. Uncertainties in $v \sin i$ are determined following the same procedure used for the radial velocities. We find that all templates within a few spectral subclasses of a star generally give consistent $v \sin i$ values; an exact match in spectral type is not critical in determining $v \sin i$. However, differences in spectral type and luminosity class can affect $v \sin i$ 

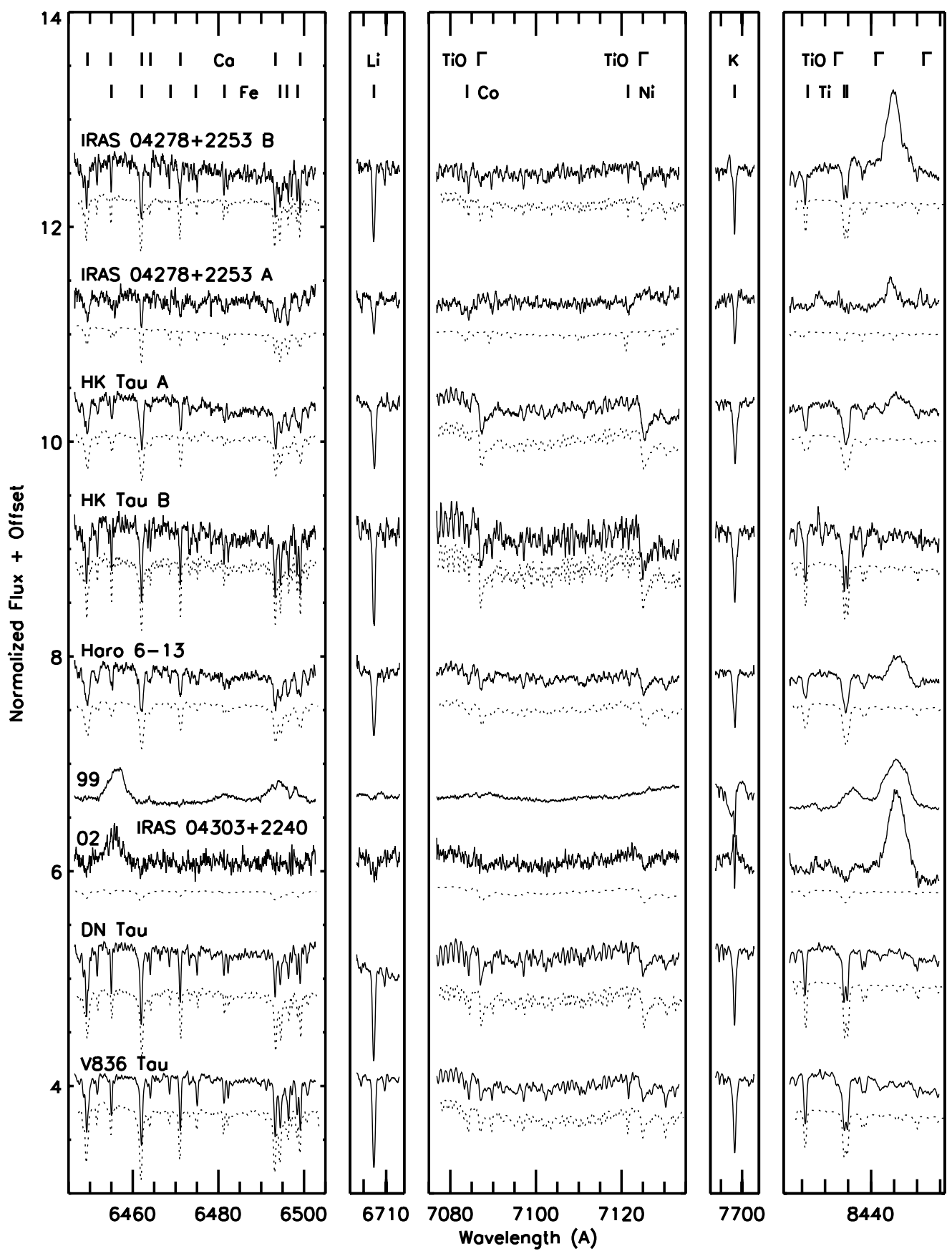

Fig. 3.-Spectral segments obtained with Keck/HIRES of additional T Tauri stars not meeting the environmentally young criteria. Features are as in Fig. 2. Spectra from 2 epochs are shown for IRAS $04303+2240$ (see Table 2). This star was both brighter and more optically veiled during the first epoch.

measurements at low rotational velocities (less than $\sim 10 \mathrm{~km} \mathrm{~s}^{-1}$; see White \& Basri 2003). To avoid introducing this bias, we adopt a conservative approach in determining $v \sin i$ upper limits. If the uncertainty is less than 1 standard deviation from the theoretical $v \sin i$ detection limit $\left(7-15 \mathrm{~km} \mathrm{~s}^{-1}\right.$, set by $\left.\mathrm{S} / \mathrm{N}\right)$, the measurement is assigned an upper limit.

The described procedure worked well for determining radial and projected rotational velocities for 24 of the 28 stars with a continuum detection. Because of low $\mathrm{S} / \mathrm{N}$ and, in some cases, high veiling ( $\S 3.1 .2$ ), the cross-correlation analysis for the remaining four stars (IRAS 04016+2610, IRAS 04264+2433, IC 2087, and IRAS 04489+3042) was successful in only a few segments (2-3) of their spectra. The correlation results for these four stars imply no measurable rotation but do yield radial velocities that are consistent with that of Taurus. In the cases of IRAS $04016+2610$ and IC 2087 , we are especially cautious about the results. The photospheric-like features in their spectra are quite weak and/or noisy and possibly could be spurious noise events. The results for IRAS $04264+2433$ and IRAS $04489+3042$ are slightly more robust, however, since both stars show TiO absorption bands that can be more confidently identified (see Figs. 1 and 2). The radial velocities and $v \sin i$ detection limits $\left(15 \mathrm{~km} \mathrm{~s}^{-1}\right)$ for these stars are listed in Table 2 followed by colons to indicate possible larger systematic uncertainties.

Although for the majority of these stars their measurements are the first epoch of high-resolution spectroscopy, no definitive spectroscopic binaries are identified. With the exception of 

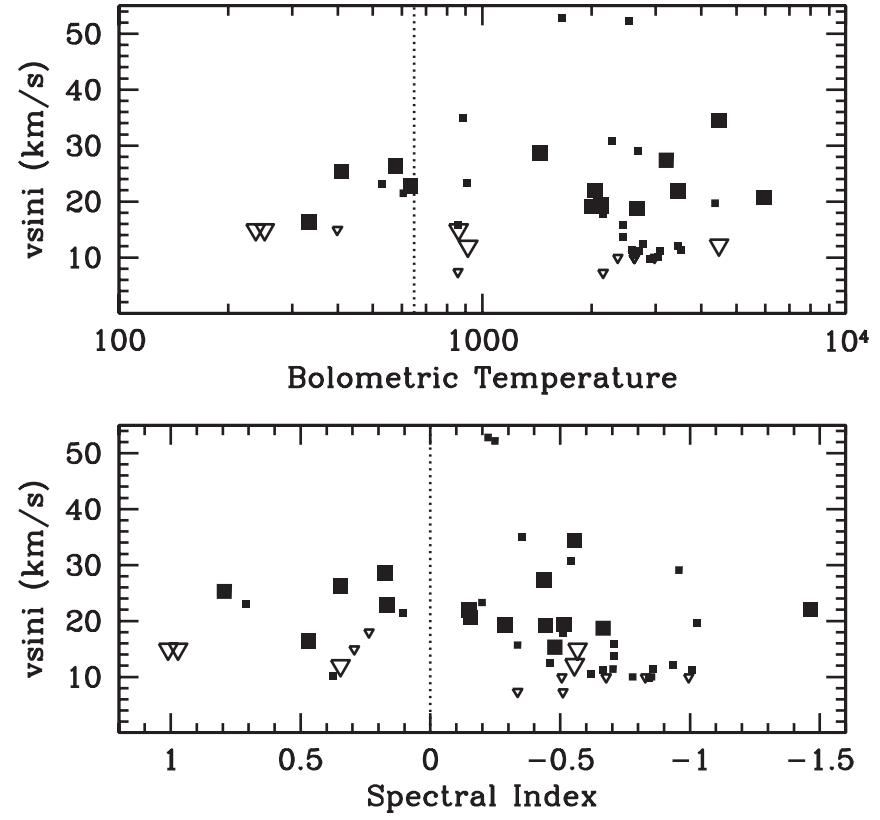

FIG. 4.-Projected rotational velocities $(v \sin i)$ vs. bolometric temperature (top panel) and spectral index (bottom panel). The vertical dotted lines are proposed values for separating class I and class II stars (see $\S 2.1$ ). Large symbols indicate stars that power HH flows (i.e., HH stars). Open symbols are $v \sin i$ upper limits. The stellar rotational velocities do not evolve significantly with either evolutionary diagnostic. HH stars rotate slightly faster than non$\mathrm{HH}$ stars, on average.

HV Tau C, all stars have radial velocities within $2 \sigma$ of the mean of Taurus-Auriga (17.4 \pm 2.1 ; Hartmann et al. 1986) and thus are consistent with being Taurus members. The radial velocity of HV Tau C $(23.1 \pm 0.6)$ is $2.6 \sigma$ from the mean of Taurus, and thus the star could be a single-lined spectroscopic binary. Unfortunately, we do not have a spectrum of its companions HV $\mathrm{Tau} \mathrm{AB}$ for a more strict comparison. We also note that radial velocities of HK Tau A and HK Tau B are significantly different $(21.6 \pm 0.5$ and $17.4 \pm 0.3)$, several times that expected from relative orbital motion alone. We suggest either that one of these stars is a single-lined spectroscopic binary or that the pair is not physically associated. The latter case would explain their apparently non-coplanar disks (Ducheñe et al. 2003).

The projected rotational velocities of the environmentally young star sample are relatively slow, ranging from 10 to $34 \mathrm{~km} \mathrm{~s}^{-1}$. The measured $v \sin i$ values of this sample and the additional sample of class II stars are plotted in Figure 4 versus the evolutionary diagnostics $T_{\text {bol }}$ and $\alpha$. The environmentally young stars have measured $v \sin i$ values (excluding upper limits) that are generally quite similar to the values of environmentally older stars. Treating $v \sin i$ upper limits as detections, the distributions of $v \sin i$ values for class I and class II stars are similar (K-S probability of 0.079), although the median $v \sin i$ of class I stars is slightly larger than that of class II stars (median class I $v \sin i=18.0 \mathrm{~km} \mathrm{~s}^{-1}, \sigma=5.1$; median class II $v \sin i=14.4 \mathrm{~km} \mathrm{~s}^{-1}, \sigma=10.8$ ). Comparing $\mathrm{HH}$ stars and non-HH stars, the distributions of $v \sin i$ are more discrepant (K-S probability of 0.0034 ; a $2.9 \sigma$ difference); HH stars rotate slightly faster than non-HH stars (median $v \sin i=19.4 \mathrm{~km} \mathrm{~s}^{-1}, \sigma=5.9$; median $v \sin i=12.1 \mathrm{~km}$ $\mathrm{s}^{-1}, \sigma=11.5$ ). Table 3 summarizes the median statistics, excluding brown dwarfs.

\subsubsection{Spectral Types and Continuum Excesses}

Spectral types and continuum excesses are determined by matching the line/band ratios and line/band depths, respectively, to those of rotationally broadened and artificially veiled dwarf spectral standards. The procedure works as follows for a given star. First, all of the comparison dwarf spectral standards are rotationally broadened to have the same $v \sin i$ as the young star. For each rotationally broadened standard, a set of "veiled" templates are then generated by adding a range of continuum excess values, all assumed to be constant over each $\sim 20 \AA$ spectral region. This set of rotationally broadened and veiled templates are then compared with the young star sample to determine which spectral type gives the best match. Quantitatively, the best match is determined by minimizing the rms values over three temperature-sensitive regions: 6490-6502, 7120-7128, and 8431-8436 $\AA$ (see Figs. 2 and 3). In most cases, the assigned spectral type is that of the spectral standard that gives the best fit. In some cases, however, emission lines or anomalous low-S/N features yield clearly discrepant spectral types. Consequently, all spectral types are confirmed by visual inspection. Uncertainties in the spectral type are determined by the range of spectral types that can reasonably match the spectrum; the uncertainties range from 0.5 to 3 spectral subclasses. More robust techniques for determining temperatures such as synthetic modeling of the spectra are currently unwarranted given the low $\mathrm{S} / \mathrm{N}$ of the data. The low S/N of IRAS $04016+2610$ and the high optical veiling of IC 2087 prevent us from determining their spectral types. However, both show $\mathrm{Ti}$ I $(\lambda \lambda 8435,8436)$ absorption that is stronger than $\mathrm{Nb}$ I (28439) absorption, suggesting a spectral type cooler than $\mathrm{G}$. The absence of any clear TiO bands suggests a spectral type hotter than M. We classify these two stars as K stars. The inferred spectral types for all stars are listed in Table 1.

The inferred spectral types range from K0 to M6 for the environmentally young star sample. Class I stars and class II stars have a similar distribution of stellar spectral types. HH stars, on the other hand, tend to be slightly hotter than non-HH stars. Of the 46 stars studied here with spectral types hotter than M3.5, 20 are HH stars (43\%). In contrast, of the nine stars with spectral types of M3.5 or cooler, only two are HH stars (22\%).

Once the spectral type of the star is determined, the continuum excess, called $r$ and defined as $r=F_{\text {ex }} / F_{\text {phot }}$, is determined by

TABLE 3

Summary of Median Properties, Substellar Objects Excluded

\begin{tabular}{|c|c|c|c|c|c|c|c|c|c|c|c|c|c|c|}
\hline Type & $\begin{array}{c}v \sin i \\
\left(\mathrm{~km} \mathrm{~s}^{-1}\right)\end{array}$ & $N^{\mathrm{a}}$ & $r_{6500}$ & $N^{\mathrm{a}}$ & $\begin{array}{c}\mathrm{EW}[\mathrm{H} \alpha] \\
(\AA)\end{array}$ & $N^{\mathrm{a}}$ & $\begin{array}{c}\mathrm{H} \alpha 10 \% \text { Width } \\
\left(\mathrm{km} \mathrm{s}^{-1}\right)\end{array}$ & $N^{\mathrm{a}}$ & $\begin{array}{c}\text { EW[S II] } \\
(\AA)\end{array}$ & $N^{\mathrm{a}}$ & $\begin{array}{c}\log \dot{M}_{\mathrm{acc}} \\
\left(M_{\odot} \mathrm{yr}^{-1}\right)\end{array}$ & $N^{\mathrm{a}}$ & $\begin{array}{c}\log \dot{M}_{\text {out }} \\
\left(M_{\odot} \mathrm{yr}^{-1}\right)\end{array}$ & $N^{\mathrm{a}}$ \\
\hline Class Is......... & $19.8 \pm 5.7$ & 8 & $0.6 \pm 0.6$ & 8 & $-51 \pm 33$ & 13 & $344 \pm 131$ & 13 & $-2.4 \pm 5.1$ & 13 & $-7.1 \pm 0.8$ & 8 & $-7.0 \pm 1.5$ & 8 \\
\hline $\mathrm{HH}$ & $19.4 \pm 6.0$ & 19 & $1.0 \pm 1.4$ & 23 & $-61 \pm 69$ & 24 & $409 \pm 120$ & 21 & $-1.8 \pm 22$ & 21 & $-7.0 \pm 0.6$ & 23 & $-7.2 \pm 0.9$ & 20 \\
\hline non-HH ........ & $11.4 \pm 11.9$ & 31 & $0.5 \pm 1.1$ & 34 & $-58 \pm 37$ & 37 & $414 \pm 116$ & 38 & $-0.1 \pm 3.4$ & 33 & $-7.4 \pm 0.7$ & 35 & $-8.7 \pm 1.0$ & 30 \\
\hline
\end{tabular}

${ }^{a} \mathrm{~N}$ indicates the number of stars used to determine the median and standard deviation. 


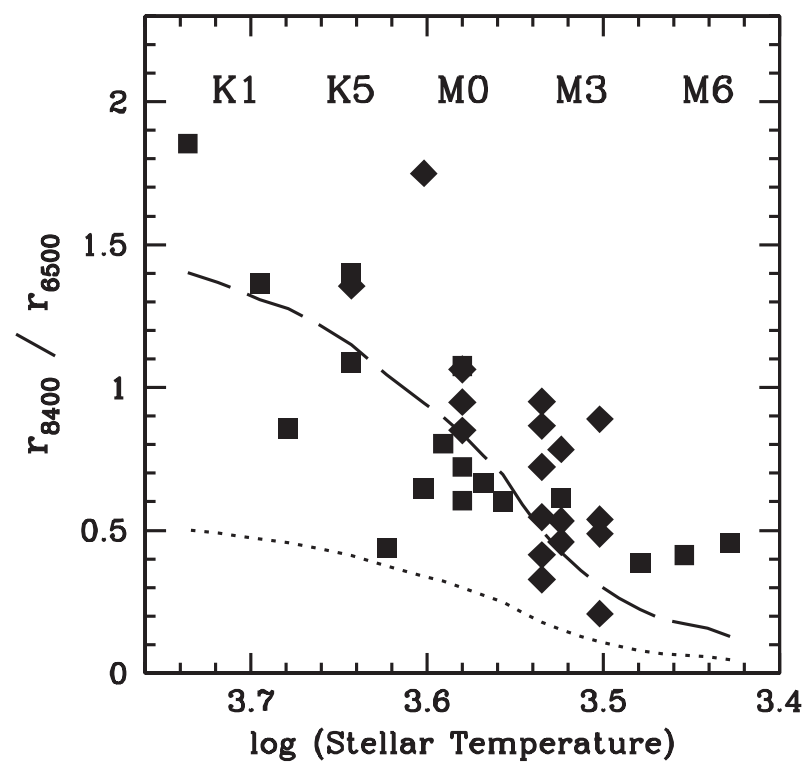

FIG. 5.-Ratio of optical veiling at $8400 \AA$ to that at $6500 \AA$ vs. stellar temperature. Squares are ratios determined from values listed in Table 2 and from White \& Basri (2003); diamonds are determined from the values in $\left(r_{6110}\right.$ to $r_{8115}$ for these points) Hartigan \& Kenyon (2003). The observed veiling ratios are more consistent with a continuum excess of constant flux $\left(F_{\mathrm{ex}}=C\right.$; dashed line) over this wavelength interval, than with a Rayleigh-Jeans approximation $\left(F_{\mathrm{ex}} \propto \lambda^{-4} ;\right.$ dotted line $)$, as would be expected if the excess originated from a spot much hotter than the stellar photosphere. We interpret this as evidence for a second, cooler component to the accretion-generated excess emission.

comparing the depths of photospheric features with those of a rotationally broadened and artificially veiled dwarf spectrum of the same spectral type. The continuum excess added to the standard is allowed to be negative; this results in a comparison standard with deeper absorption features. Consequently, stars with no continuum excess should have values of $r$ that scatter about zero, and this scatter can be used to estimate the uncertainty in $r$. The continuum excess is determined in two spectral regions, 6500 and $8400 \AA$, by averaging the results within $50 \AA$ of these wavelengths. The uncertainties in the continuum excess are estimated by changing the rotationally broadened and veiled template by the spectral type uncertainty and redetermining the continuum excess; the spectral type uncertainty typically dominates the uncertainty in the continuum excess. If the uncertainty in the continuum excess is larger than the measured value, the uncertainty is assigned as an upper limit to detection. As a check of our procedure, we note that the four weak-lined T Tauri stars TWA 8a, TWA 8b, TWA 9a, and TWA 9b, with spectral types ranging from $\mathrm{K} 7$ to $\mathrm{M} 5.5$, can be successfully modeled using a rotationally broadened dwarf spectrum without any continuum excess. For the K stars IRAS 04016+2610 and IC 2087, we use a $\mathrm{K} 4$ dwarf as the template from which the veiling is determined. The inferred continuum excess values at $6500 \AA\left(r_{6500}\right)$ and at $8400 \AA\left(r_{8400}\right)$ are listed in Table 2 . For five stars, the $\mathrm{S} / \mathrm{N}$ is insufficient to determine the continuum excess at $6500 \AA$ but is sufficient to measure the excess at $8400 \AA$.

Figure 5 shows the ratio of the continuum veiling at $8400 \AA$ to the continuum veiling at $6500 \AA$ as a function of stellar effective temperature. Values plotted include measurements from this study ( Table 2) and from White \& Basri (2003). In addition, we show the ratio of optical veiling values from Hartigan \& Kenyon (2003), determined from similar but slightly shorter wavelength intervals $\left(r_{6100} / r_{8115}\right)$, using low-resolution spectra; only veiling values above 0.1 are included from this data set to avoid spuriously large ratios. For early- to mid-K stars, the continuum excesses at $8400 \AA$ are in many cases larger than the values determined at $6400 \AA$. This is somewhat surprising, since the optical excesses are generally attributed to featureless continuum emitted by a $\sim 10^{4} \mathrm{~K}$ hot spot(s) (e.g., Kenyon et al. 1994; Calvet $\&$ Gullbring 1998) and therefore should be significantly less at $8400 \AA$. We note that this effect is consistent with previous veiling studies, which find the veiling to be "almost flat" in the red (Basri \& Batalha 1990); this previous work primarily focused on K5-M0 type stars for which an excess of constant flux would yield nearly a constant veiling value $\left(r_{8400} / r_{6500} \sim 1.0\right.$; Fig. 5). We postpone discussion of this interesting phenomenon until $\S 3.3 .1$ but use these empirical ratios to estimate the continuum excess at $6500 \AA$ for the five stars with a $r_{8400}$ measurement but no $r_{6500}$ measurement. For the two K stars, $r_{6500}$ is estimated to be $r_{8400} / 1.2$; for the M1 star, $r_{6500}$ is estimated to be to be $r_{8400} / 0.70$; for the M5.5 and M6 stars, $r_{6500}$ is estimated to be $r_{8400} / 0.43$. These estimated $r_{6500}$ values are listed in Table 2 followed by colons.

For IRAS 04303+2240, which was observed on both 1999 December 6 and 2003 February 17, no photospheric features were detected during the first epoch, but early $\mathrm{M}$ spectral features were clearly identified during the second (Fig. 1). The star was also much brighter during the first epoch than the second epoch; the relative counts in the spectra suggest a brightness decrease by factors of 3.25 at $6500 \AA$ and 2.47 at $8400 \AA$ (see $\mathrm{S} / \mathrm{N}$ in Table 2). Although no continuum excesses could be measured in the first epoch, since the photosphere is not detected, we estimate the excesses during this epoch by assuming that the change in brightness between the two observations is caused only by a change in the continuum excess. These larger first epoch values are listed in Table 2 and followed by colons.

The distribution of continuum excesses at $6500 \AA\left(r_{6500}\right)$ is shown in Figure 6 as a function of $T_{\text {bol }}$ and $\alpha$. Values for the additional sample of class II stars are also shown, with
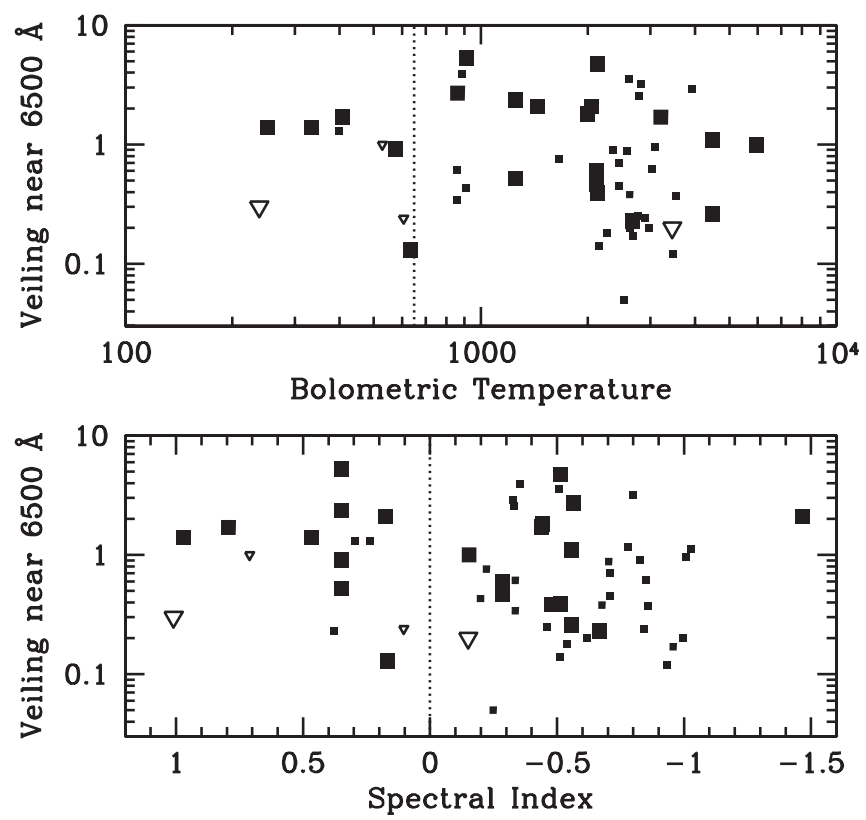

FIG. 6.-Optical veiling near $6500 \AA$ ( $r_{6500}$ in Table 2$)$ vs. bolometric temperature (top panel) and spectral index (bottom panel). The symbols are the same as in Fig. 4. The amount of excess emission does not evolve significantly with either evolutionary diagnostic. HH stars and non-HH stars have similar excesses. 
continuum excess measurements from Basri \& Batalha (1990), Hartigan et al. (1995), and White \& Basri (2003). Environmentally young stars have optical veiling values that are similar to those of environmentally older stars. K-S tests show no differences in the distributions, but slight differences are seen in the median values. The continuum excesses of the 11 class I stars (median $r_{6500}=1.0, \sigma=0.6$ ) are marginally larger than the continuum excesses of the 50 class II stars (median $r_{6500}=$ $0.6, \sigma=1.3$ ). Similarly, the continuum excesses of the $24 \mathrm{HH}$ stars (median $r_{6500}=1.1, \sigma=1.3$ ) are marginally larger than the continuum excesses of the 36 non-HH stars (median $r_{6500}=$ $0.6, \sigma=1.0$ ). Table 3 summarizes the median statistics, excluding brown dwarfs.

\subsubsection{Surface Gravity Indicators}

Pressure-sensitive photospheric features offer a means of determining whether the surface gravities of class I stars are less than those of class II stars for a given mass. This would indicate that they have larger radii and hence younger ages. Some of the more distinguishing pressure-sensitive features for $\mathrm{K}$ and $\mathrm{M}$ spectral types include $\mathrm{CaH}(\lambda \lambda 6382,6389,6903-6946), \mathrm{K}$ I ( $\lambda \lambda 7665,7699), \mathrm{Na}$ I $(\lambda \lambda 8183,8195), \mathrm{Ca}$ II $(\lambda \lambda 8498,8542$, 8662), and $\mathrm{Fe} \mathrm{I}$ lines (see, e.g., Kirkpatrick et al. 1991). The molecular absorption bands of TiO and $\mathrm{VO}$ present in $\mathrm{M}$ dwarfs are also known to be pressure sensitive (Torres-Dodgen \& Weaver 1993; White \& Basri 2003). Our spectra include K I (27699), Ca II ( $\lambda 28498,8662)$, several $\mathrm{Fe} \mathrm{I}$ lines, and $\mathrm{CaH}$ and $\mathrm{TiO}$ absorption bands. Of these, $\mathrm{Ca}$ II is not a useful diagnostic of surface gravity, since it often shows either strong emission from accretion activity or core emission from chromospheric activity.

The spectra shown in Figures 2 and 3 include the K I at $7699 \AA$. This feature is one of the strongest and most easily identifiable absorption features in our modest $\mathrm{S} / \mathrm{N}$ optically veiled spectra. However, many stars show nonphotospheric components to this feature, including both broad emission profiles with narrow absorption superposed (e.g., DG Tau, HL Tau, and T Tau) and broad asymmetric absorption with possible emission (e.g., IRAS $04303+2240$, IC 2087, and RW Aur A). For objects with no obvious nonphotospheric emission, however, the wing strengths of $\mathrm{K}$ I for both class I and class II stars are intermediate between those of narrow giants and those of pressure-broadened dwarfs. This is a well-known characteristic of T Tauri stars.

For early- and mid-M spectral types, we find that dwarfs, as opposed to giants or some combination of the two, provide a better match to the pressure-sensitive $\mathrm{Fe}$ I lines and $\mathrm{TiO}$ bands of both class I and class II stars (see also White \& Basri 2003). In addition, $\mathrm{CaH}$ bands at 6382 and $6389 \AA$ are clearly seen in many of the class I and class II stars observed here. These features are strongest in the spectra of early $M$ dwarfs and absent in spectra of similar spectral type giants.

The combined results of these comparisons suggest that the surface gravities of both class I and class II stars are intermediate between those of dwarfs and giants but somewhat more similar to dwarfs. This is consistent with the predicted surface gravities for young stars (e.g., Siess et al. 2000), whose radii should be only a few times their main-sequence values, as opposed to $\sim 100$ times their main-sequence values in the case of giant stars. Theoretical predictions suggest class I stars should have radii less than 2 times those of T Tauri stars (e.g., Stahler 1988), which translates into differences in surface gravity of less than a few tenths of a dex. Given the relatively low $\mathrm{S} / \mathrm{N}$ of the class I spectra, measurements of these small $\log g$ differences are not possible from line profile analysis. We are also cautious of such an approach, given the nonphotospheric emission associated with gravity-sensitive features ( $\mathrm{K}_{\text {I }} 27699$; $\$ 3.1 .4$ ). Analyses that focus on determining surface gravities from molecular band strengths (e.g., Mohanty et al. 2004) offer a more promising approach. With the current data at hand, we can neither prove nor disprove that class I stars have lower surface gravities, as would be expected if they are younger than class II stars.

\subsubsection{Prominent Emission Lines}

The emission lines of young stars are powerful probes of the mass accretion and mass outflow processes. Their ratios characterize temperatures and densities of the emitting regions, and their intensities and velocity profiles trace the flow of circumstellar material (e.g., Edwards et al. 1987; Hamann \& Persson 1992; Hamann 1994; Hartigan et al. 1995). Figure 7 shows emission-line profiles of the observed Taurus stars in Table 2. The plotted profiles include the two permitted lines $\mathrm{H} \alpha \lambda 6563$ and $\mathrm{Ca}$ II $\lambda 8498$ and the three forbidden emission lines [O I] $\lambda 6364,\left[\mathrm{~N}\right.$ II] $\lambda 6583$, and [S II] $\lambda 6731$. Fe II $\lambda 6456, \mathrm{~K}_{\mathrm{I}} \lambda 7699$, and $\mathrm{O}_{\text {I }} 28446$ emission can be seen in the spectra plotted in Figures 2 and 3, when present.

Equivalent widths (EWs) of $\mathrm{H} \alpha, \mathrm{Ca}$ II $28498, \mathrm{Ca}$ II 28662 , [O I] $\lambda 6364,\left[\mathrm{~N}_{\text {III }}\right] \lambda 6583$, [S II] $\lambda 6716$, and [S II] $\lambda 6731$ are listed in Table 3. These EWs include emission at all velocities, which, for several forbidden lines, means summing distinct velocity components. In cases where the $\mathrm{Ca}$ II emission appears in the center of the photospheric absorption feature ( $\mathrm{CoKu}$ Tau 1, Haro 6-28, Haro 6-33, RW Aur B, HK Tau A and B, DN Tau, and V836 Tau), the EWs are estimated from the approximate bottom of the stellar absorption profile; no corrections for the stellar absorption are made for stronger emission-line stars. Detection limits are estimated on the basis of the $\mathrm{S} / \mathrm{N}$ of the spectra and/or the quality of the sky subtraction for telluric sensitive features (e.g., [O I] 26364$)$. In cases in which an emission line is present but the $\mathrm{S} / \mathrm{N}$ in the continuum is less than 1.0, the equivalent width is assumed to be a lower limit (up to $999 \AA$ ). Lower limit EW measurements of [S II] $\lambda 6716$ and $\lambda 6731$ use the same continuum value to preserve the ratio of these neighboring features. For stars with $\mathrm{S} / \mathrm{N}<1.0$ and no emission lines, no limits are given. Uncertainties in the EWs are generally $10 \%$ of the listed value and usually limited by the difficulty in determining the continuum level of a cool type photosphere underneath an emission line with broad wings. The EWs of [O I] $\lambda 6364$ and to a lesser extent [S II] $\lambda 6716$ have larger uncertainties $(10 \%-$ $25 \%$ ) because of the uncertain removal of sky emission and stellar absorption features at or near those wavelengths. In several cases, poor sky subtraction yielded lower limits on the EW measurements.

\subsubsection{1. $\mathrm{H} \alpha$ Emission}

Broad $\mathrm{H} \alpha$ emission is one of the most ubiquitous characteristics of mass accretion. Measurements of the line profiles potentially offer a means to characterize the accretion velocity and mass accretion rate (e.g., Muzerolle et al. 1998). $\mathrm{H} \alpha$ emission is detected from all stars observed, albeit marginally for IRAS $04154+2823$ and IRAS $04295+2251$. Figure 8 shows the distributions of $\mathrm{H} \alpha$ EWs versus $T_{\text {bol }}$ and $\alpha$ for the environmentally young star sample and the additional class II star sample. For illustrative clarity, no lower limits are plotted (typical of stars with no continuum detection); since all stars show $\mathrm{H} \alpha$ emission, there are no upper limits. Environmentally young and old stars have indistinguishable $\mathrm{H} \alpha \mathrm{EW}$ distributions, according to K-S tests. The median $\mathrm{EW}[\mathrm{H} \alpha] \mathrm{s}$ of the 16 class I stars and the 49 class II stars are both $-59 \AA$. The median EW[H $\alpha]$ of the $25 \mathrm{HH}$ stars is $-63 \AA$ and is similar to that of the 39 non- $\mathrm{HH}$ 

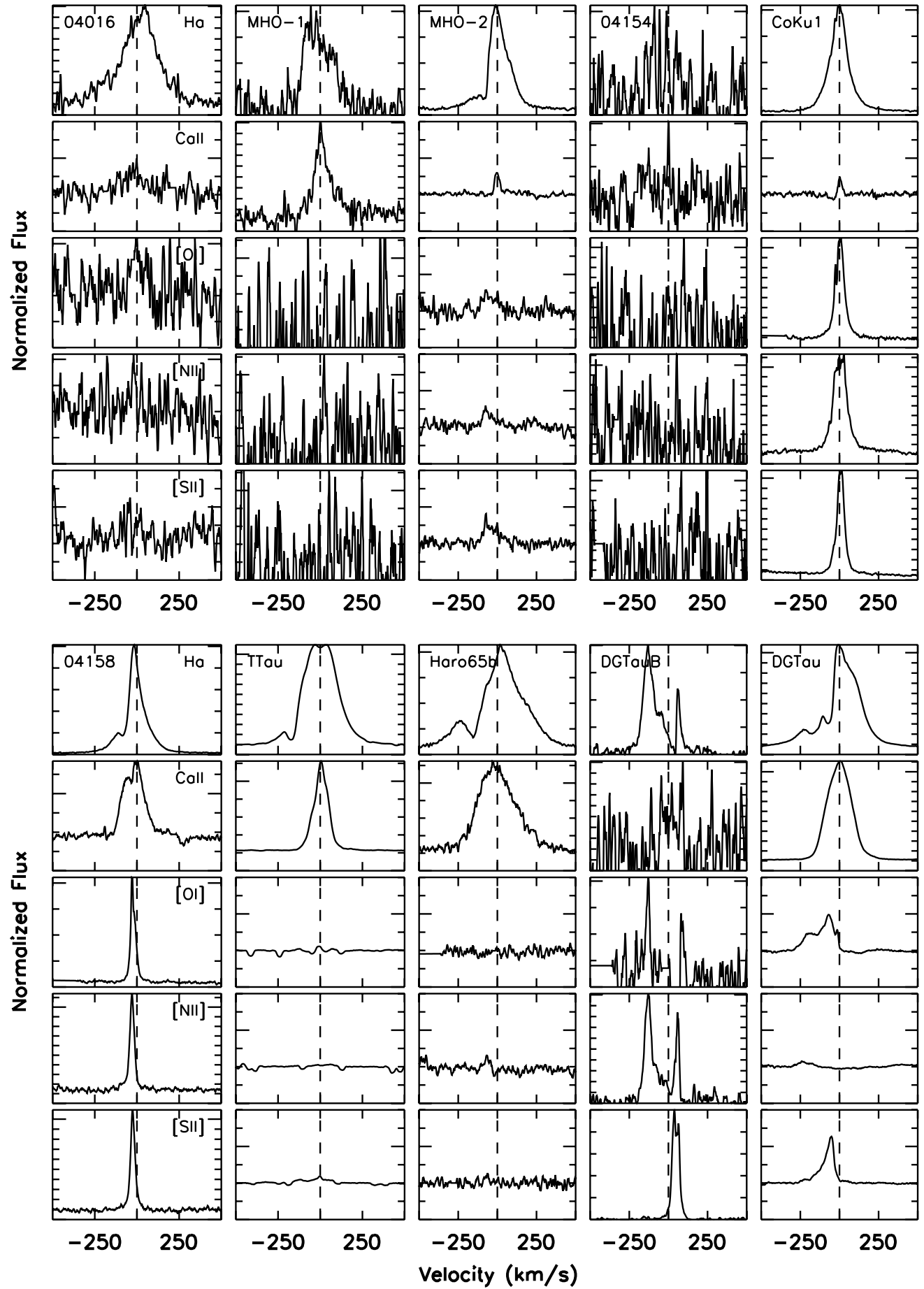

FIG. 7. - $\mathrm{H} \alpha, \mathrm{Ca}$ II $\lambda 8498$, [O I] $\lambda 6364$, [N II] $\lambda 6583$, and [S II] $\lambda 6731$ emission lines of the observed sample (Table 2 ). All spectra are shifted in wavelength to the mean systemic velocity of Taurus $\left(18 \mathrm{~km} \mathrm{~s}^{-1}\right.$; vertical dashed line $)$ and normalized to unity in the continuum. The vertical scale runs from 0.0 to 3.0 , except when the peak to continuum ratio exceeds 3.0 , in which case the scale runs from 0.0 to the peak value.

stars, $-58 \AA$. Table 3 summarizes the median statistics, excluding brown dwarfs.

Several environmentally young stars show evidence of a blueshifted absorption component superposed on the $\mathrm{H} \alpha$ emission profile. This feature is often seen in the profiles of accreting TTSs and is usually attributed to absorption from an outflowing jet or wind (e.g., Edwards et al. 1987; Alencar \& Basri 2000).

The full widths at $10 \%$ of the peak flux levels (hereafter called $10 \%$ widths) of the $\mathrm{H} \alpha$ profiles are listed in Table 4.
This profile measurement has been proposed as a useful diagnostic of accretion (e.g., White \& Basri 2003). For the environmentally young star sample, these values range from 139 to $610 \mathrm{~km} \mathrm{~s}^{-1}$. The majority of the $\mathrm{H} \alpha$ profiles are broad ( 25 of 28 have $10 \%$ widths $\gtrsim 250 \mathrm{~km} \mathrm{~s}^{-1}$ ), consistent with the predictions of a magnetically channeled accretion flow (e.g., Hartmann et al. 1994). Nevertheless, several optically veiled and presumably accreting stars show relatively narrow $10 \%$ widths (e.g., IRAS $04260+2642$ and HK Tau B). The distribution of $\mathrm{H} \alpha 10 \%$ widths of class I stars is marginally different 

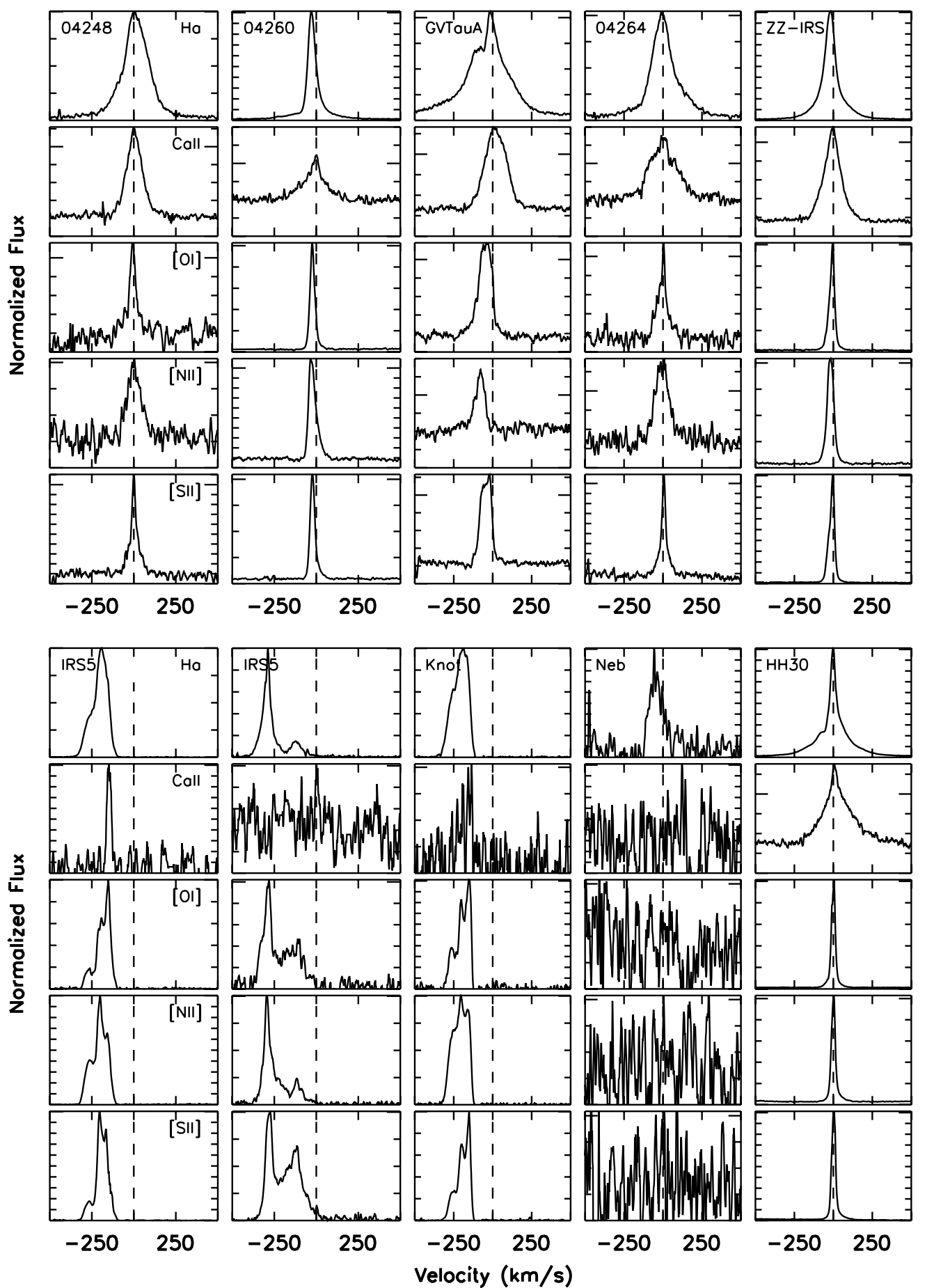

FIG. 7.-Continued

from that of non-HH stars (K-S probability $=0.062$, a $1.9 \sigma$ difference); class I stars have narrower $10 \%$ widths, in the median, than class II star $\left(356 \mathrm{~km} \mathrm{~s}^{-1}\right.$ vs. $\left.429 \mathrm{~km} \mathrm{~s}^{-1}\right)$. In contrast, distributions of $10 \%$ widths for $\mathrm{HH}$ stars and non-HH stars are indistinguishable (K-S probability $=0.77)$, and the median values are essentially the same (410 and $413 \mathrm{~km} \mathrm{~s}^{-1}$, respectively). Table 3 lists the median values, excluding brown dwarfs.

\subsubsection{Forbidden-Line Emission}

The optically thin forbidden emission lines are believed to originate in an outflowing jet or wind. Their intensity is therefore expected to be directly proportional to the amount of material being funneled along the jet, as viewed through the slit of the spectrograph. The strongest forbidden emission lines in our spectra are [O I] $\lambda 6364$ and [S II] $\lambda 6731$, both of which are detected in 21 of the 28 environmentally young stars observed. Figure 9 shows the distributions of EW[S II]s versus $T_{\text {bol }}$ and $\alpha$. No lower limits are plotted, but upper limits are. Additional EW measurements of Taurus $T$ Tauri stars are included using the values measured by Hartigan et al. (1995, all velocities).

The environmentally young stars have EW[S II] within the range of values measured for environmentally older stars, but the distributions are different. The median EW[S II] of class I 


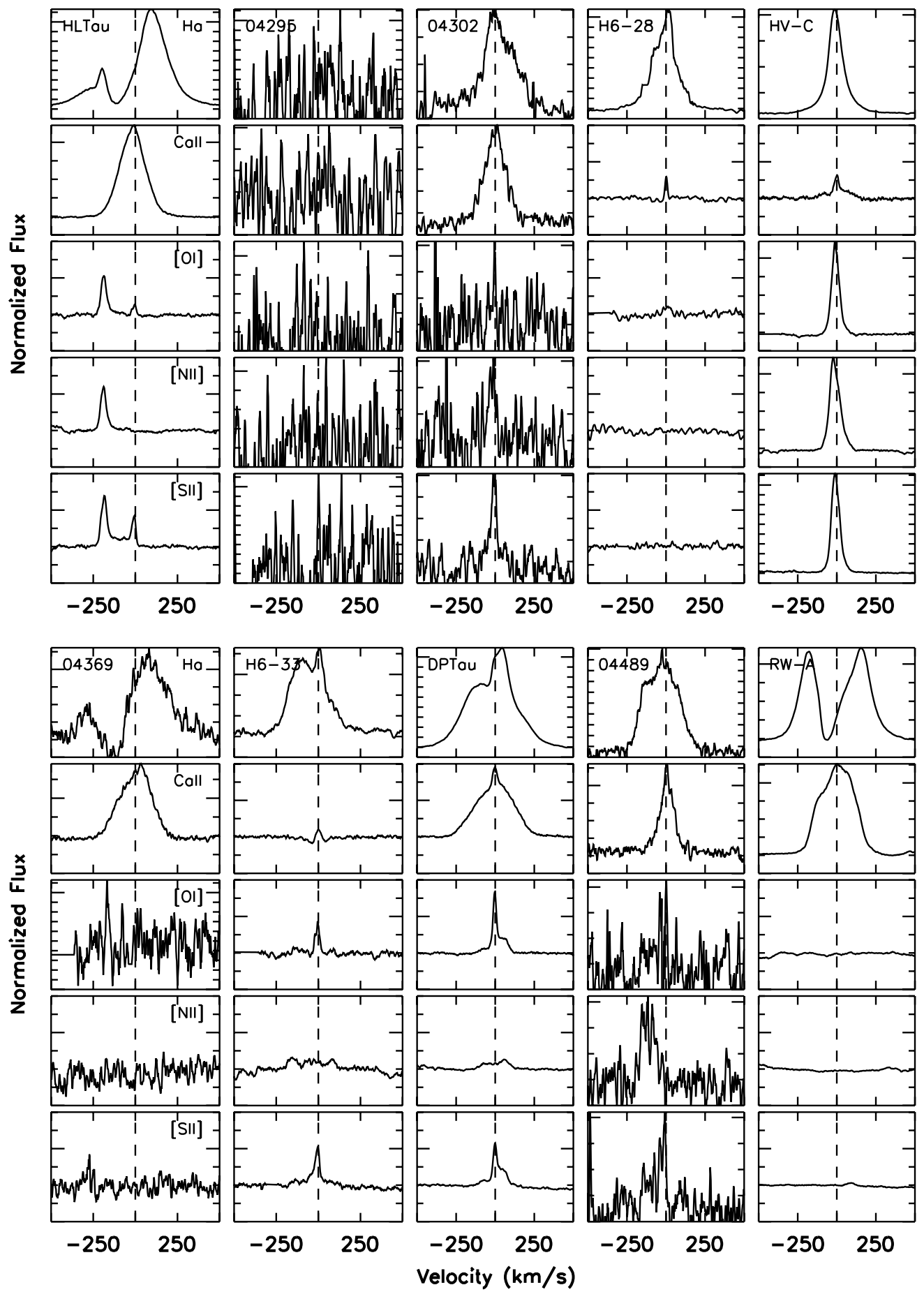

FIG. 7.-Continued

stars is larger than that of class II stars (median class I EW = $-3.1 \AA$; median class II EW $=-0.15 \AA$ ), and the distributions are statistically different $\left(\mathrm{K}-\mathrm{S}\right.$ probability of $\left.1.9 \times 10^{-5}\right)$. Similarly, the median EW[S II] of HH stars is larger than that of non-HH stars (median $\mathrm{HH} \mathrm{EW}=-1.8 \AA$; median non-HH $\mathrm{EW}=-0.14 \AA$ ), and the distributions are different, though less significantly (K-S probability of 0.0086 ). Table 3 lists the median values, excluding brown dwarfs. These comparisons may be somewhat biased by the large fraction of [S II] nondetections (31\% for class I stars, 34\% for class II stars, $15 \%$ for $\mathrm{HH}$ stars, and $47 \%$ for non-HH stars), which are treated as detections. Nevertheless, comparing only systems with detected emission, the EW[S II]s of environmentally young stars are still systematically larger than those of environmentally older T Tauri stars.

The forbidden emission lines of $\mathrm{T}$ Tauri stars are often blueshifted and occasionally show double-peaked profiles, with one peak near the stellar velocity and the second offset blueward by $50-300 \mathrm{~km} \mathrm{~s}^{-1}$ (e.g., Appenzeller et al. 1984; Mundt et al. 1987; Edwards et al. 1987). Only three of the 21 environmentally young stars with forbidden-line emission show a distinct double-peaked emission profile (DG Tau B, 

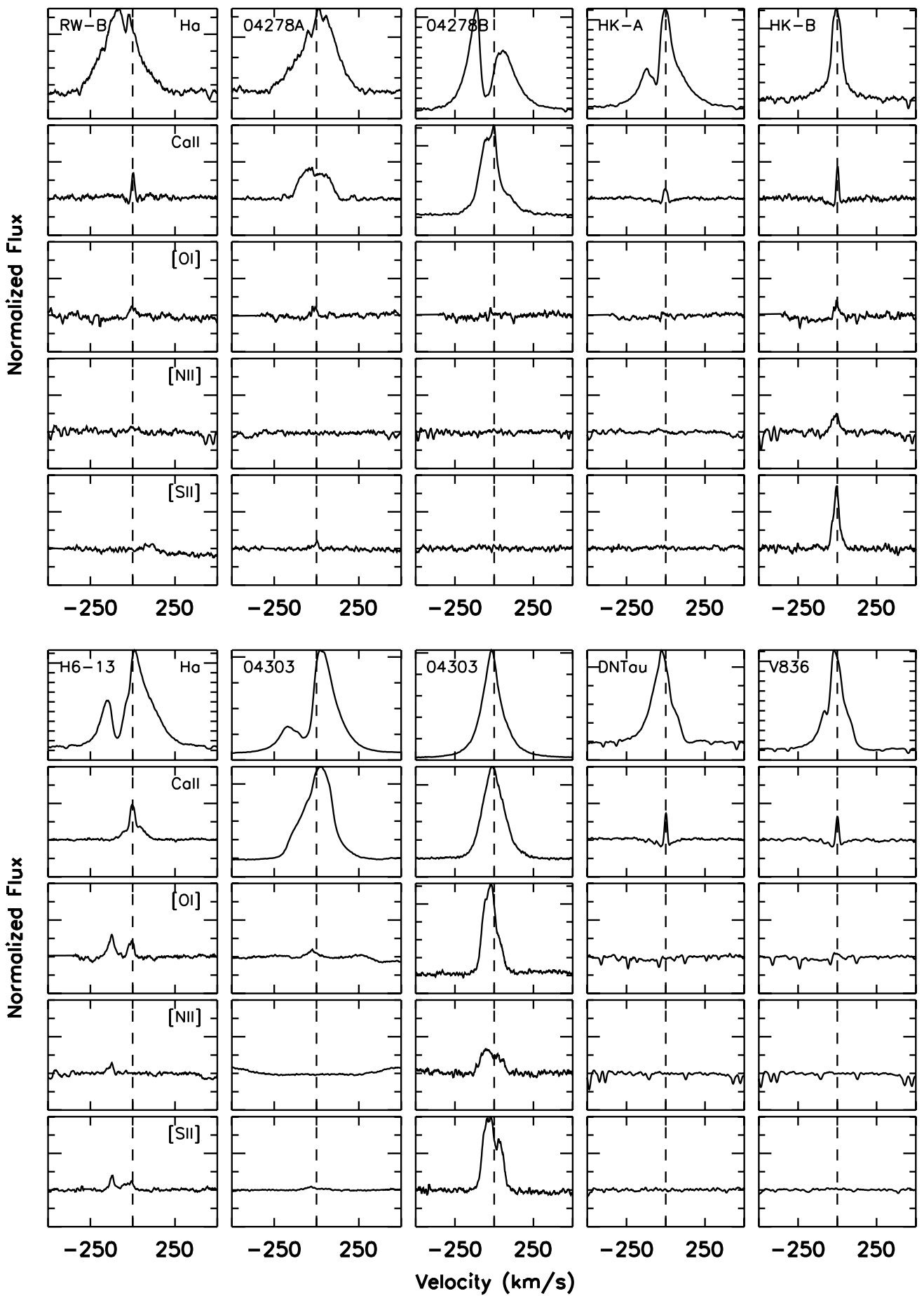

FIG. 7.-Continued

L1551 IRS 5, and HL Tau). One of the additional TTS stars observed also shows a double peak (Haro 6-13). In the two cases for which the systemic velocity is known (HL Tau and Haro 6-13), the blueshifted peaks are offset by approximately -200 and $-100 \mathrm{~km} \mathrm{~s}^{-1}$, respectively. About one-third of the 21 environmentally young stars with [O I] emission have forbidden emission peaks close to the systemic velocity, but with wings extending blueward by $\gtrsim 50 \mathrm{~km} \mathrm{~s}^{-1}$. Half of the stars observed, however, show only forbidden emission at or very near the systemic velocity. This fraction is slightly higher if only class I stars are considered. Of the 13 class I stars with [O I] emission, only five show evidence of a blueshifted component (MHO-2, GV Tau A, L1551 IRS 5, HL Tau, and IRAS $04489+3042)$. This effect could be explained if the jet emission in the majority of class I stars observed propagates perpendicular to our line of sight.

3.1.4.3. The Effect of Orientation on the Emission-Line Profiles

The orientation of each star/disk/jet system relative to our line of sight may have effects on both the measured EWs and emission-line profiles. One of the most useful qualities of an EW is that, by definition, it offers a direct measure of the 

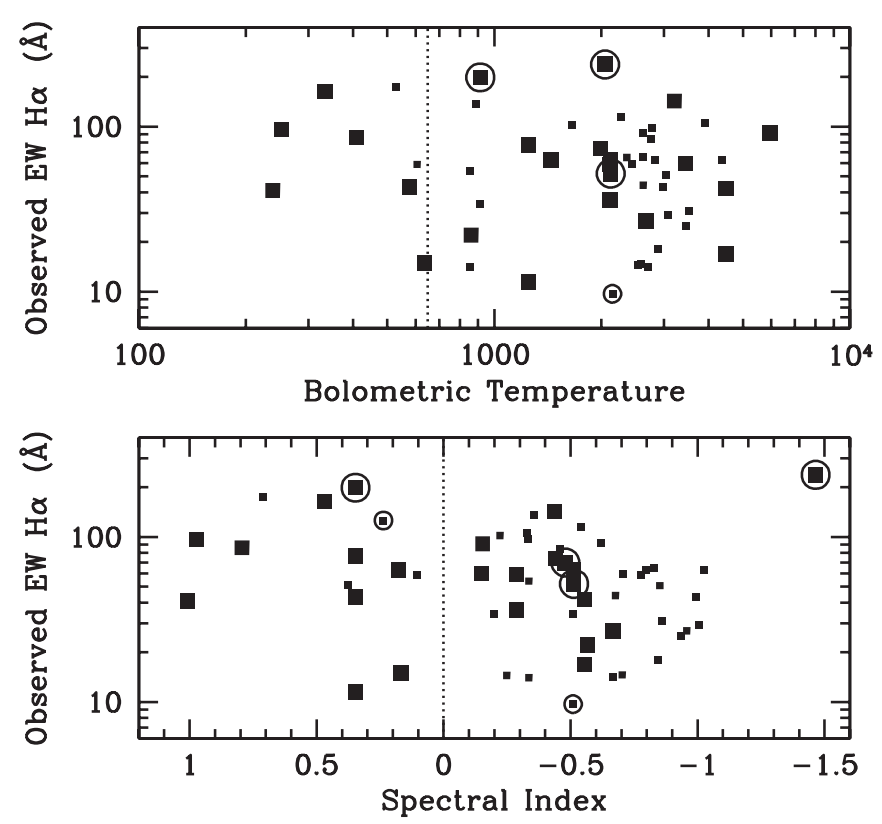

FIG. 8.-Observed EW[H $\alpha]$ vs. bolometric temperature (top panel) and spectral index (bottom panel). The symbols are the same as in Fig. 4; circled symbols indicate stars with known or likely edge-on disk systems (see $\S 3.1 .4$ ). The EW $[\mathrm{H} \alpha]$ does not evolve significantly with either evolutionary diagnostic. $\mathrm{HH}$ stars and non-HH stars have similar $\mathrm{EW}[\mathrm{H} \alpha]$ values.

emission-line flux relative to the continuum, independent of extinction. Thus, if the spectra are not flux calibrated and the extinction is not accurately known, as is the typical case here, the total emission-line flux may still be determined if the intrinsic flux from the stellar continuum can be estimated (e.g., by assuming a flux density distribution based on age and distance). However, this assumption is only valid if the continuum and emission-line fluxes originate from the same region and thus have the same extinction. This is most likely true for the permitted emission lines of young stars, which in general appear to be optically thick and originate in high-density regions close to the star. However, this assumption may not be true for the forbidden emission lines, which often originate in regions that are spatially extended from the star (e.g., Reipurth 1999). In such a case, the emission-line region may be more directly observable than the young partially embedded central star is. The preferentially attenuated continuum flux will consequently produce artificially large EW values.

The three systems with well-defined edge-on disks based on direct imaging, HH 30, HV Tau C, and HK Tau B, likely suffer from this bias to some degree. The forbidden emission line profiles of the edge-on disk systems are narrow and centered on the systemic velocity, consistent with a bipolar flow perpendicular to our line of sight. High spatial resolution Hubble Space Telescope images of both HH 30 and HV Tau C show spatially extended [ $\left.\mathrm{O}_{\mathrm{I}}\right]$ and [S II] emission (Burrows et al. 1996; Bacciotti et al. 1999; Stapelfeldt et al. 2003); this emission is more directly observable than the continuum stellar emission that is scattered into our line of sight. Preferential attenuation of the continuum likely explains why the EWs of [S II] $\lambda 6731$ for HH 30 and HV Tau C are more that 2 orders of magnitude larger than those of any Taurus $T$ Tauri star in the survey of Hartigan et al. (1995). The possibly biased EWs of edge-on disk systems are therefore distinguished in Figure 9. A preferentially attenuated continuum may also explain the large forbidden emission line EW lower limits of the two stars with no detected continuum (DG Tau B and L1551 IRS5). Without spatial information on the origin of these emission-line features and the efficiency of scattered continuum emission, this is not something we can correct for. We highlight this effect, however, as a potential bias of the forbidden emission line EWs and the mass outflow rates inferred from them $(\S 3.3 .2)$.

In contrast to the forbidden emission line EWs, the $\mathrm{H} \alpha$ EWs of the three edge-on type disk systems are consistent with the EW distribution of the larger, presumably randomly oriented sample. This agrees with the expectation that the majority of the $\mathrm{H} \alpha$ emission originates very close to the star and therefore experiences the same attenuation as the stellar continuum. However, they nevertheless have relatively narrow $\mathrm{H} \alpha 10 \%$ widths $\left(293,261\right.$, and $\left.196 \mathrm{~km} \mathrm{~s}^{-1}\right)$. On average, these values are more consistent with those of nonaccreting T Tauri stars, with typical $10 \%$ widths less than $270 \mathrm{~km} \mathrm{~s}^{-1}$ (White \& Basri 2003). Assuming that the accretion occurs via the same magnetically channeled high-velocity flow for these three stars, then either the edge-on orientation prevents a direct view of the highvelocity emission or the projected velocities along our line of sight are low. The absence of a strong blueshifted absorption component superposed on the $\mathrm{H} \alpha$ emission is also consistent with an outflow perpendicular to our line of sight.

In light of the emission-line properties of the known edge-on disk systems HH 30, HV Tau C, and HK Tau B, we suggest that three additional stars with similar properties, CoKu Tau 1, IRAS $04260+2642$, and ZZ Tau IRS, may also have nearly edge-on orientations. This prediction is based on (1) the relatively narrow $\mathrm{H} \alpha 10 \%$ widths $\left(285,139\right.$, and $\left.268 \mathrm{~km} \mathrm{~s}^{-1}\right)$, (2) the absence of any superposed absorption on the $\mathrm{H} \alpha$ emission profiles, and (3) the unusually large strengths of their forbidden emission lines, which are both narrow and very close to the systemic velocity (see Fig. 7 and Table 3 ). The emission lines of IRAS $04158+2805$ and IRAS $04248+2612$ are also marginal, consistent with an edge-on orientation, but the narrow profiles of these two stars may simply result from the lower infall/outflow velocities expected in substellar objects (e.g., Jayawardhana et al. 2003). In a few of these cases, however, we note that the narrow forbidden emission lines are centered $\sim 10-40 \mathrm{~km} \mathrm{~s}^{-1}$ blueward of the systemic velocity (e.g., IRAS 04158+2805, IRAS 04260+2642, and ZZ Tau IRS), suggesting that these systems are close to, but not completely edge-on.

\subsubsection{Emission-Line Variability}

L1551 IRS 5 and IRAS 04303+2240 show significant changes in emission-line properties over a timescale of $\sim 3 \mathrm{yr}$. During the first epoch observations of IRAS $04303+2240$, when the star is much more optically veiled and presumably accreting at a higher rate, the permitted emission lines $(\mathrm{H} \alpha$, $\mathrm{Ca}$ II) show evidence of absorption from a blueshifted outflow, but the forbidden emission lines are very weak or absent. During the second epoch, when the star is less optically veiled, the permitted line profiles are slightly narrower, more symmetric, and show no evidence a superposed absorption. The forbidden emission lines, however, are considerably stronger. Even after accounting for the diminished continuum excess, the EW fluxes of [ $\left.\mathrm{O}_{\mathrm{I}}\right]$, [ $\mathrm{N}$ II], and [S II] are all 1 order of magnitude larger during the second epoch.

No continuum emission is detected in the first observation of L1551 IRS 5, but it is marginally detected in the second ( $\mathrm{S} / \mathrm{N}=1.4$ at $8450 \AA$ ). Like IRAS $04303+2240$, the emissionline profiles of L1551 IRS 5 changed significantly over the same 3 yr timescale. The changes included broader emission profiles for both $\mathrm{H} \alpha$ and the forbidden emission lines, the 
TABLE 4

Permitted and Forbidden Emission-Line Properties

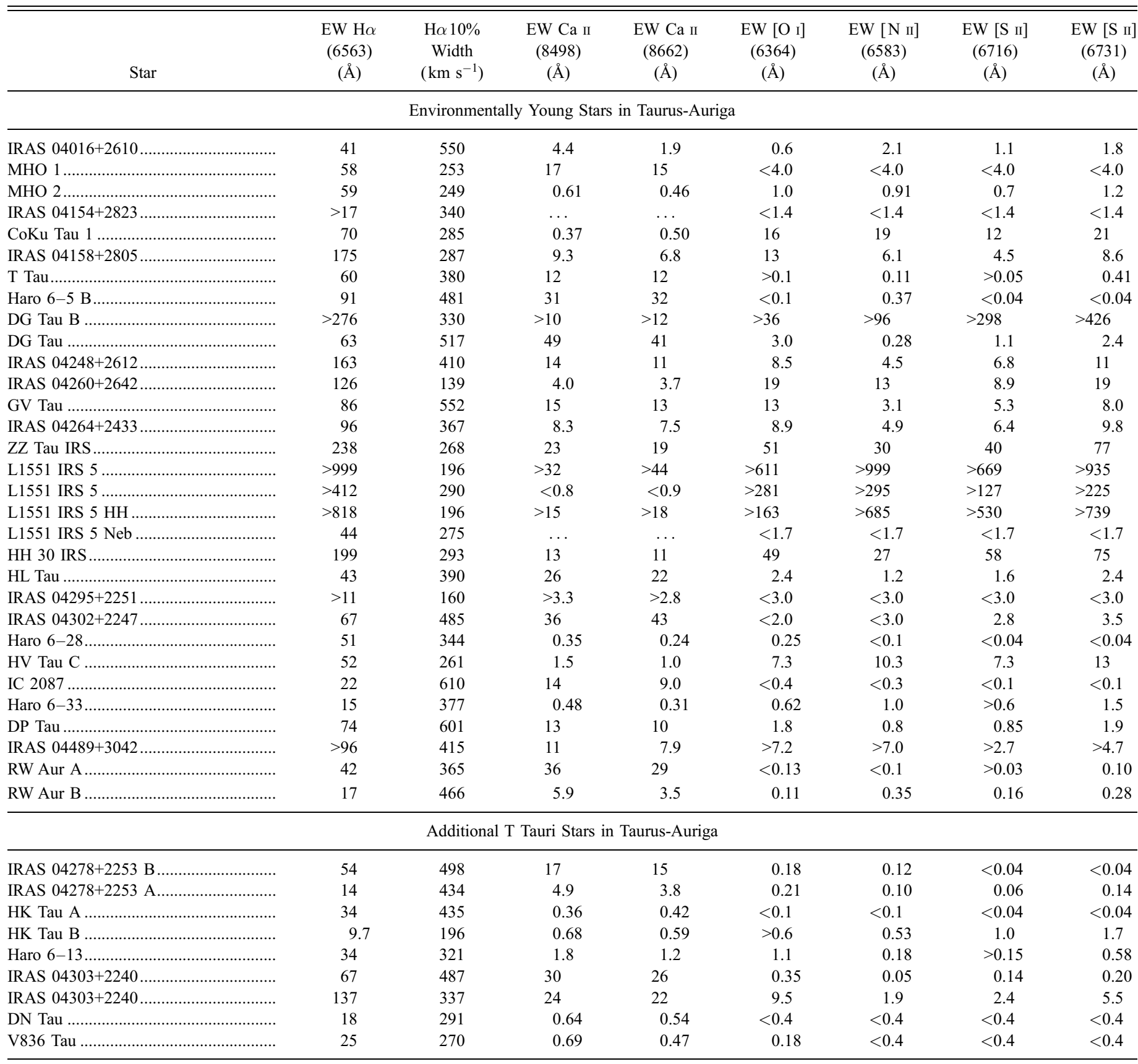

disappearance of the Ca II emission, and possibly weaker line emission overall (upper limits are lower). It is interesting that the emission-line profiles of the first epoch of IRS 5 are quite similar to the emission-line profiles of the HH knot obtained during the second epoch. The similarities include both the approximate velocity profile of the $\mathrm{H} \alpha$ emission and the triplepeaked profiles of the forbidden-line emission.

The spectrum of the L1551 nebula is the only spectrum from this system that shows $\mathrm{H} \alpha$ emission with a peak close to the expected systemic velocity (i.e., the mean of Taurus). This may actually be scattered emission from the star. Low-resolution spectra of this nebula have identified stellar absorption features of a G-type photosphere (Mundt et al. 1985). No photospheric features were detected in our spectrum. Observations of this scattered light nebula may be the only way to ob- served the stellar emission from L1551 IRS 5 at optical wavelengths.

\subsection{Inferred Stellar Properties}

\subsubsection{Stellar and Bolometric Luminosities}

One of the main motivations for determining the stellar luminosities of the stars identified as environmentally young in Table 1 is to see whether they are in fact younger than more optically revealed T Tauri stars. Since low-mass stars contract primarily along Hayashi tracks, maintaining roughly constant temperature, younger stars should have larger radii and be more luminous. The luminosity of a young star is typically calculated from a reddening-corrected photometric measurement (e.g., $I_{c}$ or $J$ ) and a bolometric correction corresponding 

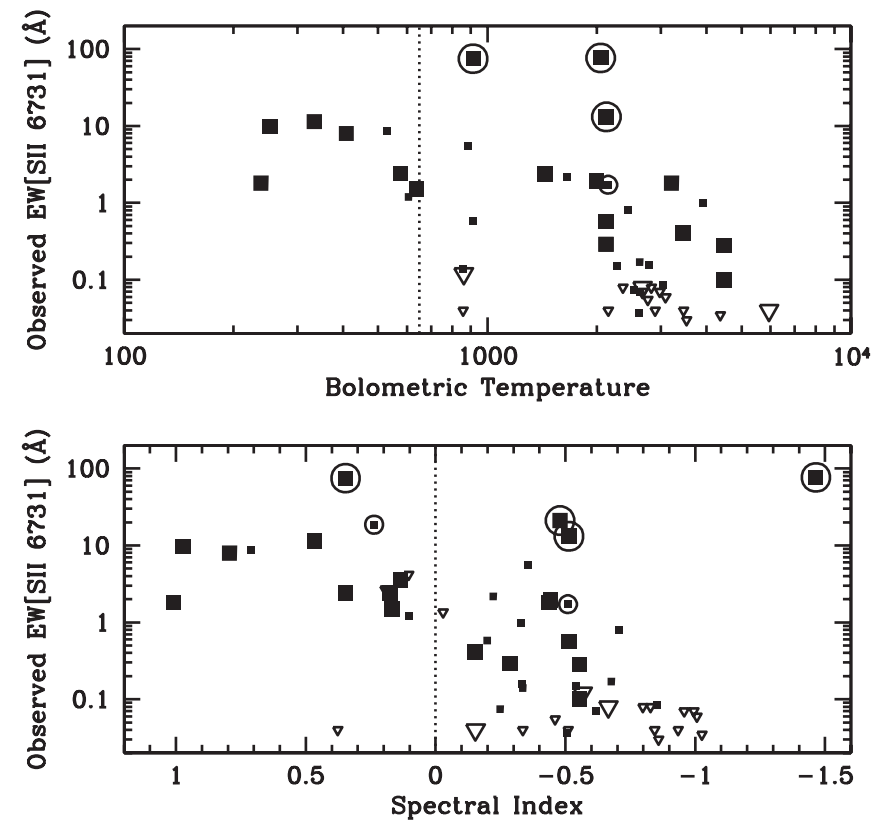

FIG. 9.-Observed EW [S II 26731$]$ emission vs. bolometric temperature (top panel) and spectral index (bottom panel). The dashed lines and symbols are the same as in Fig. 4; circled symbols indicate stars with known or likely edge-on disk systems. The average EW[S II] decreases toward hotter bolometric temperatures and negative spectral indices. HH stars have systematically larger EW[S II]s than non-HH stars. Stars with edge-on orientation have $\mathrm{EW}[\mathrm{S}$ II] $\mathrm{s}$ biased toward artificially large values (§ 3.1.4).

to its spectral type. Although most of the stars observed here have both measured photometry and now a spectral type, ascertaining the intrinsic stellar energy distribution and the proper reddening correction necessary to estimate their stellar luminosities is inhibited by the extensive nonphotospheric emission associated with these stars. At optical wavelengths, scattered light emission can increase the apparent stellar flux measured in a fixed aperture and can cause underestimates of extinction because of preferential blue scattering. At near-infrared wavelengths, the general trend of increasing thermal emission from the disk toward longer wavelengths can lead to overestimates of the extinction. Despite these possible systematic uncertainties, we explore several traditional means for calculating stellar luminosities. We consider dereddening the stars using colors that are least uncontaminated, that is, as close to photospheric as possible, namely, $I_{c}-J, J-H$, and $H-K_{s} . I_{c}$ magnitudes are listed in Table $1 ; J, H$, and $K_{s}$ magnitudes are from the 2MASS database. The underlying photospheric colors are adopted according to our spectral types and a standard interstellar reddening law (Cohen et al. 1981). For comparison purposes, we apply bolometric corrections to three dereddened magnitudes: $I_{c}, J$, and $K_{s}$. For the fraction of our sample to which all three methods can be applied, comparison of the results reveals systematic effects. Luminosities calculated from $K_{s}$ and $H-K$ are systematically higher than those computed from $J$ and $J-H$ (by $0.35 \mathrm{dex}$ ) or from $I_{c}$ and $I_{c}-J(0.45 \mathrm{dex})$. This is likely due to the systematically larger excess emission at $K_{s}$ caused by thermal emission from hot dust and gas. However, the differences in luminosities calculated from $J, J-H$ and $I_{c}, I_{c}-J$ are scattered about unity though with large dispersion. This suggests that either may provide an adequate measure of the stellar luminosities. Since we have $J$ and $H$ magnitudes for the majority of stars, we adopt the stellar luminosities calculated from $J-H$ extinctions and a bolometric correction to the $J$ band.
The calculated visual extinction and stellar luminosities are listed in the fourth and fifth columns of Table 5. The uncertainties in these estimates are dominated by systematic effects, which lead to errors of 1-2 mag in $A_{V}$ and factors of 2-3 in $L_{\text {star}}$, with even larger errors for edge-on disk systems (e.g., HH 30).

The average visual extinction of the 11 class I stars with stellar spectral types is $10.5 \mathrm{mag}$, with a standard deviation $4.2 \mathrm{mag}$. This is only a factor of a few larger than typical values for class II stars in Taurus and is a factor of a few less than is often suggested by the rising energy distributions (Greene \& Lada 1996) and scattered light images (e.g., Kenyon et al. 1993a; Whitney et al. 1997) of most class I stars. As described in $\S 2.1$, our class I sample is biased toward those that are more optically revealed.

Figure 10 shows the distribution of stellar luminosities for environmentally young stars in Taurus as a function of effective temperature. The temperatures are estimated from the new spectral types listed in Table 2, assuming the dwarf temperature scale adopted by Hillenbrand \& White (2004). The six environmentally young stars that are likely edge-on disk systems (§ 3.1.4) are marked, since this orientation likely leads to underestimates of their stellar luminosity. The three panels separate stars with $T_{\text {bol }} \leq 650$, stars with $\alpha>0.0$, and stars that power $\mathrm{HH}$ flows. Also shown for comparison are the mean stellar luminosities of class II and class III T Tauri stars in Taurus (environmentally young stars are excluded) measured in six temperature bins. The T Tauri stellar luminosities are taken from Kenyon \& Hartmann (1995) and are determined from a bolometric correction to the $J$ band, as was done here. ${ }^{2}$ Figure 10 also shows the pre-main-sequence evolutionary models of Siess et al. (2000). In comparison with these models, $\mathrm{T}$ Tauri stars in Taurus have a mean age of a few times $10^{6} \mathrm{yr}$, but with systematically younger ages $\left(<10^{6} \mathrm{yr}\right)$ at the lowest masses and larger ages $\left(\sim 10^{7} \mathrm{yr}\right)$ at the highest masses. The apparent mass-dependent age inferred here is often seen in comparisons of cluster isochrones with pre-main-sequence evolutionary models and may be caused by incorrect assumptions in evolutionary models or the assumed temperature scale. Finally, the temperatures and luminosities corresponding to the "stellar birth line" for spherical accretion (Stahler 1988) are shown, using the mass-radius relationship defined in Fletcher \& Stahler (1994) as applied to the Siess et al. (2000) evolutionary models.

The stellar luminosity estimates of the environmentally young stars are consistent, on average, with the mean T Tauri star luminosities. At masses below $\sim 1 M_{\odot}$, the stellar birth line is systematically overluminous by $0.3-0.5$ dex compared to the mean T Tauri luminosities and the environmentally young star luminosities, though statistically consistent with both of these distributions. At masses above $\sim 1 M_{\odot}$, however, the stellar birth line is systematically larger by $0.5-1.0 \mathrm{dex}$ than the mean $\mathrm{T}$ Tauri luminosities and only marginally consistent with the environmentally young luminosities. We note that the $10^{6} \mathrm{yr}$ isochrone reasonably matches the mean luminosities of environmentally young stars over all masses. For direct comparison (and further use in $\S 3.3$ below), we list the $10^{6}$ yr luminosity corresponding to the stellar temperature for all stars with

2 The mean T Tauri luminosities do not include the stars HBC 358, 359, $360,361,362,372$, and 392 , which have insufficient lithium to be confidently considered T Tauri stars, and do not include the binaries HBC 354/355 or HBC 356/357, whose primaries lie below the main sequence assuming a distance of $140 \mathrm{pc}$. 
TABLE 5

Inferred Stellar, Accretion, and Outflow Properties

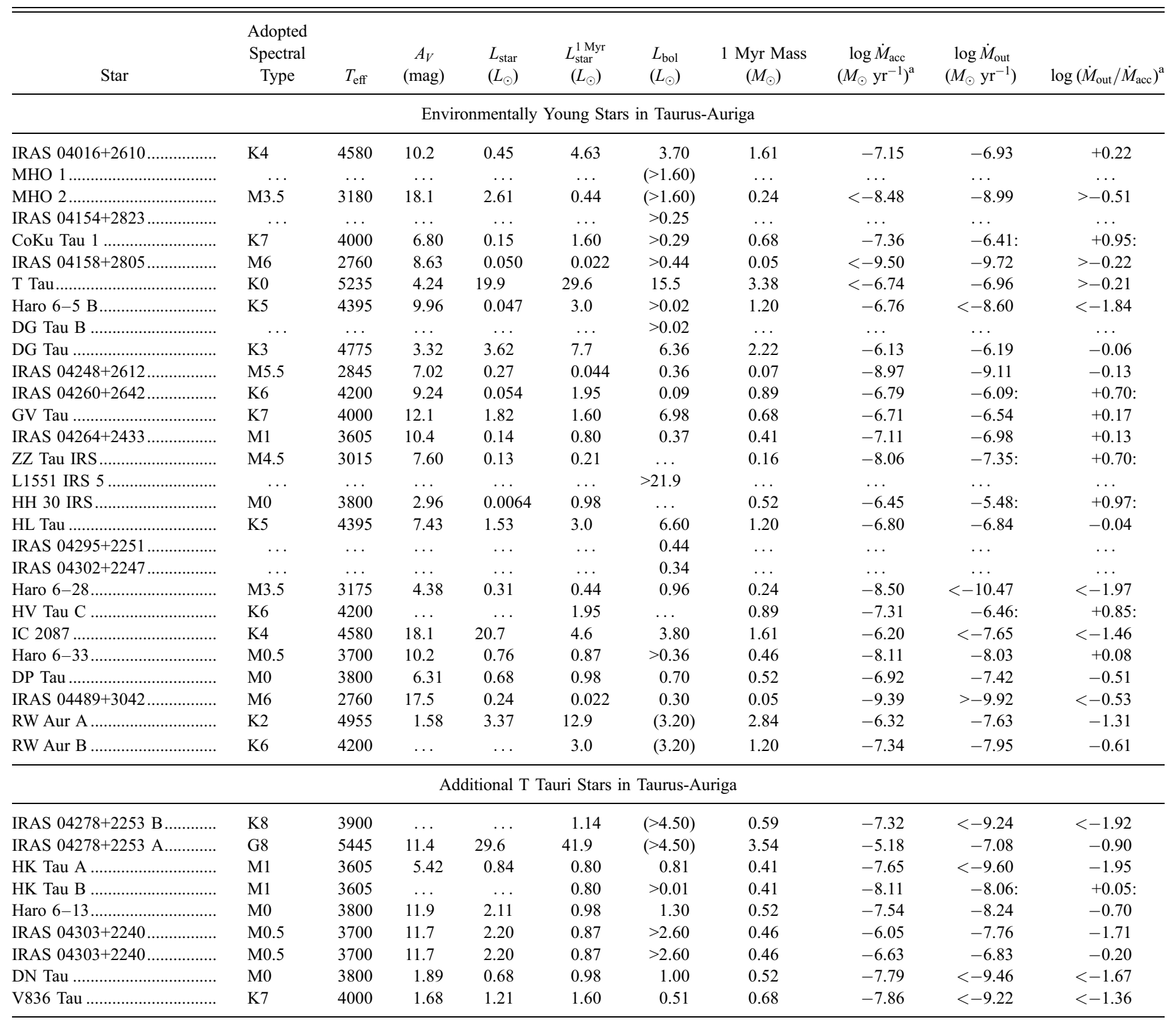

${ }^{a}$ Values marked with a colon indicate edge-on disk systems. The [S II] emission in these systems appears to be biased toward large values, leading to overestimates of $\dot{M}_{\text {out }}$ and $\log \left(\dot{M}_{\text {out }} / \dot{M}_{\text {acc }}\right)$.

temperature estimates in Table $4\left(L_{\mathrm{star}}^{1 \mathrm{Myr}}\right)$. Independent of selection criteria, environmentally young stars appear to be coeval with populations of class II and III stars in Taurus.

\subsubsection{Stellar Masses and Angular Momentum}

The stellar luminosity and temperature estimates of the environmentally young stars allow direct comparison with premain-sequence evolutionary models to estimate stellar masses. However, we do not favor computing masses via direct comparison given the large spread in stellar luminosities, likely caused by biases introduced from circumstellar material. The cool temperatures of these $\mathrm{K}$ and $\mathrm{M}$ type young stars suggest that most should reside on the convective and predominantly vertical (constant temperature) portion of their pre-mainsequence evolution. Their temperature principally will determine their stellar mass. Therefore, we use the temperature-mass relation defined by the Siess et al. (2000) 1 Myr isochrone to estimate masses for the stars in our sample with spectral type estimates. As noted above, the $10^{6} \mathrm{yr}$ isochrone reasonably represents the average apparent age of these stars. Masses for objects cooler than the lowest mass track $\left(0.1 M_{\odot}\right)$ are estimated to be $0.07 M_{\odot}$ at spectral type M5.5 and $0.05 M_{\odot}$ at spectral type M6, based on both a modest extrapolation of the Siess et al. (2000) models and comparisons with other evolutionary models (e.g., Baraffe et al. 1998).

The inferred stellar/substellar masses are listed in Table 3 and span nearly 2 orders of magnitude: $0.05-3.5 M_{\odot}$. The distribution of masses is shown in Figure 11 as a function of the two evolutionary diagnostics $T_{\mathrm{bol}}$ and $\alpha$. For comparison, the additional sample of class II stars is shown; their masses are determined from their assigned spectral types and the same temperature-mass relationship used above. The similar spectral 


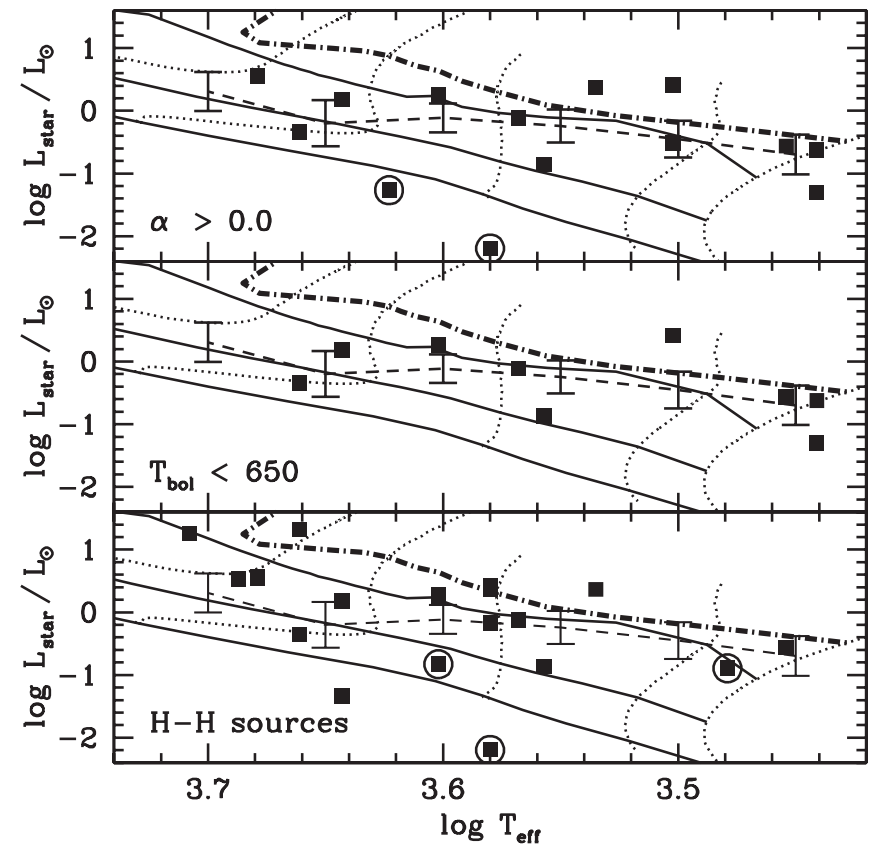

FIG. 10.- H-R diagrams for the young stars in Taurus selected according to three evolutionary diagnostics: spectral index greater than $0.0, T_{\text {bol }} \leq 650$, and stars that power $\mathrm{HH}$ flows. Values are from Table 3 ; circled squares indicate stars with known or likely edge-on disk systems. Typical uncertainties are 0.4 in $\log L$ and 0.02 in $\log T$. The dashed line in each plot indicates the mean stellar luminosities of optically visible Taurus $\mathrm{T}$ Tauri stars, and error bars indicate the dispersion in these luminosities. The evolutionary models of Siess et al. (2000) are shown with isochrones at $10^{6}, 10^{7}, 10^{8} \mathrm{yr}$ (solid lines), and mass tracks at $0.2,0.5,1.0,2.0$, and $4.0 M_{\odot}$ (dotted lines). The thick dot-dashed line indicates the "stellar birth line" for the case of $\dot{M}_{\mathrm{acc}}=10^{-5} M_{\odot} \mathrm{yr}^{-1}$ (Fletcher \& Stahler 1994). The environmentally young stars appear to have ages of $\sim 10^{6} \mathrm{yr}$, similar to the mean ages of environmentally older Taurus $\mathrm{T}$ Tauri stars.

type distribution of class I and class II stars $(\S 3.1 .2)$ translates into a similar mass distribution. Likewise, the slight trend toward hotter spectral types for $\mathrm{HH}$ stars translates into a slight difference in the stellar mass distributions; HH stars are slightly more massive. It should be noted, however, that the comparison class II population shown in Figure 11 is not representative of the true distribution of stellar (and substellar) masses in Taurus. The now well-established populations of low-mass stars and brown dwarfs in Taurus (e.g., Briceño et al. 1998, 2002; Martín et al. 2001; Luhman et al. 2003) are in general too faint to have been detected in previous mid- and far-infrared surveys (e.g., $I R A S$ ), from which spectral indices and bolometric temperatures can be determined. Thus, without these evolutionary diagnostic measurements, they cannot be plotted in Figure 11. Nevertheless, the three class I stars IRAS $04158+2805$, IRAS $04248+2612$, and IRAS $04489+3042$ are of particular interest. With spectral types of M5.5 or cooler, they have substellar masses and are the first spectroscopically confirmed class I brown dwarfs.

Figure 12 shows the $v \sin i$ values measured in $\S 3.1 .1$ normalized by their breakup velocity, defined as $v_{\mathrm{br}}=(G M / R)^{1 / 2}$. Stellar mass and radius estimates are from the adopted Siess et al. (2000) $10^{6} \mathrm{yr}$ isochrone described above. The normalized velocities range from less than $5 \%$ to $20 \%$ of the breakup velocities. K-S comparisons of class I stars with class II stars and of $\mathrm{HH}$ stars with non-HH stars show no differences in distributions (K-S probabilities of 0.079 and 0.096 , respectively). The difference in the $v \sin i$ distributions of $\mathrm{HH}$ stars and non-HH stars ( $\S 3.1 .1$ ) disappears when these values are nor-
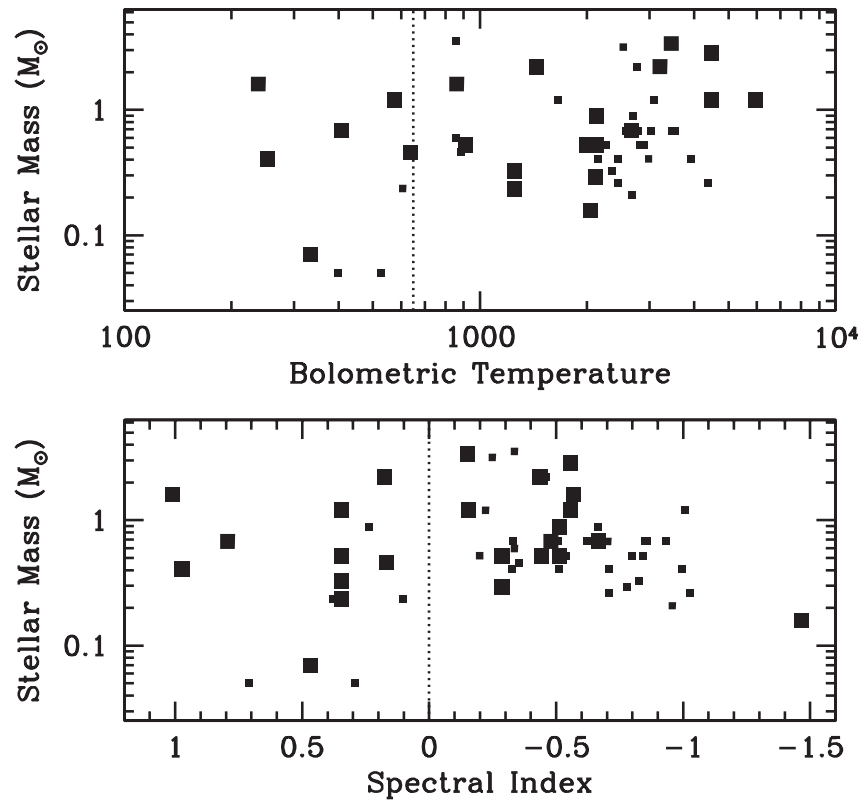

FIG. 11.- Stellar mass vs. bolometric temperature (top panel) and spectral index (bottom panel). The symbols are the same as in Fig. 4. class I stars have masses similar to class II stars, ranging from substellar masses to several solar masses. Note that although many class II brown dwarfs are known, they are not plotted here, since they do not have bolometric temperature or spectral index values because of insufficient infrared and submillimeter measurements.

malized by the breakup velocity, possibly because of the slight mass difference between $\mathrm{HH}$ and non-HH stars. There is no evidence for a change in the angular momentum between the environmentally young and the T Tauri samples.

\subsection{Inferred Circumstellar Properties}

In this section mass accretion rates are estimated from the observed continuum excesses and mass outflow rates are
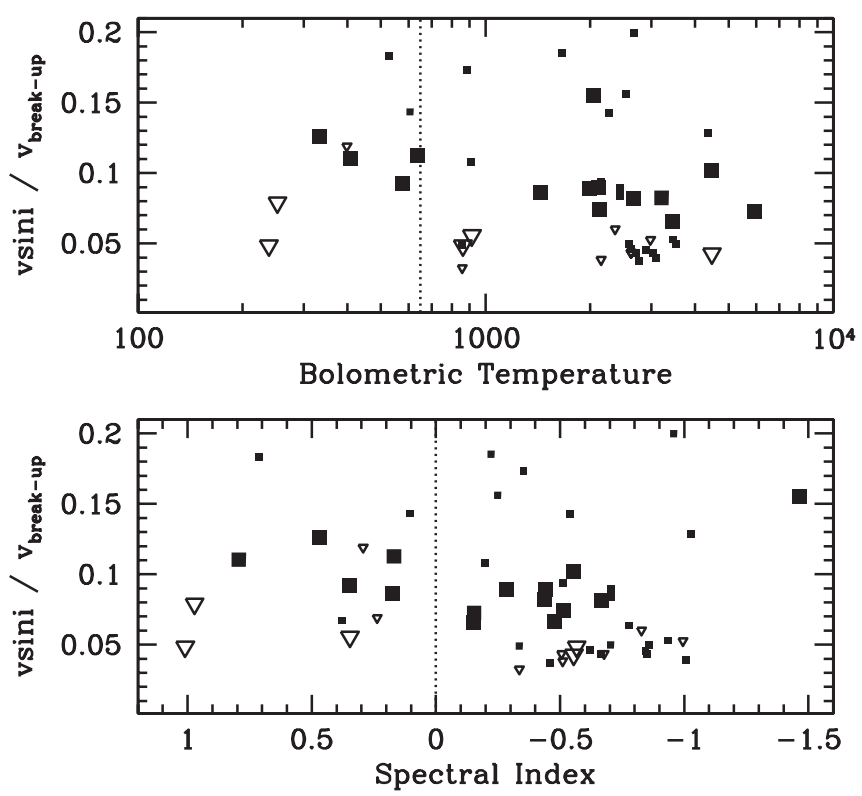

FIG. 12.-Projected rotational velocities $(v \sin i)$ normalized by the breakup velocity vs. bolometric temperature (top panel) and spectral index (bottom panel). The symbols are the same as in Fig. 4. Angular momentum does not evolve significantly with either evolutionary diagnostic. The angular momentum of $\mathrm{HH}$ stars and non-HH stars are similar. 
estimated from the strengths of forbidden-line emission, under the assumption of a bipolar flow. We outline the steps of these procedures in some detail to highlight the substantial uncertainties in current estimates. The ratio of these quantities is used to constrain the origin and energetics of these apparently physically related processes.

\subsubsection{Mass Accretion Rates}

The continuum excesses observed in T Tauri star spectra are attributed to the high-temperature regions generated as accreting material shock decelerates at the stellar surface. Measurements of this excess can therefore be used to estimate the mass accretion rate under the assumption that the liberated energy was gravitational potential energy. The steps involved in estimating mass accretion rates are illustrated in the five panels of Figure 13. Figure 13 contains only a subset of our data; it is restricted to a narrower mass range for direct comparison with the stellar and accretion properties of the 17 Taurus $\mathrm{T}$ Tauri stars studied by Gullbring et al. (1998). The mean and range from the Gullbring et al. (1998) study are indicated in each panel.

The top panel of Figure 13 shows the distribution of continuum excesses at $6500 \AA\left(r_{6500}\right)$ as a function of stellar mass for stars observed here and the additional sample of class II stars ${ }^{3}$ ( 33 ). The distribution of continuum excesses is indistinguishable from that measured by Gullbring et al. (1998) at bluer wavelengths (3200-5300 ̊). As noted by (Basri \& Batalha 1990) and further supported by the results in $\S 3.1 .2$, the veiling is relatively constant redward of $\sim 5000 \AA$.

Since the continuum excess measurements are determined relative to the photosphere $\left(r=F_{\text {ex }} / F_{\text {phot }}\right)$, the photospheric flux needs to be known in order to determine the continuum excess flux. Since current estimates of the photospheric flux are subject to very large uncertainties $(\S 3.2 .1)$, we assign stellar flux values based on the predictions of the Siess et al. (2000) evolutionary models for $1 \mathrm{Myr}$ aged stars as observed through the $R_{c}$ passband. An $R_{c}$ zero point of $2.32 \times 10^{-9}$ ergs $\mathrm{s}^{-1} \mathrm{~cm}^{-1} \AA^{-1}$ (Hayes 1985) is assumed. The second panel of Figure 13 shows the distribution of stellar luminosities corresponding to the adopted photospheric flux levels. We show stellar luminosities, rather than $R_{c}$-band fluxes, for direct comparison with the Gullbring et al. (1998) values. Our luminosities are slightly larger than those determined by Gullbring et al. (1998), but by less than a factor of 2 in the mean. The third panel in Figure 13 shows the distribution of excess fluxes measured over the 6000-6500 $\AA$ wavelength interval. Surprisingly, the distribution is indistinguishable from that measured over the 3200-5300 A interval by Gullbring et al. (1998); standard hot spot models predict that the excess flux should be only one-tenth that seen in the bluer passband (Hartigan \& Kenyon 2003). Up to a factor of 2 of this discrepancy may be caused by larger stellar luminosities, but the observed red excesses are still larger by a factor of $\sim 5$ compared with standard hot spot model predictions.

In order to convert the excess measurements to the total accretion luminosity, a bolometric correction is needed. Based

\footnotetext{
3 The continuum excess measurements for the additional class II stars are from Basri \& Batalha (1990), Hartigan et al. (1995), and White \& Basri (2003). Although the values measured by Basri \& Batalha (1990) and White \& Basri (2003) are determined near $6500 \AA$, as are those newly presented here, the values measured by Hartigan et al. (1995) are determined at a slightly shorter wavelength, $5700 \AA$. However, direct comparison of the veiling values for stars observed by both Hartigan et al. (1995) and Basri \& Batalha (1990) shows no difference (16 star overlap; median $r_{6500}-r_{5700}=+0.03$ ). We use the $r_{5700}$ values as $r_{6500}$ values.
}

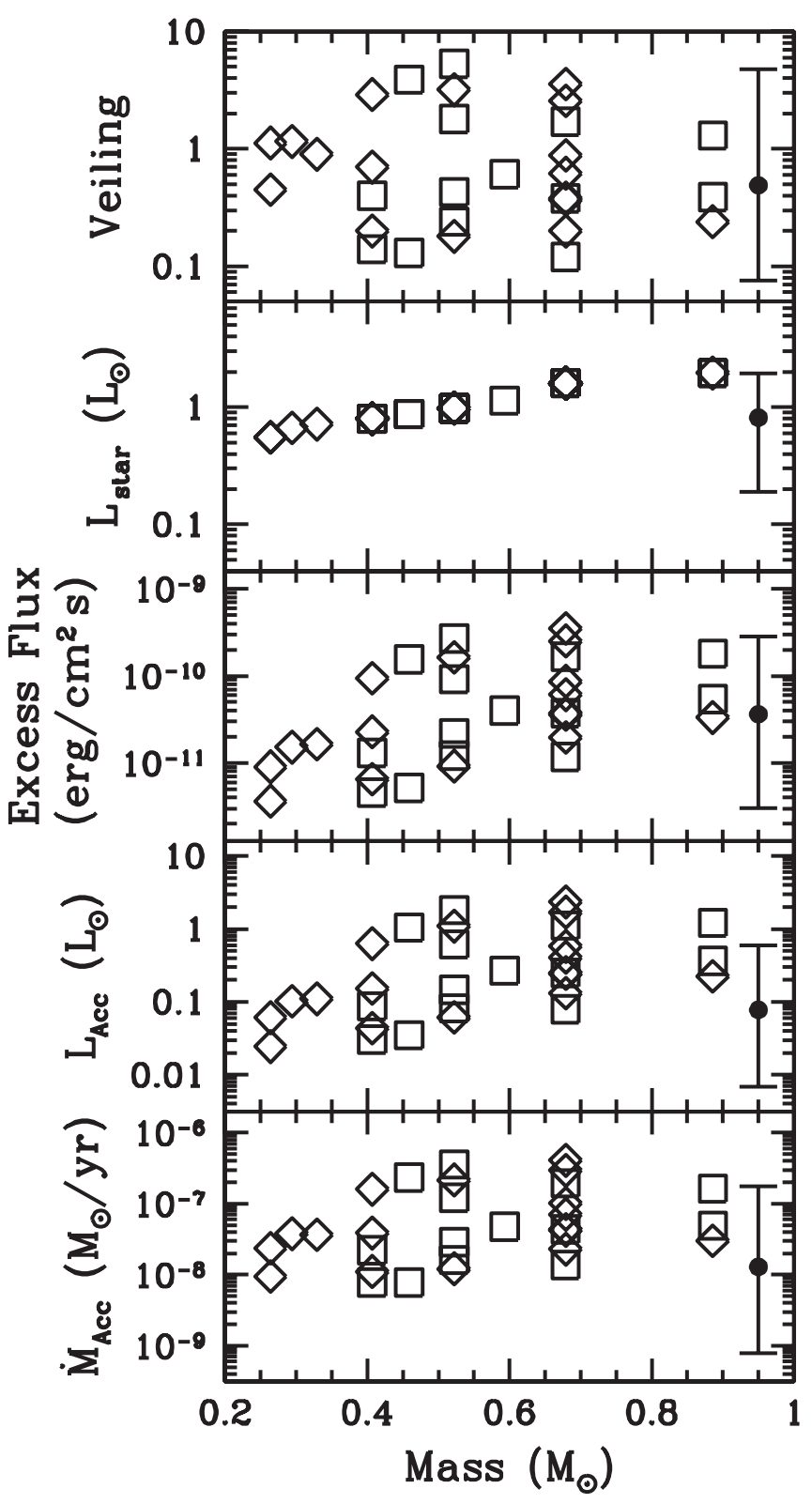

FIG. 13.-Quantities used in the conversion of optical veiling values at $6500 \AA$ to mass accretion rates (veiling, stellar luminosity, excess flux in the 6000-6500 A passband, total accretion luminosity, mass accretion rate) vs. stellar mass. Squares are new veiling measurements and diamonds are veiling measurements from the literature (see $\S 3.3 .1$ ). The vertical error bar in each panel indicates the range of values determined by Gullbring et al. (1998) from a broader but bluer wavelength interval (3200-5300 $\AA$ ), constrained to the same mass range shown here. Surprisingly, the excess flux levels measured over the $6000-6500 \AA$ passband are similar to those measured by Gullbring et al. (1998); the standard hot spot model predicts they should be only onetenth. A factor of 2 can be attributed larger stellar luminosities, from which the excess fluxes are determined relative to, but the red excess fluxes are still a factor of $\sim 5$ greater than predicted. A reduced bolometric correction is adopted to convert the excess flux levels to total accretion luminosity, which leads to accretion luminosities only $\sim 3$ times larger than those of (Gullbring et al. 1998). This, in combination with a different geometric assumption, leads to mass accretion rates that are, on average, $\sim 4$ times larger than those inferred by Gullbring et al. (1998).

on the calculations of Hartigan et al. (1991), who model the emission from the accretion shock as a slab of pure hydrogen gas of constant temperature and density in LTE, Hartigan \& Kenyon (2003) estimate that the bolometric correction for an excess measured over $6000-6500 \AA$ is $\sim 35$. However, this 
bolometric correction, which is 10 times larger than the bolometric correction predicted for the 3200-5300 ̊ passband, will yield accretion luminosities that are 10 times larger than that measured by Gullbring et al. (1998), since the two passbands have similar flux excesses. Consequently, we suspect that the mass accretion rates determined by Hartigan \& Kenyon (2003) are most likely overestimates, as their best-matched model would predict very large blue wavelength excesses, in contrast to what is observed. Similarly, we suspect that inferred mass accretion rates determined by Gullbring et al. (1998) are most likely underestimates, as their best-matched model predicts negligible red excesses, in contrast to what is observed. Without a better model for the total continuum excess flux, we adopt a bolometric correction of 11, corresponding to the logarithm average of the two extremes (3.5 and 35). The resulting accretion luminosities are shown in the fourth panel of Figure 13 and are roughly a factor of 3 larger than those estimated by Gullbring et al. (1998).

Finally, the accretion luminosities are converted to mass accretion rates (Fig. 13, bottom panel) by assuming that the accretion shock luminosity equals the gravitational energy released per second as material free-falls along magnetic field lines from an inner disk radius of $3 R_{\text {star }}$ (e.g., Gullbring et al. 1998; Hartigan \& Kenyon 2003). This assumption will underestimate the accretion rate if a nonnegligible fraction of the gravitational energy is used to power a wind or jet (the accretion rate will increase by a factor of 2 in the case where $\frac{1}{2}$ the energy is used in mass-loss processes). Comparison of the mechanical luminosity of the outflow with the accretion luminosity suggests that this is unlikely ( $\S 3.3 .3)$. The stellar radii used in these calculations are determined from the temperatureradius relation of the Siess et al. (2000) $10^{6} \mathrm{yr}$ isochrone. Our geometric assumptions differ from those of Gullbring et al. (1998) only in the choice of inner disk radius. This small difference, in combination with the difference in accretion luminosities, leads to mass accretion rates that are, on average, a factor of 4 larger than those of Gullbring et al. (1998). The fifth panel of Figure 13 shows the distribution of inferred mass accretion rates.

The mass accretion rates for stars observed here are listed in Table 4 and span over 3 orders of magnitude, from $4 \times 10^{-10}$ to $7 \times 10^{-7} M_{\odot} \mathrm{yr}^{-1}$. The distribution of mass accretion rates determined for environmentally young stars and for the additional sample of class II stars is shown in Figure 14 as a function of the evolutionary diagnostics $T_{\text {bol }}$ and $\alpha$. The median mass accretion rate of class I stars is slightly less than that of class II stars (median class I $\log \dot{M}_{\text {acc }}=-8.1$; median class II $\log \dot{M}_{\text {acc }}=$ $-7.3)$. This is primarily because of the strong mass dependence of the accretion rate (e.g., White \& Basri 2003; Muzerolle et al. 2003b); unlike the class I sample, the class II sample does not extend into the substellar regime because of insufficient data for determining bolometric temperatures and spectral indices. Excluding the three class I brown dwarfs, the distributions of mass accretion rates for class I stars and class II stars are indistinguishable (K-S probability of 0.60$)$ and have more similar median values [median stellar class I $\left.\log \left(\dot{M}_{\text {acc }}\right)=-7.1\right]$. The distributions of mass accretion rates for $\mathrm{HH}$ stars and non- $\mathrm{HH}$ stars are also similar (K-S probability of 0.11 ), although $\mathrm{HH}$ stars have mass accretion rates that are larger by a factor of 2.5 in the median [median HH $\log \left(\dot{M}_{\text {acc }}\right)=-7.0$; median non-HH $\left.\log \left(\dot{M}_{\text {acc }}\right)=-7.4\right]$. Table 3 summarizes the median statistics, excluding brown dwarfs. Combining all types, the typical (median) mass accretion rate for an optically veiled K7-M1 star is $4 \times 10^{-8} M_{\odot} \mathrm{yr}^{-1}$, with a range of 2 orders of magnitude.
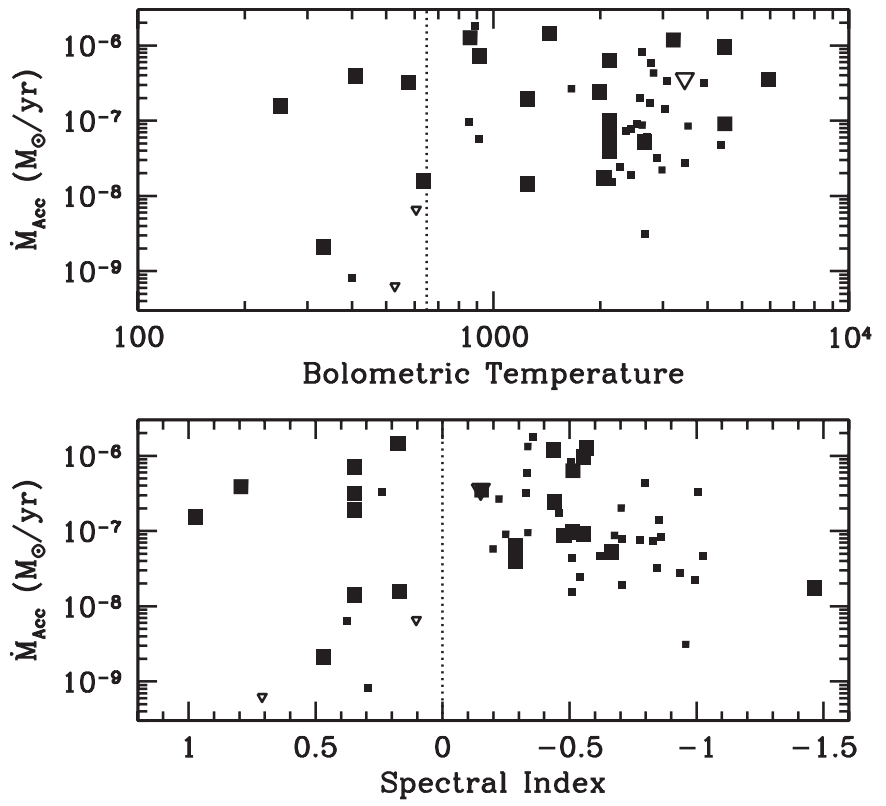

FIG. 14.-Mass accretion rate vs. bolometric temperature (top panel) and spectral index (bottom panel). The symbols are the same as in Fig. 4. Although the mass accretion rates for class I stars extend to lower values than for class II stars, this is primarily a consequence of lower masses. Over a similar stellar mass range, the mass accretion rates do not evolve significantly with either evolutionary diagnostic. HH stars have mass accretion rates that are similar to those of non-HH stars, although larger by a factor of $\sim 2.5$ on average.

The inconsistencies between the predictions of standard hot spot models and the large red excesses observed merit some discussion. Previous work has demonstrated that the variations in the veiling at blue wavelength are simultaneously correlated with variations at red wavelengths (see Fig. 4 in Basri \& Batalha 1990), suggesting a physically related origin. Emission from the inner disk is unlikely to contribute much optical excess flux if the temperature is limited to the dust destruction temperature of silicate-type grains $(\sim 1500 \mathrm{~K})$, as some observations suggest (Muzerolle et al. 2003a). The interpretation that we favor is that the this emission originates in a cooler component of the accretion-generated shock. If this cooler component dominates the excess at red wavelengths, then the observed constant flux over the wavelength range $6500-8400 \AA$ (Fig. 5) requires a surprisingly cool temperature of only $\sim 4000 \mathrm{~K}$. Thus, for a K7 star of similar temperature, the excess emission should be constant with wavelength, as observed (Fig. 5; Basri \& Batalha 1990). This cooler component must be featureless, suggesting that it is heated from above. The cooler excess component must also cover a large fraction of the star in order to be observed. For example, in the case in which the star and the excess have the same effective temperature (e.g., for a K7 star), the veiling, or the ratio of excess flux to photospheric flux, will be equal the ratio of the area of these emitting regions $\left(r=F_{\mathrm{ex}} / F_{\mathrm{phot}}=\right.$ $\left.A_{\text {ex }} / A_{\text {phot }}\right)$. Thus, for $r=1.0$, the cool featureless emission will cover $\frac{1}{2}$ the stellar surface. A more detailed investigation of the accretion-generated excess will require broader wavelength coverage than what is presented here (e.g., Stempels \& Piskunov 2003) and is consequently beyond the scope of this study.

\subsubsection{Mass-Loss Rates}

Optically thin forbidden-line emission can be used to estimate the mass-loss rate from young accreting stars. Forbidden emission lines such as [O I] $\lambda 6300$ and [S II] $\lambda 6731$ in the spectra of accreting $\mathrm{T}$ Tauri stars nearly always show emission 
at the systemic velocity and often show a separate blueshifted peak or blueshifted asymmetry (corresponding to velocities of a few tens to a few hundred $\mathrm{km} \mathrm{s}^{-1}$; see Appenzeller et al. 1984; Mundt et al. 1987; Edwards et al. 1987; Cabrit et al. 1990; Hamann 1994; Hartigan et al. 1995). The blueshifted emission is attributed to a high-velocity wind or jet on the near side of the star; the presence of a circumstellar disk is believed to prevent direct observation of the redshifted component of the presumed bipolar flow. High spatial resolution imaging studies of young stars with strong forbidden-line emission have shown that the high- and low-velocity components likely originate in separate regions. The low-velocity emission is usually spatially coincident with the stellar position, while the high-velocity emission appears spatially extended, often in the form of a wellcollimated jet (e.g., Mundt et al. 1987; Lavalley et al. 1997; Bacciotti et al. 2000). The presence of a high-velocity component of course requires the flow to be directed nearly along our line of sight; several systems with edge-on disk orientation power optical jets but have no high-velocity forbidden-line emission, as expected (§ 3.1.4). Interpretation of the lowvelocity forbidden-line emission is still uncertain (see, e.g., Hartigan et al. 1995); it too may originate from outflowing material at slower velocities, possibly a disk wind (Kwan \& Tademaru 1995).

We estimate mass outflow rates using the prescription given by Hartigan et al. (1995) for [S II] $\lambda 6731$ emission. In our spectra, this forbidden emission line is typically the strongest and the least contaminated by telluric or stellar features. Since the [S II] emission is optically thin, the observed [S II] luminosity can be used to estimate the total mass of the emission region. Hartigan et al. (1995) predict the mass of the emitting region to be $1.43 \times 10^{-3}\left(L_{6731} / L_{\odot}\right) M_{\odot}$, where $L_{6731}$ is the [S II] $\lambda 6731$ luminosity in solar units. This mass estimate assumes that the electron density is in the high-density limit $\left(N_{e}>2 \times\right.$ $10^{4} \mathrm{~cm}^{-3}$; consistent with [S II] line ratios), that all the sulfur atoms are singly ionized, and a cosmic sulfur abundance. With a mass $M$ of the emitting region, the mass-loss rate can then be estimated from the flow speed $V$ and the length scale $L$ over which the emission is observed $\left(\dot{M}_{\text {out }}=M V / L\right)$.

The EW[S II]s are converted to fluxes by assuming that the underlying continuum flux level is that of the star in the $R_{c}$ passband, as predicted by the Siess et al. (2000) 1 Myr isochrone, multiplied by $\left(1+r_{6500}\right)$ to account for veiling. This is the same continuum assumption used to estimate the mass accretion rates. The majority of stars observed do not have well-separated high- and low-velocity forbidden-line emission, which would allow us to directly measure the velocity of the flow and the fraction of high-velocity flux relative to the total line flux. Therefore, for all sources we assume a flow speed of $150 \mathrm{~km} \mathrm{~s}^{-1}$ and a high-velocity flux equal to $40 \%$ of the total [S II] $\lambda 6731$ line flux. This flow speed is the same as that used by Hartigan et al. (1995) and is consistent with the deprojected velocities of propagating $\mathrm{HH}$ knots (e.g., Krist et al. 1999; Bacciotti et al. 1999). The adopted percentage of high-velocity flux is the mean percentage of high-velocity emission measured by Hartigan et al. (1995; compare their Tables 3 and 4). The scale length is assumed to be the slit width of our observations (1".15), projected to the distance of Taurus (140 pc). We note that the slit width is of similar size to the seeing disk. Thus, even if the jet was projected along the length of the slit, the characteristic length scale would still be appropriate, since the width of the aperture extraction is defined by the seeing-limited width of the continuum and is relatively unaffected by spatially extended emission lines.
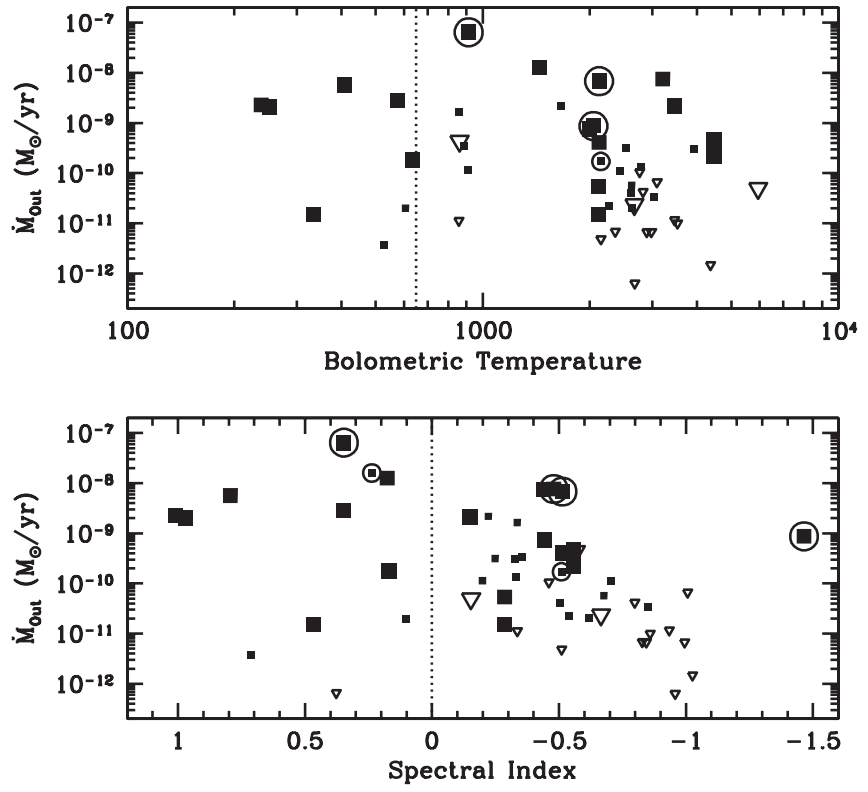

Fig. 15.-Mass outflow rate vs. bolometric temperature (top panel) and spectral index (bottom panel). The symbols are the same as in Fig. 4; circled symbols indicate stars with known or likely edge-on disk systems (see $\S 3.1 .4$ ). Over a similar stellar mass range, the mass outflow rates appears to decline by a median factor of $\sim 20$ with either bolometric temperature or spectral index. Similarly, HH stars have mass outflow rates that are, in the median, $\sim 20$ times that of non-HH stars. These difference may be a consequence of biases in the observed EW[S II] emission, as is the case for known edge-on disk systems.

Following this simple prescription, mass outflow rates are determined for all stars with [S II] $\lambda 6731$ emission, and upper limits to the mass outflow rate are determined in cases in which only detection limits are available. We note that for edge-on systems, the apparent bias toward larger forbidden emissionline EWs will correspondingly bias the inferred mass outflow rates. The determined mass outflow rates are listed in Table 4 and range from $2 \times 10^{-10}$ to $3 \times 10^{-6} M_{\odot} \mathrm{yr}^{-1}$. Ignoring edgeon systems, the largest mass outflow rate is then $6 \times 10^{-7} M_{\odot}$ $\mathrm{yr}^{-1}$ (DG Tau). The calculated mass outflow rates are shown in Figure 15 as a function of $T_{\text {bol }}$ and $\alpha$. Likely edge-on systems are indicated. [S II] $\lambda 6731$ measurements for the additional class II star sample are taken from Hartigan et al. (1995); the outflow rates are determined similarly. We note that the inferred measured mass outflow rates are slightly larger than, by a factor of 3 , the values determined from high spatial resolution spectroscopy (e.g., Bacciotti et al. 2002; Woitas et al. 2002). The mass outflow rates are also roughly a factor of 2 larger than those measured by Hartigan et al. (1995) using the highvelocity component of [O I] 26300 . The latter difference is primarily due to the slightly higher stellar luminosities of our adopted isochrone. Both samples show a very large dispersion in the mass outflow rate $(\sim 3$ orders of magnitude for K7-M1 spectral types). Thus, even though we use a weaker forbidden emission line to measure outflow rates, the similar dispersions suggest that the large range of values are dominated by stochastic differences between systems.

The median mass outflow rates of class I stars and class II stars are similar [median class I $\log \left(\dot{M}_{\text {out }}\right)=-8.0$; median class II $\left.\log \left(\dot{M}_{\text {out }}\right)=-8.2\right]$. Although the [S II] $\lambda 6731$ emission lines, from which the mass outflow rates are calculated, are systematically larger for class I stars than for class II stars, this dependence is less significant than the stellar mass dependence of the mass outflow rate. If the three class I brown dwarfs are 
excluded, the median mass outflow rate of class I stars is 31 times greater than that of class II stars [median stellar class I $\left.\log \left(\dot{M}_{\text {out }}\right)=-7.0\right]$. The distributions are nevertheless statistically indistinguishable (K-S probability of 0.29 ; edge-on disk systems have been excluded). The outflow rates of HH stars are, in general, greater than those of non-HH stars [median HH star $\log \left(\dot{M}_{\text {out }}\right)=-7.4$; median non-HH star $\log \left(\dot{M}_{\text {out }}\right)=$ $-8.7]$. These distributions show a more significant difference (K-S probability of 0.00049 ). Table 3 summarizes the median statistics, excluding brown dwarfs. Combining all types except edge-on systems, the typical (median) mass outflow rate for an optically veiled $\mathrm{K} 7-\mathrm{M} 1$ star is $2 \times 10^{-9} M_{\odot} \mathrm{yr}^{-1}$.

\subsubsection{Comparison of Mass Accretion and Mass-Loss Rates}

The measured mass outflow rates are correlated with the measured mass accretion rates over the 3 orders of magnitude in $\dot{M}_{\text {acc }}$ inferred here. This correlation has been demonstrated previously (e.g., Hartigan et al. 1995) and suggests a physically related origin, although it in part stems from the mass dependence of the accretion and outflow rates. This correlation is not uniquely one to one, however. There are 14 optically veiled stars that show no $[\mathrm{S}$ II] emission and three stars with optical veiling upper limits that show strong [S II] emission. Hartigan \& Kenyon (2003) note similar disparities using the stronger [O I] $\lambda 6300$ emission line.

Although both the inferred mass accretion and outflow rates depend directly on the photospheric luminosities, assumed in our analysis to correspond to $1 \mathrm{Myr}$ aged stars, the ratio of these rates is independent of the photospheric luminosity. These mass flow ratios are listed following the mass accretion and mass outflow rates in Table 5. In cases in which [S II] $\lambda 6731$ emission is measured but only veiling upper limits are available, the ratio is a lower limit. In cases in which the star is optically veiled but no [S II] $\lambda 6731$ emission is detected, the ratios are upper limits. These values are plotted versus the evolutionary diagnostics $T_{\mathrm{bol}}$ and $\alpha$ in Figure 16. The ratio $\dot{M}_{\text {out }} / \dot{M}_{\text {acc }}$ for five of the six edge-on disk systems is $\sim 10$. Since the majority of disk material is expected to accrete onto the star, as opposed to being ejected in an outflow, the large ratios for these systems support the hypothesis that the forbidden emission lines are biased toward artificially large values. Excluding edge-on disk systems, the measured ratios range from $3.2 \times$ $10^{-3}$ to 1.7 . The median log ratio (including limits) is -1.2 , corresponding to a ratio of 0.05 . This value is similar to, but larger by a factor of $\sim 5$ than, the average value measured by Hartigan et al. (1995).

Although the distributions of mass flow ratios for class I stars and class II stars span similar ranges, the mass flow ratios of class I stars are larger, in the median, than those of class II stars [median class I $\log \left(\dot{M}_{\text {out }} / \dot{M}_{\text {acc }}\right)=-0.04$; median class II $\left.\log \left(\dot{M}_{\text {out }} / \dot{M}_{\text {acc }}\right)=-1.2\right]$. The mass flow rates of HH stars are similarly larger than the values for non-HH stars [median HH star $\log \left(\dot{M}_{\text {out }} / \dot{M}_{\text {acc }}\right)=-0.13$; median non-HH star $\left.\log \left(\dot{M}_{\text {out }} / \dot{M}_{\text {acc }}\right)=-1.4\right]$. These differences are correlated, however, since many class I stars are also HH stars. Excluding class I stars, the median HH star $\log \left(\dot{M}_{\text {out }} / \dot{M}_{\text {acc }}\right)$ decreases to -0.81 . If many of the class I stars observed here have edge-on orientations ( $\S 4.5$ ), the effect of this on the observed forbidden-line EWs ( $(3.1 .4)$ could explain their higher ratios of $\dot{M}_{\text {out }} / \dot{M}_{\text {acc }}$.

The ratio of $\dot{M}_{\text {out }} / \dot{M}_{\text {acc }}$ appears to be independent of the mass accretion rate. Over the $\dot{M}_{\text {acc }}$ range $10^{-8}$ to $10^{-6} M_{\odot}$ $\mathrm{yr}^{-1}$, the mass flow ratios are uniformly distributed. We note
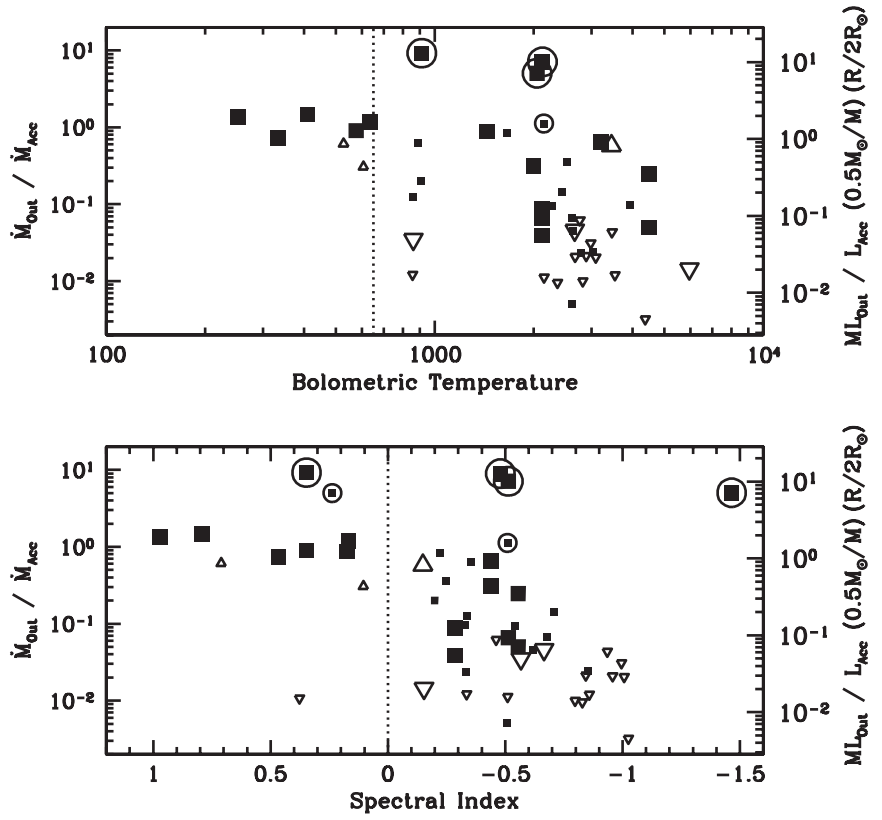

FIG. 16.-Ratio of mass outflow to mass accretion rate vs. bolometric temperature (top panel) and spectral index (bottom panel). The symbols are as in Fig. 4; upward pointing triangles are lower limits, and downward pointing triangles are upper limits. Circled symbols indicate stars with known or likely edge-on disk systems. The right-hand ordinate shows the corresponding ratio of outflow mechanical luminosity to accretion luminosity, assuming a $0.5 M_{\odot}$ star with a radius of $2.0 R_{\odot}$. Class I stars and $\mathrm{HH}$ stars have systematically larger ratios than class II stars or non-HH stars. These difference may be a consequence of biases in the observed EW[S II] emission, as is the case for known edge-on disk systems.

that the large range in $\dot{M}_{\text {out }} / \dot{M}_{\text {acc }}$ is not because of observational error but a consequence of significant dispersion in the relative strengths of the forbidden emission lines and the continuum excesses. The two observations of IRAS $04303+2240$, which measure $\log \left(\dot{M}_{\text {out }} / \dot{M}_{\text {acc }}\right)$ at -2.0 and -0.5 , suggest that this ratio changes for a given star-disk system (thereby excluding random orientation effects). These changes may arise either from actual changes in the mass accretion and/or mass outflow rates or from changes in the properties of the emission regions (e.g., temperature and density). The highly variable mass accretion rate will cause an additional source of variation for any given epoch. The previously ejected, spatially extended forbidden emission should be more correlated with a timeaveraged mass accretion rate, as opposed to the instantaneous measured mass accretion rates we present here.

Theoretical estimates for the ratio of mass outflow to mass accretion rate range from $\sim \frac{1}{3}$ (e.g., Shu et al. 1994) for a collimated jet powered by a magnetospheric accretion flow to $\sim 10^{-4}$ for a slow disk wind from a magnetically threaded accretion disk (e.g., Wardle \& Königl 1993). Although the highvelocity components and the current ranges of $\log \left(\dot{M}_{\text {out }} / \dot{M}_{\text {acc }}\right)$ appear to exclude low-velocity disk winds as a dominant massloss mechanism, the large range in mass flow ratios currently offer little constraint on proposed models for powering the observed jets.

Finally, the ratio $\dot{M}_{\text {out }} / \dot{M}_{\text {acc }}$ offers a useful measure of the mechanical luminosity of the outflow $\left(1 / 2 \dot{M}_{\text {out }} V^{2}\right)$ relative to the energy generated by accretion $\left(L_{\text {acc }}\right)$. The right-hand ordinate of Figure 16 shows this ratio, assuming accretion onto a $0.5 M_{\odot}$ star of radius $2.0 R_{\odot}$, and a mass-loss velocity $(V)$ of $150 \mathrm{~km} \mathrm{~s}^{-1}$. The median luminosity ratio is 0.076 . Thus, the 
mechanical luminosity of the outflow is approximately $8 \%$ of the observed accretion luminosity.

\section{DISCUSSION}

The insights gained from our spectroscopic observations address various long-standing assumptions regarding the earliest optically visible stages of star formation. In order to place our new results in context, we present a brief review of the standard paradigm of low-mass star formation and the taxonomy of young stars.

The formation of a low-mass star $\left(M \lesssim 3 M_{\odot}\right)$ is thought to begin with the dynamical inside-out collapse of a dense molecular cloud core (e.g., Shu et al. 1987). A three-stage description of evolution from this point toward the main sequence was proposed to explain three relatively distinct types of young stars as distinguished by their optical through millimeter SEDs (e.g., Lada 1987; Adams et al. 1987; Wilking et al. 1989). Young stars with SEDs that peak at far-infrared wavelengths $(30-100 \mu \mathrm{m})$ are presumed to be in the initial main accretion phase. These stars are often called class I stars or protostars. The majority of their luminosity escapes at far-infrared wavelengths as reprocessed radiation in the form of thermal dust emission; extinction to the star is often high. Young stars with SEDs that peak at near-infrared wavelengths are class II stars or classical T Tauri stars. Their SEDs typically resemble a revealed stellar photosphere with significant infrared and millimeter excess emission attributed to thermal emission from a circumstellar disk. Young stars with SEDs that peak in the optical, with little or no evidence for an infrared excess, are called class III stars or weak-lined T Tauri stars. These stars are presumed to have dispersed the majority of their circumstellar disk material. Subsequent to this three-class classification scenario, André et al. (1993) proposed a new class of less-evolved objects, called class 0 stars. These are stars that are so deeply embedded that they are only observable at far-infrared and millimeter wavelengths. Distinction between class 0 and class I stars is often observationally ambiguous, however (e.g., Young et al. 2003); both are believed to be in the main accretion phase, although the envelope is presumed to be more mass than the star during the class 0 stage.

The association of strong outflow/jet signatures, such as $\mathrm{HH}$ flows, with many protostars suggests that this process plays an important role in clearing envelope material. Within the class 0/I/II/III classification scheme, $\mathrm{HH}$ flows first appear associated with class 0 stars (e.g., IRAS 04368+2557; Eiroa et al. 1994), are most common among class I stars, are associated with about $10 \%$ of class II stars (Gomez et al. 1997), and are not associated with any class III stars. HH stars are therefore expected to be, on average, younger than most class II or class III T Tauri stars.

Since this classification scheme describing the evolution of circumstellar material around solar-type stars was first proposed, a wealth of direct observational evidence has been obtained in support of it. However, a correlation with stellar age has never been established. In this section we use the stellar properties (mass, rotation, age), and circumstellar properties (accretion rate, outflow rate, disk orientation, disk mass) of the 11 class I stars and 43 class II stars studied here to address issues related to the evolution from the protostellar stage to the $\mathrm{T}$ Tauri stage. We use these same properties to search for differences between HH stars and non-HH stars.

\subsection{The Masses of Class I Stars}

If the inside-out collapse of a singular isothermal sphere proceeds under the control of slow ambipolar diffusion pro- cesses (e.g., Shu 1977; Shu et al. 1987), the resulting infall rates are set by the local isothermal sound speed and therefore should remain roughly constant in time throughout the cloud. One implication is that more massive stars require more time to form than less massive stars. Thus, assuming coeval star formation, these conditions would yield class I stars that are more massive, on average, than class II stars. However, recent observational, theoretical, and numerical work suggests that supersonic turbulent flows rather than static magnetic fields control star formation (see the review by Mac Low \& Klessen 2004), affecting both the infall rate and the formation timescale. As an example, numerical models of gravoturbulent fragmentation suggest that the initial infall rates may be time dependent (initially large), scale with the final stellar mass, and yield a main accretion timescale that is nearly independent of the final stellar mass (Schmeja \& Klessen 2004).

Our results are more consistent with the proposal that supersonic turbulent flows control star formation, rather than static magnetic fields. As shown in Figure 11, the distributions of stellar mass for class I and class II stars are statistically indistinguishable. Although the sample of class I stars with mass estimates determined here is small, there are three brown dwarfs, six subsolar mass stars $\left(0.2-0.9 M_{\odot}\right)$, and two supersolar mass stars. Previous work has determined the masses of two class I stars based on disk kinematics under the assumption of Keplerian rotation $\left(0.2-0.4 M_{\odot}\right.$ for IRAS $04381+2540$ and $0.35-0.7 M_{\odot}$ for IRAS 04365+2535; Brown \& Chandler 1999). This distribution of class I star masses agrees very well with the initial mass function measured from $\mathrm{T}$ Tauri stars in Taurus (Briceño et al. 2002). The implication is that the known population of optically revealed T Tauri stars is likely to be a good representation of the final initial mass function produced by the cloud. The current and presumably subsequent generations of class I stars are unlikely to skew the mass function.

The cool spectral types of three class I stars suggest that they are class I brown dwarfs (IRAS 04248+2612, IRAS 04158+ 2805 , and IRAS $04489+3042$ ). With the exception of the most massive of these three, IRAS $04248+2612$, the $1.3 \mathrm{~mm}$ continuum fluxes suggest circumstellar disk+envelope masses of only a few hundredths of a solar mass (Motte \& André 2001). Thus, even if the majority of circumstellar material were to accrete onto these brown dwarfs, both IRAS $04158+2805$ and IRAS $04489+$ 3042 would remain substellar. If all of the $0.25 M_{\odot}$ envelope accretes onto IRAS $04248+2612$, it will be a low-mass star. IRAS $04248+2612$ is also of interest, as it is the lowest mass object known to power a molecular outflow (Moriarty-Schieven et al. 1992) and an HH object (HH 31; Gomez et al. 1997). We suggest that the lowest mass class I stars/brown dwarfs are low mass because they formed from lower mass cloud cores and not because they are at an earlier evolutionary stage and have not yet accreted the majority of their final mass. The implication is that the formation process of substellar mass objects, down to $\sim 0.05 M_{\odot}$, is simply a scaled down version of that for solar mass stars. Brown dwarfs likely form via the dynamical collapse of a cloud core, experience an embedded class I phase of evolution, and are capable of powering both molecular and HH flows. The presence of circumstellar material and accretion argues against low-mass star/brown dwarf formation scenarios involving early ejection from a competitively accreting cluster (e.g., Reipurth \& Clarke 2001).

Finally, we note that without stellar temperature estimates, previous attempts to estimate the stellar masses of protostars have proceeded by assuming accretion-dominated luminosities; the stellar mass can be estimated by calculating the gravitational 
potential well required to liberate the observed luminosity. The low luminosities of class I stars in Taurus consequently lead to very low substellar masses (e.g., Haro 6-33= IRAS 04385+ 2550; Young et al. 2003 estimate $M=0.01 M_{\odot}$ ). These masses are generally inconsistent with the values determined more directly here (e.g., Table 4; for Haro 6-33, $M=0.46 M_{\odot}$ ). Mass estimates assuming accretion-dominated luminosities appear to be inappropriate for many Taurus protostars.

\subsection{The Ages of Class I Stars}

Unlike the self-embedded class I stars, the optically revealed nature of class II and class III stars has allowed more direct measurements of their stellar temperatures and luminosities for comparison with the predictions of pre-main-sequence evolutionary models. These comparisons imply $\mathrm{T}$ Tauri ages of a few times $10^{6} \mathrm{yr}$, with no statistically significant difference between the H-R diagram locations of class II and class III stars (Kenyon \& Hartmann 1995; White \& Ghez 2001; Briceño et al. 2002). Class II and III stars also have similar lithium abundances (e.g., Strom et al. 1989; Martín et al. 1994), supporting similar ages. Given that class I stars in Taurus are approximately one-tenth as numerous as T Tauri stars (both class II and class III stars combined), it has been postulated that the class I phase lasts for roughly $10 \%$ of the T Tauri phase, under the assumption of a constant star formation rate (e.g., Myers et al. 1987; Kenyon et al. 1990). The implied statistical age of class I stars is then a few times $10^{5} \mathrm{yr}$.

With stellar properties now determined for $42 \%$ of the class I stars in Taurus, their stellar ages can be assessed more directly. As illustrated in Figure 10, environmentally young stars have ages that scatter uniformly about those of class II and class III stars, independent of the selection criteria used to identify them ( $T_{\text {bol }}, \alpha, \mathrm{HH}$ properties). The mean stellar luminosities of class I stars, specifically, are also more consistent with those of optically revealed T Tauri stars than with the stellar birth line, although the difference is only significant above $1.0 \mathrm{M}_{\odot}$. Class I stars appear to be coeval with class II and class III stars.

\subsection{The Early Evolution of Angular Momentum}

The accretion of high angular momentum material from a rotating, collapsing protostellar envelope during the earliest stages of star formation is expected to produce very rapidly rotating stars (e.g., Durisen et al. 1989), with rotational velocities comparable to the breakup velocity $\left[v_{\text {br }}=(G M / R)^{1 / 2} \sim 300 \mathrm{~km}\right.$ $\mathrm{s}^{-1}$. Thus, it came as a surprise to discover that most $\mathrm{T}$ Tauri stars, at least in the Taurus star-forming region, are slowly rotating. Nearly all of these stars have rotational velocities less than one-tenth $v_{\text {br }}$ (e.g., Hartmann et al. 1986; Bouvier 1990; Bouvier et al. 1995). Proposed explanations for the slow rotation rates usually involve "magnetic braking," a mechanism in which the star is magnetically coupled to the accretion disk and transfers angular momentum to the more slowly rotating parts of the outer disk (Blandford \& Payne 1982; Pudritz \& Norman 1986), the inner disk (Königl 1991; Shu et al. 1994), or possibly a stellar wind (Kwan \& Tademaru 1988). After the disk dissipates, substantial angular momentum loss must still occur, likely through a magnetically coupled stellar wind, in order to explain the slow rotation of somewhat older stars such as those in the Pleiades and Hyades (Stauffer et al. 1997; Krishnamurthi et al. 1998).

Thus far, the rotational properties of stars during the main accretion phase have been difficult to ascertain. Highdispersion near-infrared spectra of stars in the $\rho$ Oph dark cloud have found that at least some environmentally young stars rotate moderately rapidly $\left(v \sin i=30-50 \mathrm{~km} \mathrm{~s}^{-1}\right.$; Greene \& Lada 1997, 2000), with tentative evidence that stars with large spectral indices rotate the fastest (Doppmann et al. 2003), although this is based on only three stars with spectral indices greater than 0.0 (i.e., class I-like). Here we have demonstrated that class I stars in Taurus are, as a group, slowly rotating, with a $v \sin i$ distribution indistinguishable from that of the more environmentally evolved class II stars (Fig. 4). None of the observed class I stars in Taurus rotate with $v \sin i>30 \mathrm{~km} \mathrm{~s}^{-1}$. Further, since the class I luminosities and hence radii are similar to those of the class II stars, there is no evidence for any change in angular momentum from the class I to the class II stages (e.g., Fig. 12). The youngest optically visible stars have already been slowed to well below $(<5 \%-15 \%)$ breakup velocity. Stars in Taurus are known to be systematically slower rotators than stars in Orion (Clarke \& Bouvier 2000; White \& Basri 2003). Similar differences in the cloud properties or possibly stellar ages (see below) may explain apparent differences in the rotation rates of Taurus class I stars and $\rho$ Oph class I stars, once confirmed with a larger $\rho$ Oph sample.

It has been proposed that $\mathrm{T}$ Tauri stars in Taurus rotate more slowly than stars in other star-forming regions (e.g., $\rho$ Oph, Orion) because Taurus stars are older (by a factor of $\sim 3$ ), having ages larger than the expected disk braking timescale (Hartmann et al. 2002b). In this scenario, the slow rotational velocities of class I stars therefore corroborate the suggestion in $\S 4.2$ that these stars are as old as T Tauri stars. We speculate that if these class I stars indeed represent the longest lived disk population of class II stars ( $\S 4.6)$, this already slowly rotating sample could produce the "slow rotators" observed at intermediate pre-main-sequence ages (see Bouvier et al. 1997).

\subsection{The "Luminosity Problem" of Class I Stars, Revisited}

The mass infall rate during the main accretion phase can be approximated by dividing the local Jeans mass by the free-fall timescale $\left(\dot{M}_{\text {Infall }} \sim M_{\mathrm{J}} / \tau_{\mathrm{ff}}=5.4 c_{s}^{3} / G\right.$; Schmeja \& Klessen 2004). For an isothermal sound speed, $c_{s}$, of $0.2 \mathrm{~km} \mathrm{~s}^{-1}$ characteristic of Taurus, this corresponds to $\dot{M}_{\text {Infall }}=1 \times 10^{-5} M_{\odot}$ $\mathrm{yr}^{-1}$. The effects of magnetic support can produce smaller infall rates $\left(2 \times 10^{-6} M_{\odot} \mathrm{yr}^{-1}\right.$; Shu 1977$)$, while the external compression in turbulent flows can produce larger infall rates (up to $\sim 10^{-4} M_{\odot} \mathrm{yr}^{-1}$, at least initially; Schmeja \& Klessen 2004). Thus, if class I stars are in the main accretion phase, they should have mass infall rates consistent with these predictions. In support of this, density profiles corresponding to infall rates of a few times $10^{-6} M_{\odot} \mathrm{yr}^{-1}$ in envelope-only models successfully fit the SEDs and scattered light images of many class I stars (Kenyon et al. 1993a, 1993b; Whitney et al. 1997).

As first pointed out by Kenyon et al. (1990), if the infalling envelope material is channeled onto the star via steady state disk accretion at this rate, the liberated accretion luminosity would be roughly 10 times that emitted from the photosphere. With accretion-dominated luminosities, class I stars would have total luminosities roughly 10 times those of similar mass $\mathrm{T}$ Tauri stars. Direct tests of this prediction have been inhibited, however, by the lack of measured stellar and accretion luminosities of class I stars. Nevertheless, previous studies have tried to assess the relative contribution of the accretion luminosity, under the assumption of similar stellar luminosities, by comparing the bolometric luminosities of class I stars and class II/III T Tauri stars. Bolometric luminosities are calculated by integrating the entire energy distribution of a star. These comparisons show no statistically significant difference between these classes. The discrepancy between the observed 
bolometric luminosities and the predicted accretion-dominated luminosities is often referred to as the "luminosity problem" for class I stars (Kenyon et al. 1990, 1994).

The spectroscopic measurements of 11 class I stars observed here allow a more direct measure of the relative stellar and accretion contributions to the bolometric luminosity. We first confirm that the stellar, accretion, and disk emission can account for the observed bolometric luminosity. The total luminosity, $L_{\text {tot }}$, is calculated as

$$
L_{\mathrm{tot}}=L_{\mathrm{star}}+L_{\mathrm{acc}, \mathrm{sh}}+L_{\mathrm{acc}, d}+L_{\mathrm{rep}, d},
$$

where $L_{\text {star }}$ is the stellar luminosity calculated in $\S 3.2 .1$ (Table 4), $L_{\text {acc, sh }}$ is the accretion luminosity calculated in $\S 3.3 .1\left[L_{\text {acc, sh }}=\right.$ $\left.G M M_{\text {star }}\left(1 / R_{\text {star }}-1 / R_{\text {in }}\right)\right], L_{\text {acc }, d}$ is the viscously generated luminosity of the disk, and $L_{\mathrm{rep}, d}$ is the reprocessed luminosity from the disk. The third and fourth terms are calculated assuming a geometrically thin, optically thick circumstellar disk with an inner radius $\left(R_{\text {in }}\right)$ of $3 R_{\text {star }}$ and infinite outer radius. The luminosity viscously generated in the disk equals the change in potential energy of material moving from infinity to the inner disk edge, and thus $L_{\text {acc, } d}=G \dot{M} M_{\text {star }}\left(1 / R_{\text {in }}-1 / R_{\infty}\right)$, which simplifies to $L_{\mathrm{acc}, d}=0.5 L_{\mathrm{acc}, \text { sh }}$. Hence, $L_{\mathrm{acc}, d}$ can be determined from the inferred $L_{\text {acc, sh }}$ values. The luminosity reprocessed by the disk originates from two sources, the central star and the bright accretion shock, and thus $L_{\mathrm{rep}, d}=0.25\left(L_{\mathrm{star}}+L_{\mathrm{acc}, \mathrm{sh}}\right) R_{\mathrm{star}} / R_{\mathrm{in}}$ (Adams \& Shu 1986). The total luminosity can then be simplified as

$$
L_{\text {tot }}=1.08 L_{\text {star }}+1.58 L_{\text {acc, sh }} .
$$

The total luminosities calculated following this prescription agree well with the bolometric luminosities for both class I stars and class II stars [median class I $\log \left(L_{\mathrm{tot}} / L_{\mathrm{bol}}\right)=-0.08$, $\sigma=0.58$; median class II $\left.\log \left(L_{\text {tot }} / L_{\text {bol }}\right)=0.19, \sigma=0.43\right]$. The agreement suggests that the dominant luminosity sources are accounted for; the large scatter suggests that the absolute values of the luminosity sources are very uncertain. For the class I stars, this agreement suggests that there is not significant contribution to the bolometric luminosity generated from an infalling envelope accreting directly onto the disk at large radii.

For both class I and class II stars, the contribution of disk accretion $\left(L_{\mathrm{acc}, \mathrm{sh}}+L_{\mathrm{acc}, d}\right)$ to the bolometric luminosity can range from a few percent to $50 \%$. However, in the typical (median) case, only $25 \%$ of the bolometric luminosity is generated through disk accretion; the majority of the bolometric luminosity originates from the star (see also Muzerolle et al. 1998). We note that the bolometric luminosities of the 11 class I stars studied here [median $\log \left(L_{\text {bol }} / L_{\odot}\right)=-0.36, \sigma=0.61$ ] are very similar to the bolometric luminosities of the remaining 15 class I stars in Taurus [median $\log \left(L_{\mathrm{bol}} / L_{\odot}\right)=-0.17, \sigma=$ 0.56 ]. Thus, if the unobserved class I stars have a similar distribution of stellar luminosities, then they cannot have disk accretion rates as high as predicted by envelope infall models either. These results strongly favor one proposed resolution to the luminosity problem-class I stars do not have accretiondominated luminosities. However, this resolution raises two additional questions: (1) Are known class I stars properly classified? (2) Are class I stars in the main accretion phase? We address these two issues in the next sections.

\subsection{The Effect of Orientation on Class Classification}

In the traditional classification scheme, class I stars are true protostars - stellar embryos surrounded by an infalling envelope. However, the observational criteria traditionally used to identify class I stars distinctly from class II stars depend on the disk/envelope orientation relative to the line of sight. Radiative transfer models of still-forming stars predict that edgeon systems will have optical, near- and mid-infrared characteristics similar to less evolved, more embedded stars (Kenyon et al. 1993a, 1993b; Yorke et al. 1993; Sonnhalter et al. 1995; Men'shchikov \& Henning 1997; Whitney et al. 2003). As examples, class II stars viewed edge-on will, in many cases, resemble class I stars, while class I stars viewed at pole-on orientations will have optical/infrared properties more characteristic of class II stars. The predictions for pole-on orientations are less clear, however, since the distribution and radiative properties of material from bipolar flows are not included in most models. We note that if class II stars with disk inclinations of $90^{\circ} \pm 5^{\circ}$ appear "edge-on," then $8.7 \%$ of all disk systems should appear edge-on, assuming random orientations $\left(17.4 \%\right.$ if $i=90^{\circ} \pm$ $10^{\circ}$ ). It is important to recognize how these orientation effects could have introduced selection biases into both the optically revealed class I stars studied here and the known population of class I stars in Taurus.

As discussed in $\S 3.1 .4$, the emission-line profiles offer an indirect constraint on the star/disk orientation. The general trend of narrow emission line profiles at the systemic velocity suggests a bias toward edge-on orientations for the 11 class I stars with measured photospheric features. Only three of these stars show high-velocity $\left(>60 \mathrm{~km} \mathrm{~s}^{-1}\right)$ forbidden-line emission, compared to more than half of class II stars (e.g., Hartigan et al. 1995). The $\mathrm{H} \alpha 10 \%$ widths of these class I stars are systematically less than those of class II stars (§ 3.1.4). Only one of the 11 class I stars (HL Tau) shows evidence of a strong blueshifted absorption superposed on the $\mathrm{H} \alpha$ emission profile. In comparison, roughly half of class II stars show this absorption feature (e.g., Alencar \& Basri 2000). These combined results imply little or no high-velocity material along our line of sight for most class I stars, as expected for an edge-on orientation. An orientation bias could also explain the unusually large forbidden emission-line EWs of most class I stars; the somewhat extended forbidden emission line region may be more directly observable in this case than the obscured stellar photosphere. Overall, the emission-line profiles of most optically revealed class I stars favor nearly edge-on orientations. One clear exception is HL Tau. Its high-velocity forbidden emission lines and broad $\mathrm{H} \alpha$ profile with superposed absorption favor a less edge-on orientation. This orientation may explain why this is one of the most optically bright environmentally young stars.

The evidence for edge-on orientations does not necessarily imply that the observed sample of class I stars are in fact class II stars. The presence of spatially extended envelope material, as determined from image morphology at infrared and millimeter wavelengths, offers a more direct constraint on the evolutionary class. On the basis of criteria put forth by Motte \& André (2001), only 58\% (15 of 26) of the class I stars listed in Table 1 are true protostars. The remaining $42 \%$ (11 stars) have envelope masses $\lesssim 0.1 M_{\odot}$ and are spatially unresolved at $1.3 \mathrm{~mm}$ wavelengths (referred to as "unresolved class I sources" in Motte \& André 2001, these stars are marked as class I' stars in Table 1). Motte \& André (2001) suggest that these stars are more likely transitional class I/II stars or highly reddened class II stars. The complementary near-infrared morphology survey by Park \& Kenyon (2002) supports the claim that these 11 stars are not bona fide class I 
stars. ${ }^{4}$ However, we note that the morphological criteria used in these studies do not account for the luminosity and mass of the central star. IRAS $04158+2805$ and IRAS $04489+$ 3042 may appear more evolved and pointlike because they are lower luminosity class I brown dwarfs with smaller disks and envelopes.

We conclude that as many as one-half of the class I sample listed in Table 1, and in particular eight of 11 class I stars studied here, could be misclassified class II stars. The emission-line profiles and image morphology suggest that in many cases this misclassification may have been caused by a nearly edge-on orientation. We nevertheless continue to refer to all of these as class I stars, since the orientations and the mass effects are generally not known.

\subsection{Are Class I Stars in the Main Accretion Phase?}

Although the absolute values of the mass accretion rates remain considerably uncertain, typical class I and class II stars of K7-M1 spectral type have $\dot{M}_{\text {acc }} \approx 4 \times 10^{-8} M_{\odot} \mathrm{yr}^{-1}$ (§ 3.3.1). There is no difference between the disk accretion rates of class I stars and class II stars. Muzerolle et al. (1998) found similar results based on $\mathrm{Br} \gamma$ luminosity measurements and assumed stellar properties. Although the new disk accretion rates we determine in $\S 3.3 .1$ are somewhat larger than previous estimates (e.g., Gullbring et al. 1998), they are still 2 orders of magnitude less than the envelope infall rates inferred from SED modeling (a few times $10^{-6} M_{\odot} \mathrm{yr}^{-1}$ ).

We explore possible explanations for reconciling this discrepancy. One possibility suggested by Kenyon et al. (1990) is that the infalling envelope material is not transferred to the star via disk accretion in a steady state fashion. The accreting envelope mass accumulates in the circumstellar disk until it becomes gravitationally unstable (e.g., Larson 1984), leading to a marked increase in the mass accretion rate. This process may be related to the FU Orionis outburst phenomenon, which is currently understood as rapid $\left(\sim 10^{-5} M_{\odot} \mathrm{yr}^{-1}\right)$ but short-lived ( $\sim 100 \mathrm{yr}$ ) accretion through a circumstellar disk onto a central star (Hartmann \& Kenyon 1987). At this rate of accretion, class I stars would only need to spend $5 \%-10 \%$ percent of their lifetime in the high-accretion state to achieve typical $\mathrm{T}$ Tauri masses within 1 Myr. The most luminous class I star in Taurus, L1551 IRS 5 (20.9 $L_{\odot}$; Kenyon \& Hartmann 1995), has been proposed to be in an FU Ori-like outburst state, although a scenario in which it is an embedded star of a few solar masses satisfies its observed properties equally well.

If the envelope material of class I stars is accumulating in their circumstellar disks, they are expected to have more massive disks than class II stars. One direct way to test this hypothesis is with $1.3 \mathrm{~mm}$ continuum observation. Since circumstellar dust is mostly optically thin at these wavelengths, the emission should trace the total mass in circumstellar dust. Figure 17 shows the distribution of $1.3 \mathrm{~mm}$ flux densities versus the evolutionary diagnostics $T_{\text {bol }}$ and $\alpha$ for all stars in Taurus with these values, including the larger sample of class I stars not spectroscopically studied here. The $1.3 \mathrm{~mm}$ measurements of class II stars are taken from Beckwith et al. (1990),

\footnotetext{
4 Except for the two bona fide class I stars with optically revealed companions (MHO 1/2 and GV Tau AB), which could bias $T_{\text {bol }}$ estimates toward hotter temperatures, all bona fide class I stars with $T_{\text {bol }}$ estimates (11 of 13) have $T_{\text {bol }}<350 \mathrm{~K}$. A $T_{\text {bol }}<350 \mathrm{~K}$ may be a better distinguishing value for class I stars. A comparison of the spectral indices suggests no similar correction; the spectral indices of bona fide and non-bona fide class I stars overlap significantly.
}
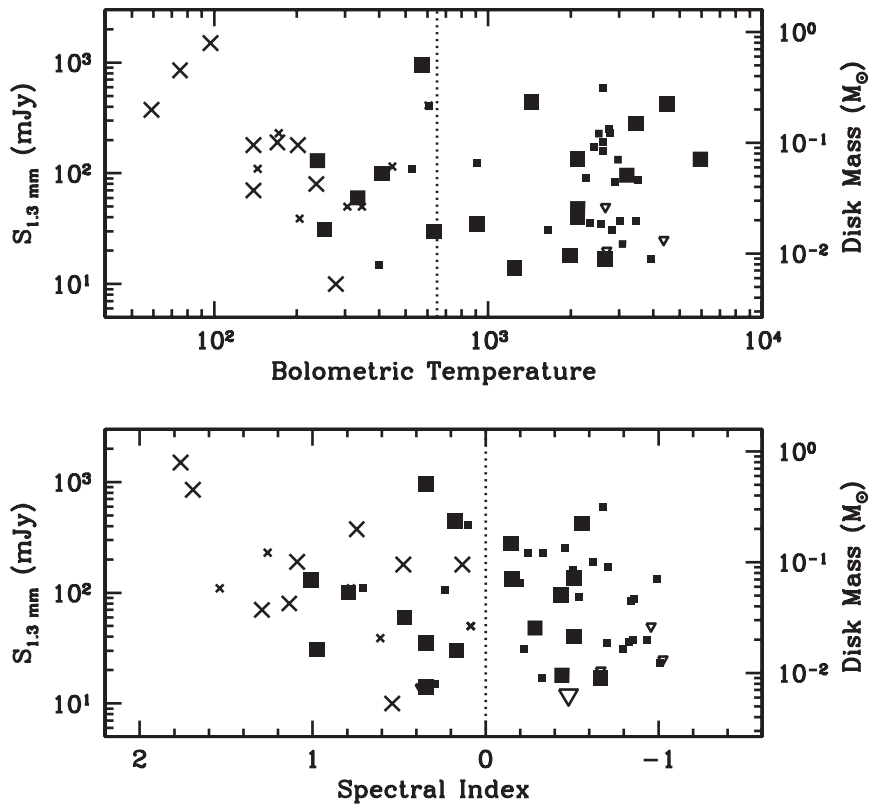

FIG. 17. - $1.3 \mathrm{~mm}$ flux density vs. bolometric temperature (top panel) and spectral index (bottom panel). The right-hand ordinate shows the conversion to disk mass, as described in the text $(\S 4.6)$. The symbols are as in Fig. 4 ; the crosses indicate class I stars without spectroscopically determined stellar properties. The disk mass does not evolve significantly with either evolutionary diagnostic. $\mathrm{HH}$ stars and non-HH stars have similar disk masses.

Osterloh \& Beckwith (1995), and Motte \& André (2001), which all have beam sizes of $\sim 11^{\prime \prime}-12^{\prime \prime}$. The emission in this beam is likely to be a good tracer of circumstellar disk mass without significant contamination from any possible envelope mass that would dominate on larger spatial scales (Motte \& André 2001). Figure 17 also indicates (right-hand ordinate) the corresponding disk mass for a dust opacity per unit mass column density of $0.01 \mathrm{~cm}^{2} \mathrm{~g}^{-1}$ and a dust temperature of $20 \mathrm{~K}$ for all sources. 5

As Figure 17 clearly demonstrates, the distributions of circumstellar disk masses for class I and class II stars are indistinguishable. On average, class I stars do not have more massive disks than class II stars. One possible caveat is that if much of the disk mass of class I stars is confined to be very close to the star ( $\lesssim 1 \mathrm{AU})$, as would be expected immediately prior to an FU Ori outburst, this mass would likely be optically thick and thus unrevealed in our $1.3 \mathrm{~mm}$ comparison. However, if the disks of class I stars undergo FU Ori outbursts at semiregular intervals ( $\sim 10 \%$ of the time), the optically thin outer disks would still need to be more massive than those of class II stars, on average, to sustain the larger time-averaged accretion rates. This is inconsistent with the observations and suggests that class I stars do not undergo FU Ori outbursts more often than class II stars.

An alternative possibility that we favor for reconciling the mass infall and disk accretion rates is that most of the class I stars in Taurus are not in the main accretion phase. First, the current populations of class I and class II stars are likely biased because the available low spatial resolution mid-infrared measurements (e.g., IRAS) used in class classification criteria (e.g., $\left.\alpha, T_{\text {bol }}\right)$ often include several young stars $(\S 2.1)$. Some

\footnotetext{
5 Although there is some evidence that both the dust opacity and temperature may change with evolutionary state, the expected changes are thought to be less than a factor of $\sim 2$ (Henning et al. 1995).
} 
class I and class II stars may have been misclassified as a consequence. In addition, as discussed in $\S 4.5,42 \%$ of stars classified as class I stars do not appear to be bona fide protostars (i.e., stars with spatially extended envelope structures). Many of these are likely to be class II or transitional class I/II stars that have been misclassified because of nearly edge-on disk orientations. Stars such as IRAS $04016+2610$ and IRAS $04302+2247$, as examples, have morphologies and kinematics that are better described by a rotating disklike structure (Hogerheijde \& Sandell 2000; Boogert et al. 2002; Wolf et al. 2003) than a collapsing envelope model (Kenyon et al. 1993b; Whitney et al. 1997). In the more general case, we suspect that the envelope infall rates have been overestimated, even for bona fide class I stars, by improperly accounting for the emission from a circumstellar disk. For an infalling envelope model, the ratio $\dot{M}_{\text {Infall }} / M_{\text {star }}^{1 / 2}$ sets the peak wavelength of the SED (Kenyon et al. 1993b); higher infall rates produce redder SEDs. However, the addition of an optically thick circumstellar disk also shifts the peak of the SED toward redder wavelengths (cf. Kenyon et al. 1993b; Wolf et al. 2003). Thus, if most class I stars have circumstellar disks as expected, then envelope-only models (i.e., no circumstellar disk) will systematically overestimate the mass infall rate in order to account for their redder SED.

We emphasize that even if, as we propose, most class I stars are not in the main accretion phase, they nevertheless must still have circumstellar envelopes. Disks alone are insufficient to explain the high extinctions, scattered light images, $1.3 \mathrm{~mm}$ morphologies, and mid- to far-infrared SEDs. Our proposal is simply that there is less mass in the envelope than has been presumed for many of the class I stars in Taurus. Consequently, the mass infall rates have been overestimated. Proper determination of the envelope mass and infall rate is especially challenging for the lowluminosity class I stars in Taurus, whose envelope emission can be confused with diffuse cloud emission (e.g., MHO 1/2, IRAS 04361+2547, IRAS 04381+2540; Motte \& André 2001). In addition, the large fraction of companion stars with $\sim 10^{3} \mathrm{AU}$ separations (Ducheñe et al. 2004) can give the impression of spatially extended structures. A convincing case for a massive infalling envelope can only be established by spatially mapping molecular line profiles and accounting for the effects of outflows and rotations (Evans 1999). Of the stars listed in Table 1, only the class 0 star IRAS $04368+2557$ (L1527) retains a massive extended envelope and shows unambiguous evidence for infall (Gregersen et al. 1997).

If class I stars are in fact as old as most T Tauri stars $(\sim 1 \mathrm{Myr}$; $\S 4.1)$, the presence of envelope material in these systems requires a long envelope dispersal timescale (i.e., comparable to $\mathrm{T}$ Tauri ages). A large dispersion in the disk dispersal timescale is thought to explain the apparently coeval populations of class II and class III stars. Extrapolating this idea to include high-latitude envelope material, many class I stars may represent a $\mathrm{T}$ Tauri star subsample with the longest envelope/disk dispersal timescale. The reason for this large dispersion is unclear but may involve variations in the initial properties of the cloud core, dynamical and radiative effects from the presence of close stellar companions, or the onset of planet formation.

The combined properties of class I stars in Taurus support a scenario in which most are not in the main accretion phase. For these systems, the stellar (or substellar) masses must have accumulated prior to the class I phase, during the class 0 phase. The flat density profiles of prestellar cores (Ward-Thompson et al. 1994) suggest initial conditions that favor more rapid accretion during the class 0 phase (Henriksen et al. 1997). The stronger mass outflows of class 0 stars relative to class I stars support this interpretation (Bontemps et al. 1996), if powered by mass accretion as suspected. A possible caveat to this suggestion is that if the class 0 phase is the main phase of accretion, the small number of known class 0 stars in Taurus suggests a very rapid formation timescale $\left(\sim 10^{4} \mathrm{yr}\right)$ and, consequently, a very high mass accretion rate $\left(\sim 10^{-4} M_{\odot} \mathrm{yr}^{-1}\right)$. These predictions are inconsistent with the relatively low luminosities of known class 0 stars (assuming they too are properly classified); class 0 stars would have their own luminosity problem. However, the $I R A S$ satellite may not have been sensitive enough to detect most class 0 stars in Taurus (e.g., IRAM 04191+1522; André et al. 1999). Indeed, early Spitzer Space Telescope results show that some "starless cores" may not be starless (Young et al. 2004). The 44 starless cores in Taurus (Onishi et al. 2002) may contain a yet hidden population of class 0 stars. Thus, concerns about the implied statistical ages and infall rates of class 0 stars in Taurus may be premature.

\subsection{Herbig-Haro Energy Sources}

Many of the class I and class II stars studied here have been identified as the energy sources of $\mathrm{HH}$ objects $(\S 2.1)$. Some studies suggest that $\mathrm{HH}$ stars have higher mass accretion rates and larger circumstellar reservoirs than the average T Tauri star (Reipurth et al. 1993; Chini et al. 1997). It has also been suggested that $\mathrm{HH}$ stars are more likely to be in binary systems (Reipurth 2000). The stellar and circumstellar properties inferred here may be used to identify possible differences between stars that power HH flows and those that do not.

The radial velocities of the $\mathrm{HH}$ stars newly measured here are consistent with the mean of Taurus, with the possible exception of HV Tau C $(\S 3.1 .1)$, which suggests that they are not more likely to be close (spectroscopic) binaries than non-HH stars. The locations of HH stars and non-HH stars on an H-R diagram are similar (Fig. 10); HH stars do not appear to be systematically younger. We do find, however, a decreasing frequency of HH objects associated with the lowest mass stars and brown dwarfs ( $\S \S 3.1 .2$ and 3.2.2). The frequency may be even less, as none of the recently identified accreting T Tauri brown dwarfs (e.g., Muzerolle et al. 2003b) are known to power HH flows. Although the strong forbidden line emission confirms that many low-mass stars/brown dwarfs indeed power jets, the decreased frequency of spatially resolved regions of shocked emission suggests that their jets may be less powerful. This result is consistent with the mass-dependent mass outflow rates and mechanical luminosities determined in $\S$ 3.3.2. If the velocity imparted to the outflow also decreases with stellar mass (we have assumed a constant value in our calculations), this would lead to an even stronger mass dependence on the mechanical energy of the outflow. Although the $v \sin i$ values of $\mathrm{HH}$ stars are somewhat larger than those of non-HH stars ( $§ 3.1 .1$ ), this difference disappears if the values are normalized by their breakup velocity $(\S 4.1)$. HH stars and non-HH stars have similar distributions of angular momentum.

In comparison with non-HH stars, HH stars have slightly higher mass accretion rates $(\times 2.5$ in the median) and much larger mass outflow rates ( $\times 20$ in the median). It is unclear whether the apparent difference in the mass outflow rate is a real time-averaged difference or a consequence of recent accretion/outflow history. The similar line profiles of most $\mathrm{HH}$ stars and non-HH stars (class I stars being the exception) suggest that edge-on orientation effects have not significantly biased the forbidden-line EWs of HH stars to large values (§ 3.1.4), which would translate into larger mass outflow rates. 
The distributions of millimeter fluxes, a direct tracer of circumstellar mass, for $\mathrm{HH}$ stars and non-HH stars are indistinguishable (Fig. 17). This result is in contrast to the study of Reipurth et al. (1993), who found that HH energy sources generally have, on average, more than 1 order of magnitude $1.3 \mathrm{~mm}$ wave flux densities than T Tauri stars in Taurus do. However, the Reipurth et al. (1993) comparison is biased, in that the majority of HH stars in that study are much more luminous $\left(1-10^{3} L_{\odot}\right)$ than the Taurus $\mathrm{T}$ Tauri stars with which they are compared $\left(\sim 1 L_{\odot}\right)$; as noted by Reipurth et al. (1993), the $1.3 \mathrm{~mm}$ flux shows a strong dependence on the bolometric luminosity. Our results suggest that when $\mathrm{HH}$ stars and non-HH stars of similar luminosities are compared, their millimeter fluxes are likewise similar. The lower luminosities of Taurus HH stars may stem from current identification criteria (spatially resolved optical jet; Reipurth 1999). Less energetic jets and lower luminosity sources are easier to identify in Taurus given its close proximity and low extinction. It is unclear whether the more luminous HH stars in other star-forming regions are at an earlier evolutionary stage or are simply more massive.

Finally, we note that since $\mathrm{HH}$ objects are more often associated with class I stars than class II stars, the visual extinction of $\mathrm{HH}$ stars is systematically larger than for non- $\mathrm{HH}$ stars. Overall, however, with the exception of apparently larger mass outflow rates, the stellar and circumstellar properties of $\mathrm{HH}$ stars and non-HH stars of similar mass are similar.

\section{SUMMARY}

Using the W. M. Keck I telescope, we obtained highdispersion $(R \sim 34,000)$ optical $(6330-8750 \AA)$ spectra of 15 class I stars and 21 class II stars in the nearby Taurus starforming region. Targets were selected on the basis of evidence for either an infrared-dominated luminosity as quantified by the SED diagnostics $T_{\text {bol }}$ and $\alpha$, or the presence of a spatially resolved optical jet $(\S 2.1)$. The optical emission from these environmentally young stars is spatially extended scattered light in some cases and faint pointlike in others. For 28 of these 36 stars, our measurements are the first high-dispersion optical spectra ever obtained. Photospheric features are detected in 11 of the class I stars ( $42 \%$ of known Taurus class I stars) and in all 21 of the class II stars; strong emission lines (e.g., $\mathrm{H} \alpha$ ) are detected in the spectra of all stars observed, even class I stars not visible on the POSS-II red plates. Complementary $I_{c}$-band images were obtained for the majority of class I stars observed spectroscopically.

Radial and rotational velocities are determined via crosscorrelation with rotationally broadened spectral standards. All stars have radial velocities that are consistent (within $3 \sigma$ ) with the mean of Taurus. No spectroscopic binaries are identified, although HV Tau C and HK Tau B are noted as possible single-lined spectroscopic binaries. All stars are slowly rotating $\left(v \sin i<35 \mathrm{~km} \mathrm{~s}^{-1}\right)$. Spectral types and continuum excesses at 6500 and $8400 \AA$ are determined from the best-fit rotationally broadened and veiled dwarf spectra. The inferred spectral types range from G8 to M6, with typical uncertainties of 1 spectral subclass. The new spectral types, in combination with $J, H, K_{s}$ photometry from the 2MASS database, are used to estimate visual extinctions and stellar luminosities. Masses and ages are determined from comparison with the Siess et al. (2000) evolutionary models. Surface gravity signatures in all spectra appear either dwarf-like or intermediate between those of dwarfs and giants, consistent with the modestly less than dwarf surface gravities expected from their H-R diagram positions. Emission features associated with pressure-sensitive lines inhibit direct determination of surface gravities from line profile analyses.

The measured continuum excesses range from 0.0 to 5.7 times that of the photosphere, with one source (IRAS 04303+ 2240 ) showing variations by a factor of $\sim 3$ between two observational epochs. For all stars, the continuum excesses appear to exhibit the trend of retaining constant flux over the 6500$8400 \AA$ wavelength interval. Although this has been observed previously (Basri \& Batalha 1990), current hot spot models (e.g., Kenyon et al. 1994; Calvet \& Gullbring 1998) cannot account for it. We interpret this emission as a cooler component of the shock, although emission from the inner disk cannot be completely excluded. Mass accretion rates are determined from the excess emission at $6500 \AA$ under the assumption of a magnetically channeled accretion flow. Mass accretion rates for K7-M1 spectral types span $\sim 2$ orders of magnitude, with a median value of $4 \times 10^{-8} M_{\odot} \mathrm{yr}^{-1}$. This median value is larger than previous estimates (e.g., Gullbring et al. 1998) determined from excess emission at shorter wavelengths $\lesssim 0.5 \mu \mathrm{m}$ and based on a model that does not account for the observed excesses at red optical wavelengths. Until the continuum excess spectrum can be more accurately modeled, this will contribute a factor of 3 systematic uncertainty in mass accretion rates.

$\mathrm{H} \alpha$ emission is detected from all sources, and in most cases a wealth of permitted (Fe II, Ca II) and forbidden ([O I], [N II], [S II]) emission lines are seen. On the basis of the relatively distinct emission-line profiles of three optically veiled (and presumably accreting) edge-on disk systems ( $\mathrm{HH} 30, \mathrm{HV}$ Tau, and HK Tau B), we suggest that CoKu Tau 1, IRAS 04260+ 2642, and ZZ Tau IRS also have nearly edge-on orientations. We confirm previous results that find larger forbidden-line emission associated with class I stars than class II stars. However, we attribute this to an orientation bias that allows a more direct view of the forbidden emission line region than the stellar photosphere, and not to larger mass outflow rates. Mass outflow rates are determined from the strength of [S II] $\lambda 6731$ emission, under the assumption of a bipolar jet. Excluding edge-on disk systems, which may bias the measured forbiddenline EWs to larger values, the mass outflow rates for K7-M1 spectral types span $\sim 3$ orders of magnitude, with a median value of $2 \times 10^{-9} M_{\odot} \mathrm{yr}^{-1}$. The ratios of $\dot{M}_{\text {outflow }} / \dot{M}_{\text {inflow }}$ span $\sim 2$ orders of magnitude, with a median value of 0.05 .

The inferred stellar and circumstellar properties are used to conduct statistical comparisons of class I and class II stars, as well as HH stars and non-HH stars. A summary of the median statistics is compiled in Table 5, with brown dwarfs excluded. The distribution of stellar masses of class I stars is similar to that of class II stars, ranging from substellar to several solar masses. Of particular interest are IRAS $04158+2805$, IRAS $04248+$ 2612, and IRAS 04489+3042, which have substellar masses. These are the first spectroscopically confirmed class I brown dwarfs. Brown dwarfs as low in mass as $0.05 M_{\odot}$ likely form via dynamical collapse of a cloud core, experience an embedded class I phase of evolution, and are capable of powering molecular and $\mathrm{HH}$ flows. Stellar luminosities of optically revealed class I stars suggest ages of $\sim 10^{6} \mathrm{yr}$, consistent with the ages of class II and class III T Tauri stars but inconsistent with ages implied by stellar birth line predictions and relative number statistics $\left(\sim 10^{5} \mathrm{yr}\right)$. In light of the wide range of disk dispersal timescales among $\mathrm{T}$ Tauri stars, we speculate that many class I stars represent a T Tauri subsample with the longest envelope/ disk dispersal timescale.

For both class I and class II stars, approximately $25 \%$ of the bolometric luminosity is generated through disk accretion. 
The majority of the bolometric luminosity originates from the star. This result strongly supports one proposed resolution of the "luminosity problem" for class I stars - they do not have accretion-dominated luminosities. We further propose that most class I stars in Taurus are past their main accretion phase. In some cases, class I stars may actually be class II stars that have been misclassified because of nearly edge-on orientations or because of a biased SED caused by spatially unresolved $25 \mu \mathrm{m}$ measurements. In the more general case, however, we suggest that the envelope infall rates, which are roughly 2 orders of magnitude larger than the observed disk accretion rates, have been overestimated by not properly accounting for the emission from a circumstellar disk.

While the proposed scenario for class I stars would explain the similar stellar properties (masses, ages, and rotation rates) and circumstellar properties (mass accretion rates and mass outflow rates) of class I and class II stars, there are still several issues that challenge this interpretation. The positions of class I stars are correlated with the positions of dense cores (Hartmann et al. 2002a), suggesting that they are still close to their birth site. It is somewhat surprising that they are not more dispersed, like class II stars, if class I stars are indeed as old as class II stars. The statistical ages of the few known class 0 stars suggest a very rapid formation timescale and large mass accretion rates; many class 0 stars will have an even more severe luminosity problem (e.g., IRAS 04368+2557). Moreover, their rarity implies that there is very little ongoing star formation in Taurus. We emphasize, however, that the limited sensitivity of current surveys makes it difficult to assess the validity of these socalled statistical problems. Observations with the Spitzer Space Telescope will likely yield a more comprehensive picture of star formation in the Taurus molecular cloud.

Many of the class I and class II stars studied here have been identified as the energy sources of $\mathrm{HH}$ objects. The primary difference between $\mathrm{HH}$ stars and non-HH stars is that $\mathrm{HH}$ stars have stronger forbidden-line emission, which translates into larger mass outflow rates by a factor of $\sim 20$ in the median. Whether this is a real time-averaged difference or a consequence of recent accretion/outflow history is unclear. We also find that HH flows are less commonly associated with very low mass stars $\left(\leqslant 0.2 M_{\odot}\right)$ and brown dwarfs than with more massive stars. One remarkable exception is the class I brown dwarf IRAS $04248+2612$, which powers both an $\mathrm{HH}$ and a molecular flow. Overall, with the exception of larger mass outflow rates, the stellar and circumstellar properties of $\mathrm{HH}$ stars and non-HH stars of similar mass are generally indistinguishable.

On a concluding and reflective note, as we anticipate the exciting young star discoveries likely to be provided by the sensitive Spitzer Space Telescope, it is perhaps fitting that we are now determining fundamental stellar and circumstellar properties of environmentally young stars identified $20 \mathrm{yr}$ ago by Spitzer's predecessor, the IRAS satellite. The extensive surveys of star-forming regions being conducted with Spitzer may revolutionize our generally accepted ideas regarding star formation in the same way that the enlightening discoveries of the IRAS satellite did. However, proper interpretation of newly discovered red, faint, and potentially very young objects will likely depend critically on understanding the relation between the stellar, accretion, and outflow properties, often best studied shortward of $\sim 1 \mu \mathrm{m}$, and the disk+envelope properties Spitzer will study at longer wavelengths. Observations of stars in the Taurus star-forming regions, with its close proximity and the low cloud extinction, will likely continue to play an important role in this endeavor.

We are grateful to G. Doppmann, S. Edwards, J. Eisner, P. Hartigan, L. Hartmann, M. Liu, and P. Williams for helpful discussions. This publication makes use of data products from the Two Micron All Sky Survey, which is a joint project of the University of Massachusetts and the Infrared Processing and Analysis Center/California Institute of Technology, funded by the National Aeronautics and Space Administration and the National Science Foundation. Finally, we recognize and acknowledge the very significant cultural role and reverence that the summit of Mauna Kea has always had within the indigenous Hawaiian community. We are most fortunate to have the opportunity to conduct observations from this mountain.
Adams, F. C., Lada, C. J., \& Shu, F. H. 1987, ApJ, 312, 788

Adams, F. C., \& Shu, F. H. 1986, ApJ, 308, 836

Alencar, S. H. P., \& Basri, G. 2000, AJ, 119, 1881

Appenzeller, I., Oestreicher, R., \& Jankovics, I. 1984, A\&A, 141, 108

Andre, P., Motte, F., \& Bacmann, A. 1999, ApJ, 513, L57

Andre, P., Ward-Thompson, D., \& Barsony, M. 1993, ApJ, 406, 122

Bacciotti, F., Eislöffel, J., \& Ray, T. P. 1999, A\&A, 350, 917

Bacciotti, F., Mundt, R., Ray, T. P., Eislöffel, J., Solf, J., \& Camezind, M. 2000, ApJ, 537, L49

Bacciotti, F., Ray, T. P., Mundt, R., Eislöffel, J., \& Solf, J. 2002, ApJ, 576, 222

Baraffe, I., Chabrier, G., Allard, F., \& Hauschildt, P. H. 1998, A\&A, 337, 403

Basri, G., \& Batalha, C. 1990, ApJ, 363, 654

Beckwith, S. V. W., Sargent, A. I., Chini, R. S., \& Güsten, R. 1990, AJ, 99, 924

Blandford, R. D., \& Payne, D. G. 1982, MNRAS, 199, 883

Bontemps, S., Andre, P., Terebey, S., \& Cabrit, S. 1996, A\&A, 311, 858

Boogert, A. C. A., Hogerheijde, M. R., \& Blake, G. A. 2002, ApJ, 568, 761

Bouvier, J. 1990, AJ, 99, 946

Bouvier, J., Covino, E., Kovo, O., Martin, E. L., Matthews, J. M., Terranegra, L., \& Beck, S. C. 1995, A\&A, 299, 89

Bouvier, J., et al. 1997, A\&A, 318, 495

Briceño, C., Hartmann, L. W., Stauffer, J., \& Martín, E. 1998, AJ, 115, 2074

Briceño, C., Luhman, K. L., Hartmann, L., Stauffer, J. R., \& Kirkpatrick, J. D.

2002, ApJ, 580, 317

Brown, D. W., \& Chandler, C. J. 1999, MNRAS, 303, 855

Burrows, C. J., et al. 1996, ApJ, 473, 437
REFERENCES

Cabrit, S., Edwards, S., Strom, S. E., \& Strom, K. M. 1990, ApJ, 354, 687

Calvet, N., Basri, G., \& Kuhi, L. V 1984, ApJ, 277, 725

Calvet, N., \& Gullbring, E. 1998, ApJ, 509, 802

Casali, M. M., \& Eiroa, C. 1996, A\&A, 306, 427

Chen, H., Myers, P. C., Ladd, E. F., \& Wood, D. O. S. 1995, ApJ, 445, 377

Chini, R., Reipurth, B., Sievers, A., Ward-Thompson, D., Haslam, C. G. T.,

Kreysa, E., \& Lemke, R. 1997, A\&A, 325, 542

Clarke, C. J., \& Bouvier, J. 2000, MNRAS, 319, 457

Cohen, J. G., Persson, S. E., Elias, J. H., \& Frogel, J. A. 1981, ApJ, 249, 481

Cohen, M., Dopita, M. A., \& Schwartz, R. D. 1986, ApJ, 307, L21

Decampli, W. M. 1981, ApJ, 244, 124

Doppmann, G. W., Jaffe, D. T., \& White, R. J. 2003, AJ, 126, 3043

Ducheñe, G., Bouvier, J., Bontemps, S., André, P., \& Motte, F. 2004, A\&A, in press

Ducheñe, G., Ménard, F., Stapelfeldt, K., \& Duvert, G. 2003, A\&A, 400, 559

Durisen, R. H., Yang, S., Cassen, P., \& Stahler, S. W. 1989, ApJ, 345, 959

Edwards, S., Cabrit, S., Strom, S. E., Heyer, I., Strom, K. M., \& Anderson, E. 1987, ApJ, 321, 473

Eiroa, C., Miranda, L. F., Anglada, G., Estalella, R., \& Torrelles, J. M. 1994, A\&A, 283, 973

Evans, N. J., II. 1999, ARA\&A, 37, 311

Fletcher, A. B., \& Stahler, S. W. 1994, ApJ, 435, 313

Ghez, A. M., Neugebauer, G., \& Matthews, K. 1993, AJ, 106, 2005

Gomez, M., Whitney, B. A., \& Kenyon, S. J. 1997, AJ, 114, 1138

Gray, D. 1992, The Observation and Analysis of Stellar Photospheres (Cambridge: Cambridge Univ. Press) 
Greene, T. P., \& Lada, C. J. 1996, ApJ, 461, 345 1997, AJ, 114, 2157

2000, AJ, 120, 430

Gregersen, E. M., Evans, N. J., II, Zhou, S., \& Choi, M. 1997, ApJ, 484, 256 Gullbring, E., Calvet, N., Muzerolle, J., \& Hartmann, L. 2000, ApJ, 544, 927

Gullbring, E., Hartmann, L. W., Briceño, C., \& Calvet, N. 1998, ApJ, 492, 323

Hogerheijde, M. R., \& Sandell, G. 2000, ApJ, 534, 880

Hamann, F. 1994, ApJS, 93, 485

Hamann, F., \& Persson, S. E. 1992, ApJS, 82, 247

Hartigan, P., Edwards, S., \& Ghandour, L. 1995, ApJ, 452, 736

Hartigan, P., \& Kenyon, S. J. 2003, ApJ, 583, 334

Hartigan, P., Kenyon, S. J., Hartmann, L., Strom, S. E., Edwards, S., Wlety, A. D., \& Stauffer, J. 1991, ApJ, 382, 617

Hartmann, L. 2002a, ApJ, 566, L29

- 2002b, ApJ, 578, 914

Hartmann, L., Hewett, R., \& Calvet, N. 1994, ApJ, 426, 669

Hartmann, L., Hewett, R., Stahler, S., \& Mathieu, R. D. 1986, ApJ, 309, 275

Hartmann, L., \& Kenyon, S. J. 1987, ApJ, 312, 243

Hayes, D. S. 1985, in Calibration of Fundamental Stellar Quantities, ed. D. S. Hayes, L. E. Pasinetti, \& A. G. D. Philip (Dordrecht: Reidel), 225

Henning, Th., Michel, B., \& Stognienko, R. 1995, Planet. Space Sci., 43, 1333

Henriksen, R., André, P., \& Bontemps, S. 1997, A\&A, 323, 549

Hillenbrand, L. A., \& White, R. J. 2004, ApJ, 604, 741

Jayawardhana, R., Mohanty, S., \& Basri, G. 2003, ApJ, 592, 282

Johns-Krull, C., Valenti, J. A., \& Koresko, C. 1999, ApJ, 516, 900

Joy, A. H. 1945, ApJ, 102, 168 1949, ApJ, 110, 424

Kenyon, S. J., Brown, D. I., Tout, C. A., \& Berlind, P. 1998, AJ, 115, 2491

Kenyon, S. J., Calvet, N., \& Hartmann, L. 1993a, ApJ, 414, 676

Kenyon, S. J., \& Hartmann, L. 1995, ApJS, 101, 117

Kenyon, S. J., Hartmann, L. W., Strom, K. M., \& Strom, S. E. 1990, AJ, 99, 869

Kenyon, S. J., Whitney, B. A., Gomez, M., \& Hartmann, L. 1993b, ApJ, 414,773

Kenyon, S. J., et al. 1994, AJ, 107, 2153

Kirkpatrick, J. D., Henry, T. J., \& McCarthy, D. W., Jr. 1991, ApJS, 77, 417

Königl, A. 1991, ApJ, 370, L39

Koresko, C. D. 1998, ApJ, 507, L145

Krishnamurthi, A., et al. 1998, ApJ, 493, 914

Krist, J. E., et al. 1998, ApJ, 501, 841 1999, ApJ, 515, L35

Kwan, J., \& Tademaru, E. 1988, ApJ, 332, L41 1995, ApJ, 454, 382

Lada, C. J. 1987, in IAU Symp. 115, Star Forming Regions, ed. M. Peimbert \& J. Jugaku (Dordrecht: Kluwer), 1

Landolt, A. U. 1992, AJ, 104, 340

Larson, R. B. 1984, MNRAS, 206, 197

Lavalley, C., Cabrit, S., Dougados, C., Ferruit, P., \& Bacon, R. 1997, A\&A, 327,671

Leinert, Ch., Zinnecker, H., Weitzel, N., Christou, J., Ridgway, S. T., Jameson, R., Haas, M., \& Lenzen, R. 1993, A\&A, 278, 129

Luhman, K. L., Briceño, C., Stauffer, J. R., Hartmann, L., Barrado y Navascués, D., \& Caldwell, N. 2003, ApJ, 590, 348

Lynden-Bell, D., \& Pringle, J. E. 1974, MNRAS, 168, 603

Mac Low, M., \& Klessen, R. S. 2004, Rev. Mod. Phys., 76, 125

Magazzú, A., \& Martín, E. L. 1994, A\&A, 287, 571

Martín, E. L., Dougados, C., Magnier, E., Ménard, F., Magazzú, A., Cuillandre, J.-C., \& Delfosse, X. 2001, ApJ, 561, L195

Martin, E. L., Rebolo, R., Magazzu, A., \& Pavlenko, Ya. V. 1994, A\&A, 282,503

Men'shchikov, A. B., \& Henning, T. 1997, A\&A, 318, 879

Mohanty, S., Basri, G., Jayawardhana, R., Allard, F., Hauschildt, P., \& Ardila, D. 2004, ApJ, 609, 854

Moriarty-Schieven, G. H., Wannier, P. G., Tamura, M., \& Keene, J. 1992, ApJ, 400, 260

Motte, F., \& André, P. 2001, A\&A, 365, 440

Mundt, R., Brugel, W. E., \& Bührke, T. 1987, ApJ, 319, 275
Mundt, R., \& Fried, J. W. 1983, ApJ, 274, L83

Mundt, R., Stocke, J., Strom, E. S., Strom, K. M., \& Anderson, E. R. 1985, ApJ, 297, L41

Muzerolle, J., Calvet, N., Hartmann, L., \& D’Alessio, P. 2003a, ApJ, 597, L149 Muzerolle, J., Hartmann, L., \& Calvet, N. 1998, AJ, 116, 455

Muzerolle, J., Hillenbrand, L., Calvet, N., Briceño, C., \& Hartmann, L. 2003b, ApJ, 592, 266

Myers, P. C., Fuller, G. A., Mathieu, R. D., Beichman, C. A., Benson, P. J., Schild, R. E., \& Emerson, J. P. 1987, ApJ, 319, 340

Myers, P. C., \& Ladd, E. F. 1993, ApJ, 413, L47

Nidever, D. L., Marcy, G. W., Butler, R. P., Fischer, D. A., \& Vogt, S. S. 2002, ApJS, 141, 503

Oke, J. B., et al. 1995, PASP, 107, 375

Onishi, T., Mizuno, A., Kawamura, A., Tachihara, K., \& Fukui, Y. 2002, ApJ, 575,950

Osterloh, M., \& Beckwith, S. V. W. 1995, ApJ, 439, 288

Padgett, D. L., Brandner, W., Stapelfeldt, K. R., Strom, S. E., Terebey, S., \& Koerner, D. 1999, AJ, 117, 1490

Park, S., \& Kenyon, S. J. 2002, AJ, 123, 3370

Press, W. H., Teukolsky, S. A., Vetterling, W. T., \& Flannery, B. P. 1997, Numerical Recipes in C (Cambridge: Cambridge Univ. Press)

Pudritz, R. E., \& Norman, C. A. 1986, ApJ, 301, 571

Reipurth, B. 1999, A General Catalogue of Herbig-Haro Objects, 2nd ed., http://casa.colorado.edu/hhcat/ 2000, AJ, 120, 3177

Reipurth, B., \& Bally, J. 2001, ARA\&A, 39, 403

Reipurth, B., Chini, R., Krugel, E., Kreysa, E., \& Sievers, A. 1993, A\&A, 273, 221

Reipurth, B., \& Clarke, C. 2001, AJ, 122, 432

Schmeja, S., \& Klessen, R. S. 2004, A\&A, 419, 405

Shu, F. H. 1977, ApJ, 214, 488

Shu, F. H., Adams, F. C., \& Lizano, S. 1987, ARA\&A, 25, 23

Shu, F. H., Najita, J., Ostriker, E., Wilkin, F., Ruden, S., \& Lizano, S. 1994, ApJ, 429, 781

Siess, L., Dufour, E., \& Forestini, M. 2000, A\&A, 358, 593

Sonnhalter, C., Preibisch, T., \& Yorke, H. W. 1995, A\&A, 299, 545

Stahler, S. W. 1988, ApJ, 332, 804

Stapelfeldt, K. R., Krist, J. E., Menard, F., Bouvier, J., Padgett, D. L., \& Burrows, C. J. 1998, ApJ, 502, L65

Stapelfeldt, K. R., Ménard, F., Watson, A. M., Krist, J. E., Dougados, C., Padgett, D. L., \& Brandner, W. 2003, ApJ, 589, 410

Stapelfeldt, K. R., et al. 1995, ApJ, 449, 888

Stauffer, J. R., Balachandran, S. C., Krishnamurthi, A., Pinsonneault, M., Terndrup, D. M., \& Stern, R. A. 1997, ApJ, 475, 604

Stempels, H. C., \& Piskunov, N. 2003, A\&A, 408, 693

Stocke, J. T., Hartigan, P. M., Strom, S. E., Strom, K. M., Anderson, E. R., Hartmann, L. W., \& Kenyon, S. J. 1988, ApJS, 68, 229

Strom, K. M., Wilkin, F. P., Strom, S. E., \& Seaman, R. L. 1989, AJ, 98, 1444

Tinney, C. G., \& Reid, I. N. 1998, MNRAS, 301, 1031

Torres-Dodgen, A. V., \& Weaver, W. B. 1993, PASP, 105, 693

Vogt, S. S., et al. 1994, Proc. SPIE, 2198, 362

Wardle, M., \& Königl, A. 1993, ApJ, 410, 218

Ward-Thompson, D., Scott, P. F., Hills, R. E., \& André, P. 1994, MNRAS, 268, 276

White, R. J., \& Basri, G. 2003, ApJ, 582, 1109

White, R. J., \& Ghez, A. M. 2001, ApJ, 556, 265

Whitney, B. A., Kenyon, S. J., \& Gomez, M. 1997, ApJ, 485, 703

Whitney, B. A., Wood, K., Bjorkman, J. E., \& Cohen, M. 2003, ApJ, 598, 1079

Wilking, B. A., Lada, C. J., \& Young, E. T. 1989, ApJ, 340, 823

Woitas, J., \& Leinert, Ch. 1998, A\&A, 338, 122

Woitas, J., Ray, T. P., Bacciotti, F., Davis, C. J., \& Eislöffel, J. 2002, ApJ, 580,336

Wolf, S., Padgett, D. L., \& Stapelfeldt, K. R. 2003, ApJ, 588, 373

Yorke, H. W., Bodenheimer, P., \& Laughlin, G. 1993, ApJ, 411, 274

Young, C. H., Shirley, Y. L., Evans, N. J., II, \& Rawlings, J. M. C. 2003, ApJS, 145,111

Young, C. H., et al. 2004, ApJS, 154, 396 